\title{
Enantioselective Synthesis of Indolizidines Bearing Quaternary Substituted Stereocenters via Rhodium-Catalyzed [2+2+2] Cycloaddition of Alkenyl Isocyanates and Terminal Alkynes
}

\author{
Ernest E. Lee and Tomislav Rovis* \\ Department of Chemistry, Colorado State University, Fort Collins, Colorado 80523. \\ E-mail: rovis@lamar.colostate.edu
}

\section{Supporting Information}

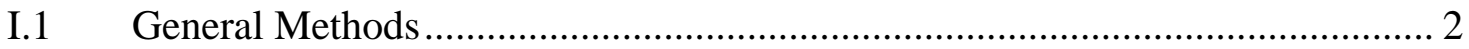

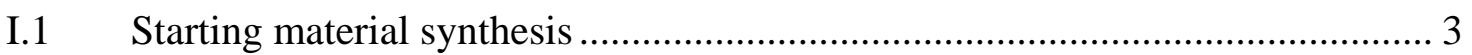

I.2 Procedures for alkenyl acid synthesis ................................................. 4

I.3 $\quad{ }^{1} \mathrm{H}$ and ${ }^{13} \mathrm{C}$ NMR Spectra for new carboxylic acids....................................... 6

I.4 General procedure for isocyanate synthesis.............................................. 13

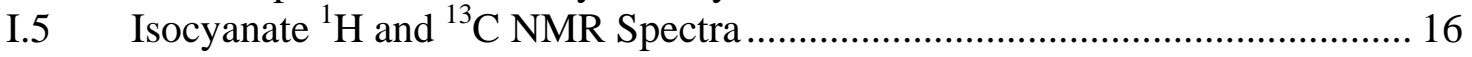

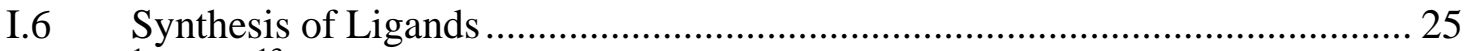

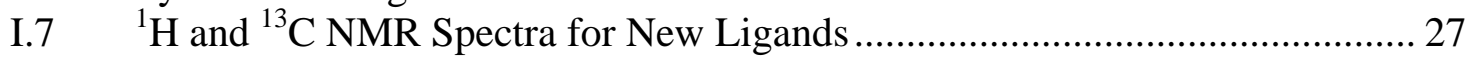

I.8 General procedure for [2+2+2] cycloadditions with Aryl Alkynes................. 30

I.9 $\quad{ }^{1} \mathrm{H}$ and ${ }^{13} \mathrm{C}$ NMR Spectra for [2+2+2] Products from Aryl Alkynes................. 35

I.10 General procedure for [2+2+2] cycloadditions with Alkyl Alkynes. .............. 48

I.11 ${ }^{1} \mathrm{H}$ and ${ }^{13} \mathrm{C}$ NMR Spectra for [2+2+2] Products from Alkyl Alkynes .............. 52

I.12 Characterization of Pyridone Side Products. ................................................. 61

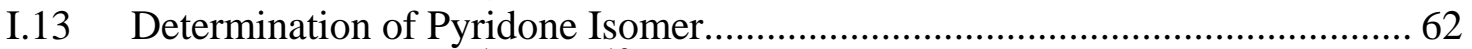

I.14 Pyridone Byproduct ${ }^{1} \mathrm{H}$ and ${ }^{13} \mathrm{C}$ NMR Spectra........................................... 63 


\section{I.1 General Methods}

Toluene, tetrahydrofuran, ether, and dichloromethane were degassed with argon and passed through one column of neutral alumina and one column of Q5 reactant. Triethylamine (peptide synthesis grade) was purchased from Fisher Scientific and used without further purification. Flash column chromatography was carried out on silica gel (60 A, 230 - 400 mesh, obtained from Silicycle Inc.) and was performed with reagent grade solvents. Analytical thinlayer chromatography (TLC) was performed on Silicycle glass-backed silica gel plates (60 $\AA$, $0.25 \mathrm{~mm}$, purchased from Silicycle Inc.), visualized with a UV lamp (254 nm) and/or potassium permanganate.

Infrared spectra (IR) were obtained on a Nicolet Avatar 320 FT-IR spectrometer. ${ }^{1} \mathrm{H}$ NMR and ${ }^{13} \mathrm{C}$ NMR were obtained on Varian Unity 300 and Unity 400 spectrometers. Chemical shifts are expressed in ppm values. Proton chemical shifts in $\mathrm{CDCl}_{3}$ were referenced to $7.26 \mathrm{ppm}$ $\left(\mathrm{CHCl}_{3}\right)$ or $0.00 \mathrm{ppm}$ (TMS). Carbon chemical shifts were referenced to $77.2 \mathrm{ppm}\left(\mathrm{CDCl}_{3}\right)$. Peak multiplicities are designated by the following abbreviations: s, singlet; d, doublet; t, triplet; q, quartet; m, multiplet; dd, doublet of doublets; dt, doublet of triplet; b, broad; $J$, coupling constant in Hz. Low resolution mass spectra (MS) and high resolution mass spectra (HRMS) were recorded on a Fisons VG Autospec spectrometer. HPLC spectra were obtained on an Angilent 1100 series system. Optical rotation was obtained with an Autopol-III automatic polarimeter. Melting points were obtained on a Fisher-Johns melting point apparatus and are uncorrected. References following the compound names indicate literature articles where the compound has been previously been reported.

Unless otherwise indicated, commercially available starting materials were purchased from Aldirch Chemicals. $\left[\mathrm{Rh}(\text { ethylene })_{2} \mathrm{Cl}\right]_{2}$ was purchased from Strem Chemicals. Commercially available ligand $\mathbf{L} 3$ was prepared from diethyl-tartrate according to literature procedures. 


\section{I.1 Starting material synthesis}<smiles>CC(=O)CCCC(=O)O</smiles>

5-Oxo-hexanoic acid: commercially available.<smiles>C=CCCC(=O)CCCC(=O)OCc1ccccc1</smiles>

5-Oxo-non-8-enoic acid and 5-Benzyloxymethyl-hex-5-enoic acid were prepared as described: Meyers, A. I.; Andres, C. J.; Resek, J. E.; Woodall, C. C.; McLaughlin, M. A.; Lee, P. H.; Price, D. A. Tetrahedron 1999, 55, 8931-8952.

\section{The remaining keto-acids were synthesized as follows:}

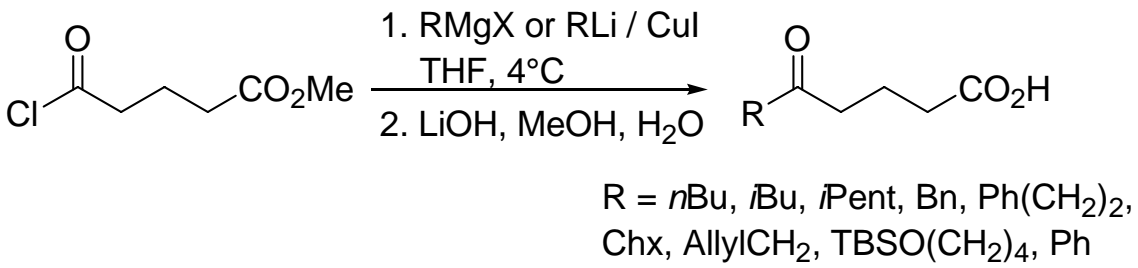

In a flame dried flask under Ar atmosphere, a solution of Grignard (1.0M in THF or $\mathrm{Et}_{2} \mathrm{O}, 14.5$ $\mathrm{mL}, 14.5 \mathrm{mmol}$ ) or $n$-BuLi (for $\mathrm{R}=n \mathrm{Bu}, 1.6 \mathrm{M}, 9.0 \mathrm{~mL}$ ) was slowly added to a stirring suspension of copper(I) iodide (1.38 g, $7.24 \mathrm{mmol})$ in THF $(30 \mathrm{~mL})$ at $4{ }^{\circ} \mathrm{C}$. After 30 minutes, methyl 5-chloro-5-oxo-valerate $(1.00 \mathrm{~mL}, 7.24 \mathrm{mmol})$ was added. The reaction was stirred at 4 ${ }^{\circ} \mathrm{C}$ for 3 hours and then quenched with $1 \mathrm{M} \mathrm{HCl}$, extracted $\left(\mathrm{Et}_{2} \mathrm{Ox} 3\right)$, dried over $\mathrm{MgSO}_{4}$, filtered and concentrated in vacuo. The keto-ester was purified by flash chromatography (Hexanes:EtOAc;93:7). The keto-ester (0.2 M) was then added to a stirring suspension of $\mathrm{LiOH}$ (5 eq) in $\mathrm{MeOH} / \mathrm{H}_{2} \mathrm{O}(3 / 1)$ and stirred at room temperature for 16 hours. The reaction was then quenched with $1 \mathrm{M} \mathrm{HCl}$, extracted $\left(\mathrm{Et}_{2} \mathrm{Ox} 3\right)$, dried over $\mathrm{MgSO}_{4}$, filtered and concentrated in vacuo. If suitably pure, the crude keto-acid was carried forward to the next step. If not suitably pure, the keto-acid was purified by silica gel flash chromatography (Hexanes:EtOAc4:1). 


\section{I.2 Procedures for alkenyl acid synthesis}

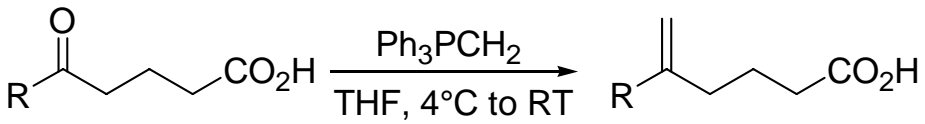

In a flame dried flask under Ar atmosphere, $n$-BuLi (1.6M in hexanes, $5.63 \mathrm{~mL}, 9.0 \mathrm{mmol})$ was added to a stirring solution of methyltriphenylphosphonium bromide ( $3.21 \mathrm{~g}, 9.0 \mathrm{mmol}$ ) in THF $(40 \mathrm{~mL})$ at $4{ }^{\circ} \mathrm{C}$. After stirring for 1 hour, ketoacid $(3.0 \mathrm{mmol})$ was added dropwise to the bright orange solution. The reaction was then warmed to room temperature and stirred overnight. The reaction was then quenched with $1 \mathrm{M} \mathrm{HCl}$, extracted $\left(\mathrm{Et}_{2} \mathrm{Ox} 3\right)$, dried over $\mathrm{MgSO}_{4}$, filtered and concentrated in vacuo. The crude material was then purified by silica gel flash chromatography.<smiles>C=C(C)CCCC(=O)O</smiles>

5-Methyl-hex-5-enoic acid: Flash Chromatography (Hexanes:EtOAc;4:1) yielded a clear liquid (67\%). $\mathrm{R}_{f}=0.32$ (Hexanes:EtOAc;3:1); IR (Thin Film) v 3076, 2939, 1709, 1414, 1292, 1239, $890 \mathrm{~cm}^{-1}$; ${ }^{1} \mathrm{H}$ NMR (400 MHz, CDCl $) \delta 4.72(1 \mathrm{H}, \mathrm{s}), 4.67(1 \mathrm{H}, \mathrm{s}), 2.33(2 \mathrm{H}$, t, $J=7.5 \mathrm{~Hz}), 2.04(2 \mathrm{H}, \mathrm{t}, J=7.5 \mathrm{~Hz}), 1.76(2 \mathrm{H}, \mathrm{tt}, J=7.5,7.5 \mathrm{~Hz}), 1.69(3 \mathrm{H}, \mathrm{s}) ;{ }^{13} \mathrm{C}$ NMR (100 MHz, $\left.\mathrm{CDCl}_{3}\right) \delta 180.6,144.7,111.0,37.1,33.6,22.6,22.4$.<smiles>C=C(CCC)CCCC(=O)O</smiles>

5-Methylene-nonanoic acid: Flash Chromatography (Hexanes:EtOAc;4:1) yielded a clear liquid (47\%). $\mathrm{R}_{f}=0.25$ (Hexanes:EtOAc;3:1); ${ }^{1} \mathrm{H}$ NMR (400 MHz, CDCl 3 ) $\delta 4.72$ (1H, s), 4.69 (1H, s), $2.33(2 \mathrm{H}, \mathrm{t}, J=7.0 \mathrm{~Hz}), 2.04(2 \mathrm{H}, \mathrm{t}, J=7.5 \mathrm{~Hz}), 1.98(2 \mathrm{H}, \mathrm{t}, J=7.5 \mathrm{~Hz}), 1.75(2 \mathrm{H}, \mathrm{tt}, J=7.5,7.5$ $\mathrm{Hz}), 1.41-1.22(4 \mathrm{H}, \mathrm{m}), 0.88(3 \mathrm{H}, \mathrm{t}, J=7.5 \mathrm{~Hz}) ;{ }^{13} \mathrm{C} \mathrm{NMR}\left(125 \mathrm{MHz}, \mathrm{CDCl}_{3}\right) \delta 180.5,148.9$, 109.7, 35.7, 35.4, 33.7, 30.1, 22.8, 22.7, 14.2.<smiles>C=C(CCCC(=O)O)CC(C)C</smiles>

7-Methyl-5-methylene-octanoic acid: Flash Chromatography (Hexanes:EtOAc;4:1) yielded a clear liquid (56\%). $\mathrm{R}_{f}=0.20$ (Hexanes:EtOAc;3:1); IR (Thin Film) v 2958, 1709, 1410, $1368 \mathrm{~cm}^{-}$ ${ }^{1} ;{ }^{1} \mathrm{H}$ NMR (400 MHz, CDCl 3 ) $\delta 4.73(1 \mathrm{H}, \mathrm{s}), 4.70(1 \mathrm{H}, \mathrm{s}), 2.34(2 \mathrm{H}, \mathrm{t}, J=7.0 \mathrm{~Hz}), 2.01(2 \mathrm{H}, \mathrm{t}, J$ $=7.5 \mathrm{~Hz}), 1.85(2 \mathrm{H}, \mathrm{d}, J=7.0 \mathrm{~Hz}), 1.79-1.67(3 \mathrm{H}, \mathrm{m}), 0.84(6 \mathrm{H}, \mathrm{d}, J=7.0 \mathrm{~Hz}) ;{ }^{13} \mathrm{C}$ NMR $(100$ $\left.\mathrm{MHz}, \mathrm{CDCl}_{3}\right) \delta 180.1,147.6,111.2,45.9,35.0,33.7,26.2,22.7,22.7$.<smiles>C=C(CCCC(=O)O)Cc1ccccc1</smiles>

5-Benzyl-hex-5-enoic acid: Flash Chromatography (Hexanes:EtOAc;4:1) yielded a clear liquid (60\%). $\mathrm{R}_{f}=0.35$ (Hexanes:EtOAc;4:1); IR (Thin Film) v 3027, 2934, 1708, 1495, 
1453, 1243, $896 \mathrm{~cm}^{-1} ;{ }^{1} \mathrm{H}$ NMR (400 MHz, $\left.\mathrm{CDCl}_{3}\right) \delta$ 7.31-7.26 (2H, m), 7.24-7.17 (3H, m), $4.86(1 \mathrm{H}, \mathrm{s}), 4.81(1 \mathrm{H}, \mathrm{s}), 3.34(2 \mathrm{H}, \mathrm{s}), 2.34(2 \mathrm{H}, \mathrm{t}, J=7.0 \mathrm{~Hz}), 2.03(2 \mathrm{H}, \mathrm{t}, J=7.5$ $\mathrm{Hz}), 1.79(2 \mathrm{H}, \mathrm{tt}, J=7.0,7.0 \mathrm{~Hz}) ;{ }^{13} \mathrm{C} \mathrm{NMR}\left(100 \mathrm{MHz}, \mathrm{CDCl}_{3}\right) \delta 180.5,147.9,139.7$, 129.2, 128.5, 126.4, 112.3, 43.0, 34.7, 33.7, 22.6.<smiles>C=C(CCCC(=O)O)C(C)C</smiles>

6-Methyl-5-methylene-heptanoic acid: Flash Chromatography (Hexanes:EtOAc;4:1) yielded a clear liquid (35\%). $\mathrm{R}_{f}=0.20$ (Hexanes:EtOAc;3:1); IR (Thin Film) v 2963, 1709, 1462, $1288 \mathrm{~cm}^{-}$ ${ }^{1} ;{ }^{1} \mathrm{H}$ NMR (400 MHz, CDCl $) \delta 4.76(1 \mathrm{H}, \mathrm{s}), 4.67(1 \mathrm{H}, \mathrm{s}), 2.35(2 \mathrm{H}, \mathrm{t}, J=7.5 \mathrm{~Hz}), 2.20(1 \mathrm{H}$, sext, $J=7.5 \mathrm{~Hz}), 2.06(2 \mathrm{H}, \mathrm{t}, J=7.5 \mathrm{~Hz}), 1.77(2 \mathrm{H}, \mathrm{tt}, J=7.0,7.0 \mathrm{~Hz}), 1.00(6 \mathrm{H}, \mathrm{d}, J=7.0 \mathrm{~Hz})$; ${ }^{13} \mathrm{C}$ NMR $\left(125 \mathrm{MHz}, \mathrm{CDCl}_{3}\right) \delta 180.6,154.8,107.3,33.8,33.7,23.1,21.9$.<smiles>C=C(CCCC(=O)O)C1CCCCC1</smiles>

5-Cyclohexyl-hex-5-enoic acid: Flash Chromatography (Hexanes:EtOAc;4:1) yielded a clear liquid (38\%). $\mathrm{R}_{f}=0.22$ (Hexanes:EtOAc;3:1); IR (Thin Film) $v$ 2926, 2852, 1709, 1640, 1449, $1262 \mathrm{~cm}^{-1}$; ${ }^{1} \mathrm{H}$ NMR (300 MHz, $\left.\mathrm{CDCl}_{3}\right) \delta 4.80(1 \mathrm{H}, \mathrm{s}), 4.75(1 \mathrm{H}, \mathrm{s}), 2.42$ (2H, t, $\left.J=7.5 \mathrm{~Hz}\right), 2.12$ $(2 \mathrm{H}, \mathrm{t}, J=7.5 \mathrm{~Hz}), 1.88-1.68(7 \mathrm{H}, \mathrm{m}), 1.34-1.14(5 \mathrm{H}, \mathrm{m}) ;{ }^{13} \mathrm{C} \mathrm{NMR}\left(100 \mathrm{MHz}, \mathrm{CDCl}_{3}\right) \delta 180.6$, $154.1,107.8,44.1,34.3,33.8,32.6,26.9,26.5,23.2$.

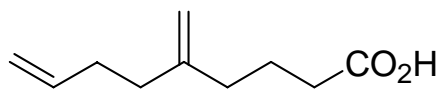

5-Methylene-non-8-enoic acid: Flash Chromatography (Hexanes:EtOAc;4:1) yielded a clear liquid (58\%). $\mathrm{R}_{f}=0.26$ (Hexanes:EtOAc;3:1); IR (Thin Film) v 2934, 1709, 1643, 1414, 1289 $\mathrm{cm}^{-1}$; ${ }^{1} \mathrm{H}$ NMR $\left(400 \mathrm{MHz}, \mathrm{CDCl}_{3}\right) \delta 5.79(1 \mathrm{H}, \mathrm{ddd}, J=17.0,10.0 \mathrm{~Hz}), 5.00(1 \mathrm{H}, \mathrm{dt}, J=17.0,1.0$ $\mathrm{Hz}), 4.93(1 \mathrm{H}, \mathrm{d}, J=10.0 \mathrm{~Hz}), 4.75(1 \mathrm{H}, \mathrm{s}), 4.73(1 \mathrm{H}, \mathrm{s}), 2.34(2 \mathrm{H}, \mathrm{t}, J=8.0 \mathrm{~Hz}), 2.16(2 \mathrm{H}, \mathrm{dt}, J$ $=7.5,7.5 \mathrm{~Hz}), 2.09(2 \mathrm{H}, \mathrm{t}, J=8.0 \mathrm{~Hz}), 2.05(2 \mathrm{H}, \mathrm{t}, J=8.0 \mathrm{~Hz}), 1.76(2 \mathrm{H}, \mathrm{tt}, J=7.5,7.5 \mathrm{~Hz}) ;{ }^{13} \mathrm{C}$ NMR (100 MHz, $\left.\mathrm{CDCl}_{3}\right) \delta 180.4,147.9,138.5,114.8,110.2,35.4,35.3,33.7,32.1,22.7$; HRMS (EI) $\mathrm{m} / e$ calcd $\left(\mathrm{M}^{+}\right)$169.1233, found 169.1229.<smiles>C=C(CCCCO[SbH3])CCCCC(=O)O</smiles>

9-(tert-Butyl-dimethyl-silanyloxy)-5-methylene-nonanoic acid: Flash Chromatography (Hexanes:EtOAc;4:1) yielded a clear liquid (60\%). $\mathrm{R}_{f}=3.20$ (Hexanes:EtOAc;3:1); IR (Thin Film) v 2930, 1710, 1412, 1255, 1104, $836 \mathrm{~cm}^{-1}$; ${ }^{1} \mathrm{H}$ NMR (300 MHz, $\left.\mathrm{CDCl}_{3}\right) \delta 4.77(1 \mathrm{H}, \mathrm{s}), 4.75$ $(1 \mathrm{H}, \mathrm{s}), 3.63(2 \mathrm{H}, \mathrm{t}, J=8.0 \mathrm{~Hz}), 2.37(2 \mathrm{H}, \mathrm{t}, J=7.5 \mathrm{~Hz}), 2.08(2 \mathrm{H}, \mathrm{t}, J=7.5 \mathrm{~Hz}), 2.03(2 \mathrm{H}, \mathrm{t}, J=$ $7.5 \mathrm{~Hz}), 1.80(2 \mathrm{H}, \mathrm{tt}, J=7.5,7.5 \mathrm{~Hz}), 1.60-1.40(4 \mathrm{H}, \mathrm{m}), 0.91(9 \mathrm{H}, \mathrm{s}), 0.06(6 \mathrm{H}, \mathrm{s}) ;{ }^{13} \mathrm{C}$ NMR $\left(100 \mathrm{MHz}, \mathrm{CDCl}_{3}\right) \delta 180.2,148.6,110.0,63.3,35.7,35.3,33.7,32.7,26.2$, 24.2, 22.9, 18.6, -5.0; HRMS (EI) m/e calcd $\left(\mathrm{M}^{+}\right)$301.2199, found 301.2188. 

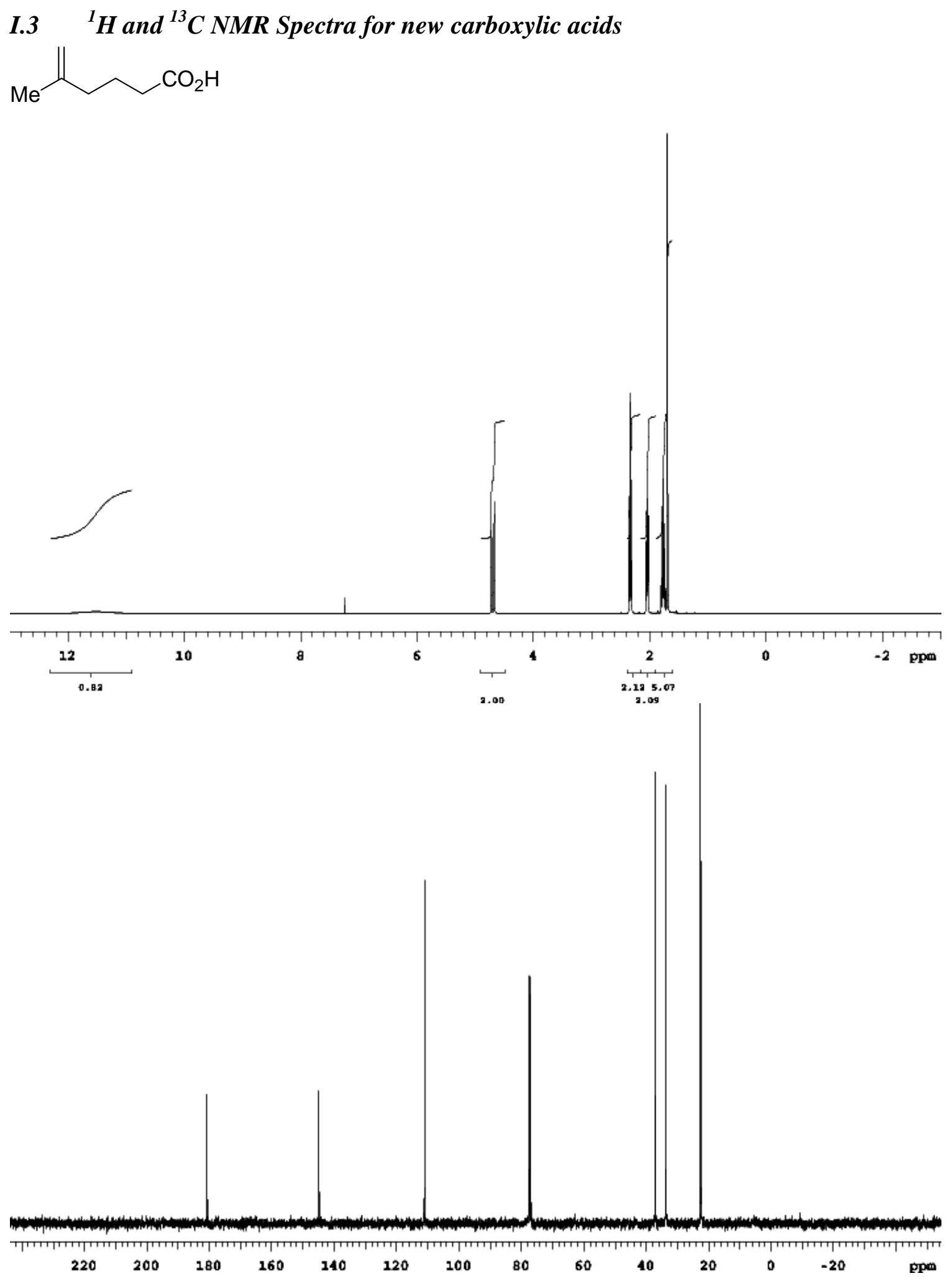
<smiles>C=C(CCCCC(=O)O)CCCC(=O)O</smiles>

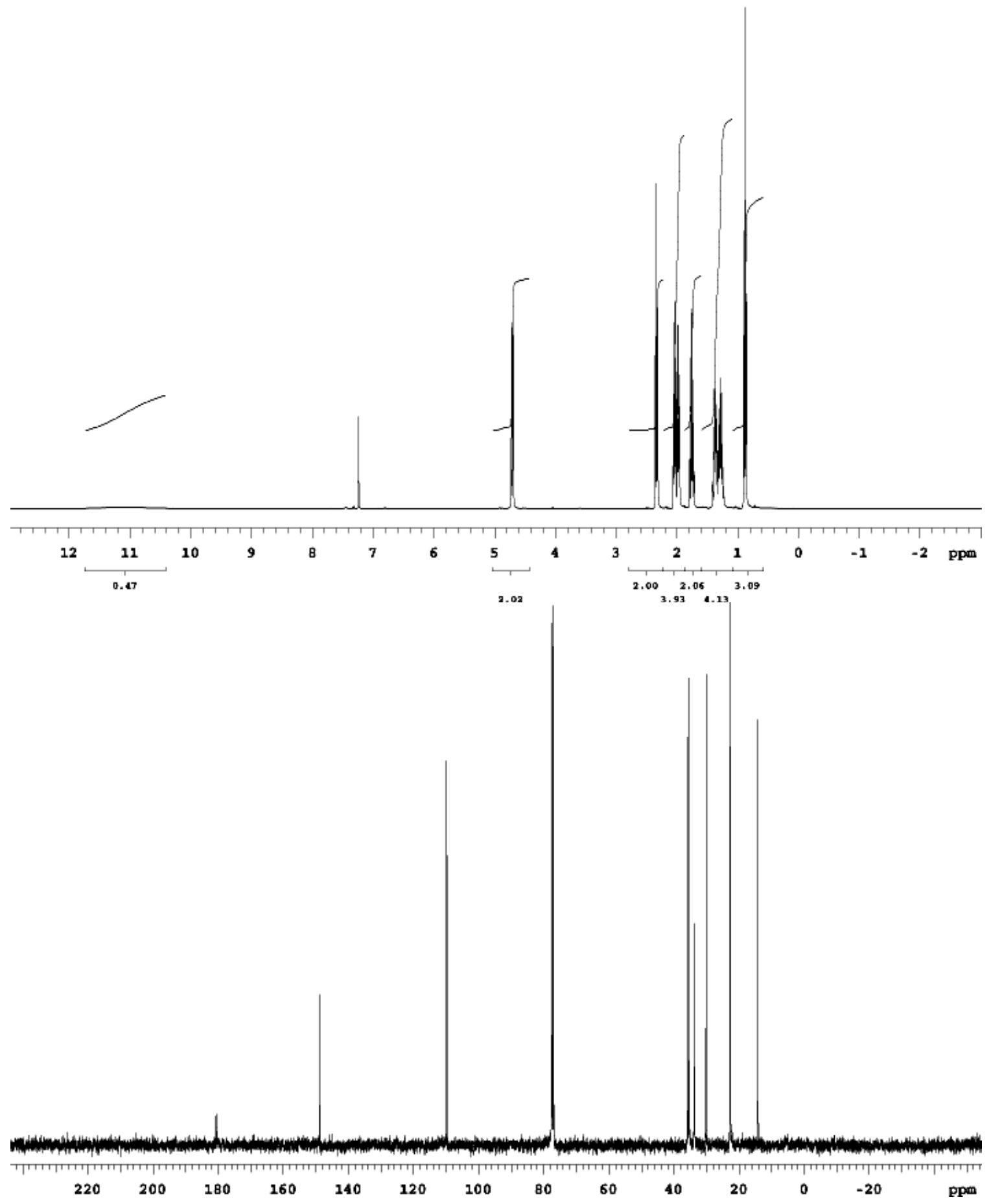



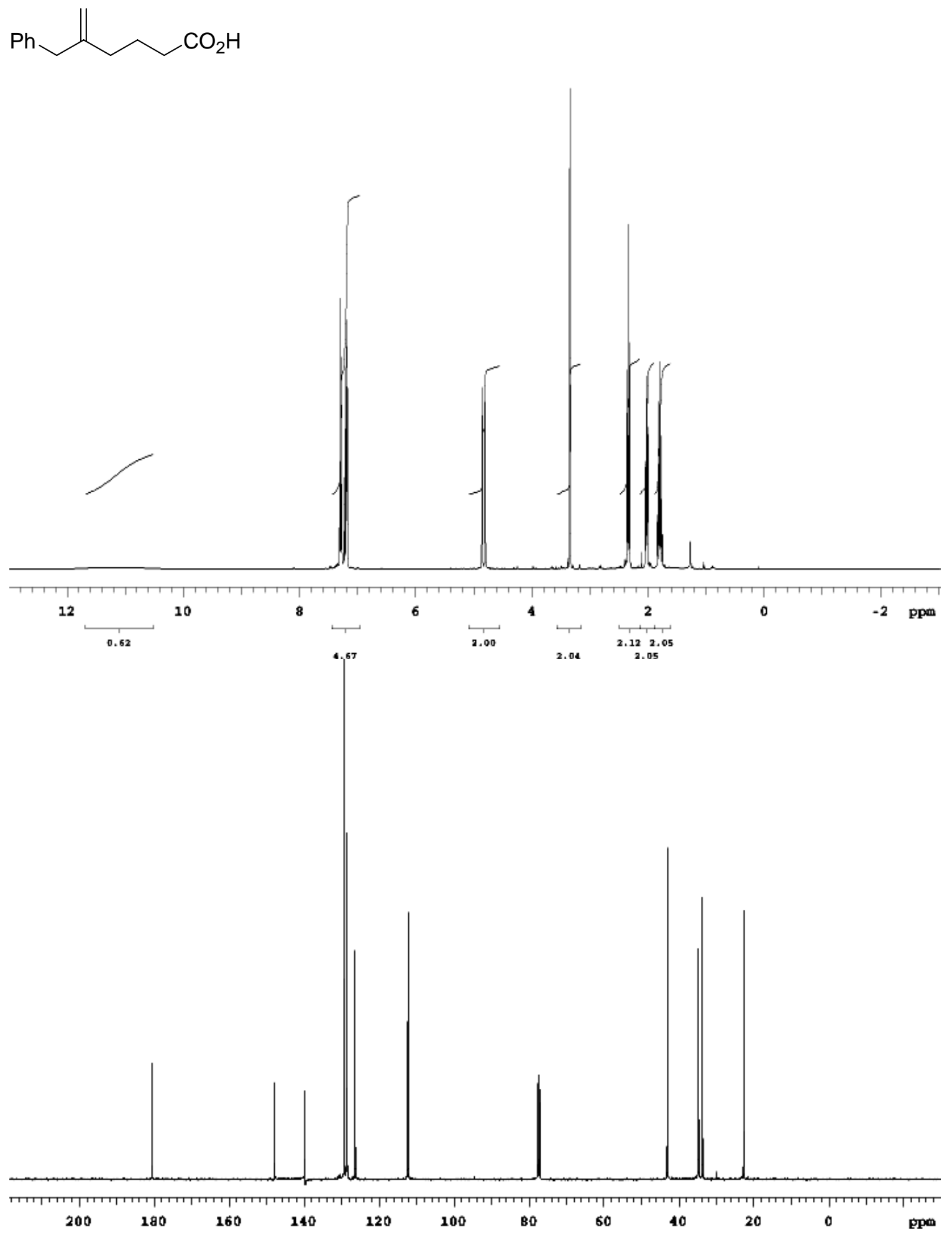
<smiles>C=C(CCCC(=O)O)C(C)C</smiles>
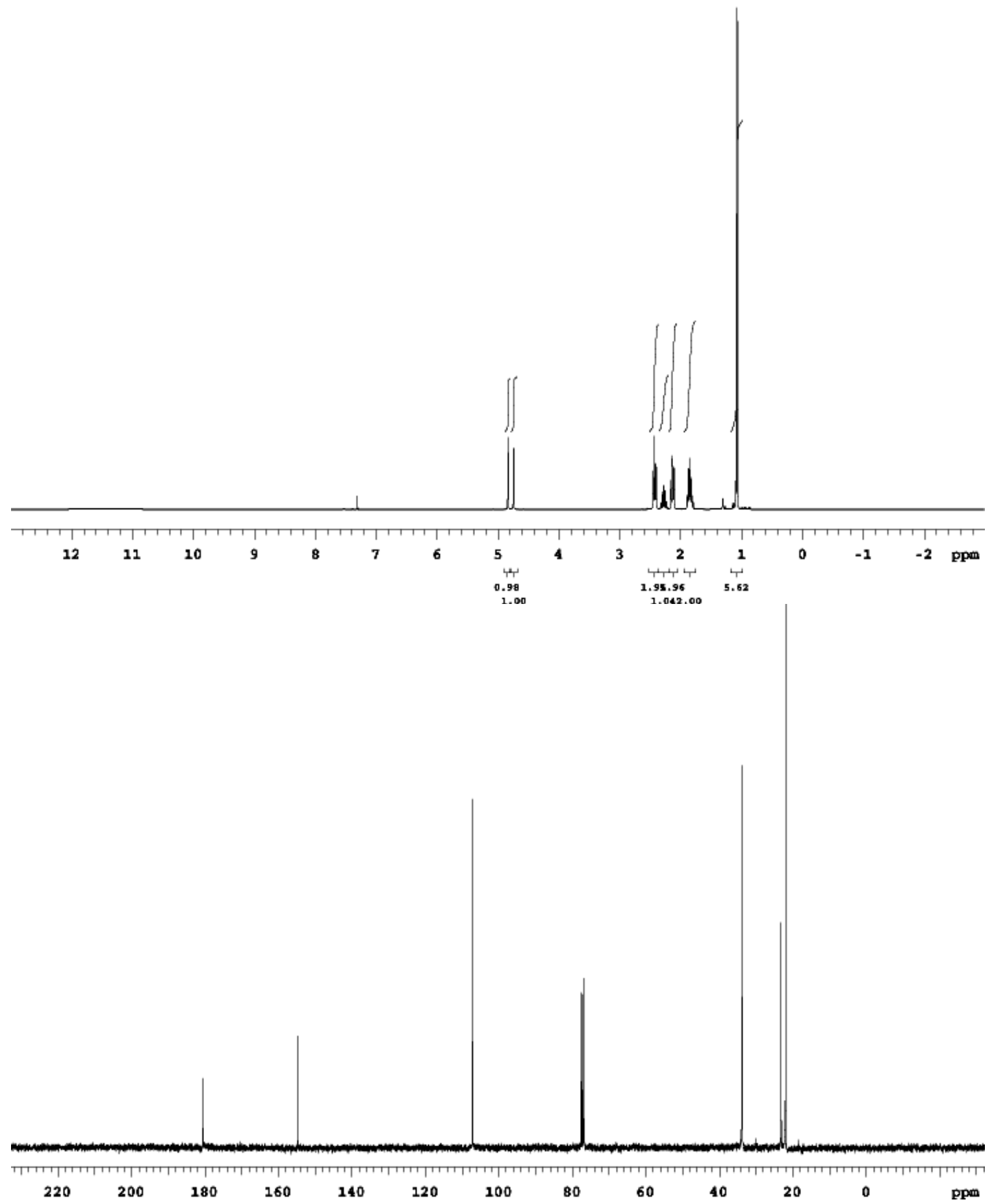
<smiles>C=C(CCCC(=O)O)C1CCCCC1</smiles>
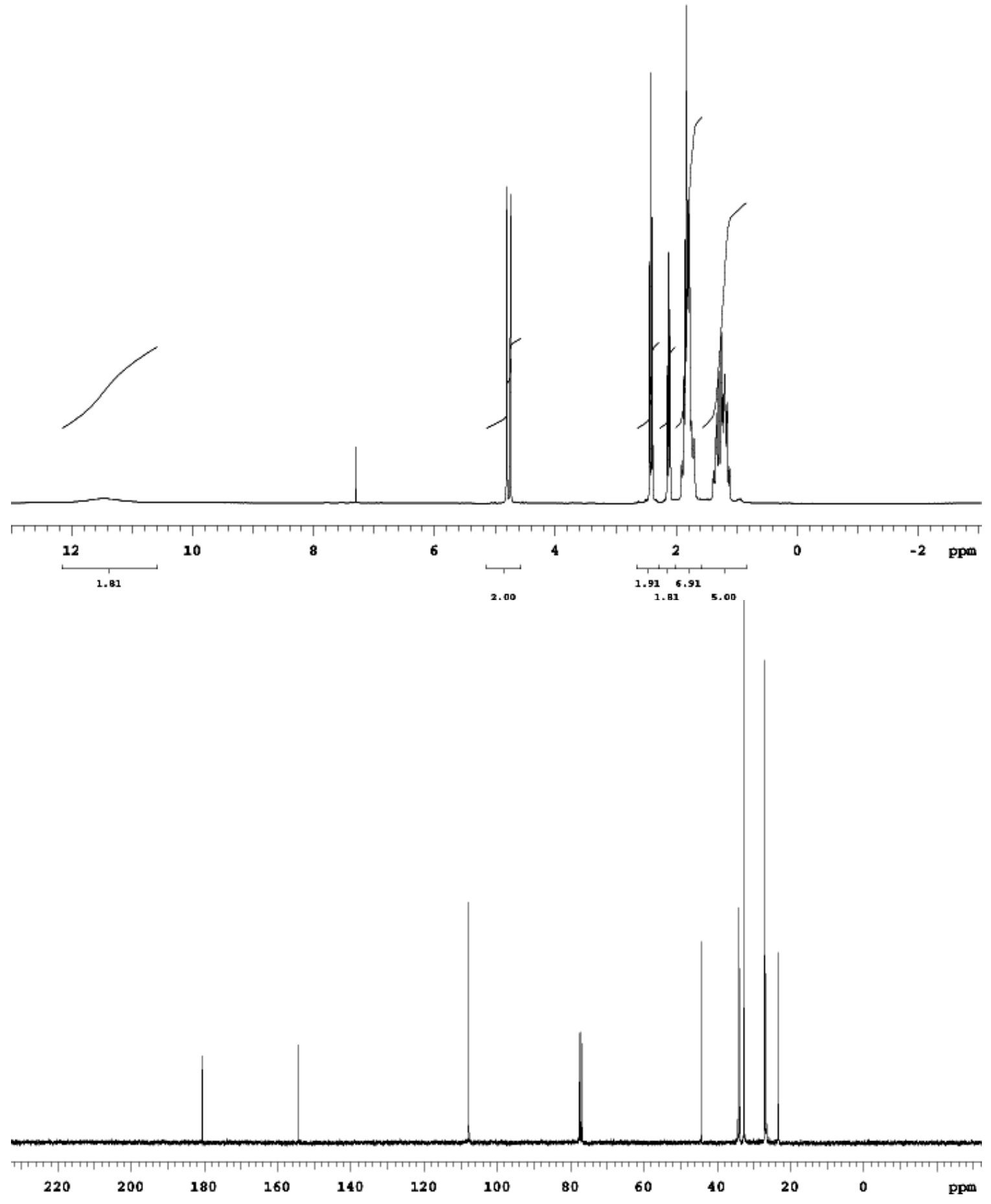


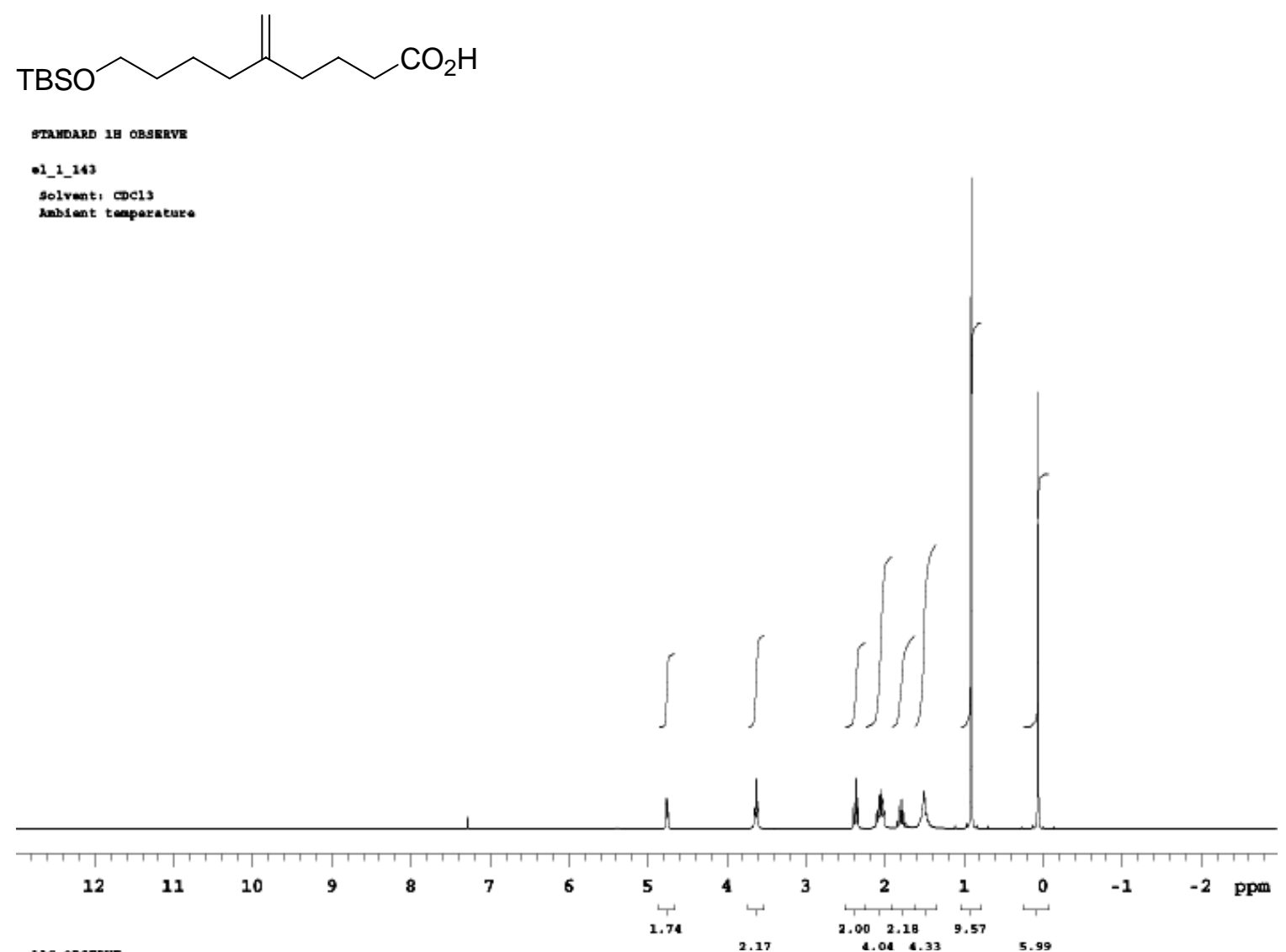

$13 \mathrm{C}$ OBsxavs

Solvent, CDC13

nebient teaperature

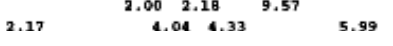

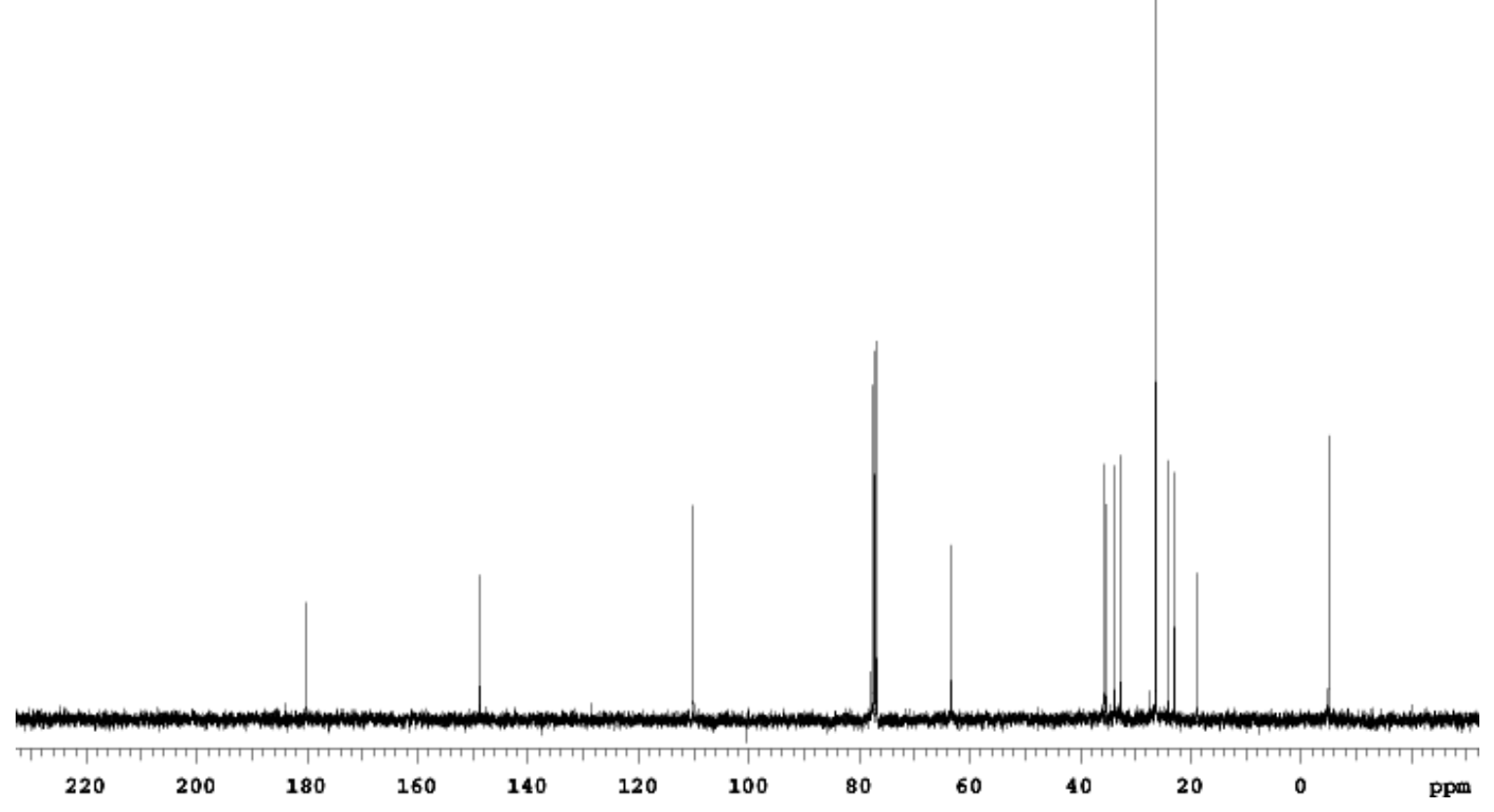




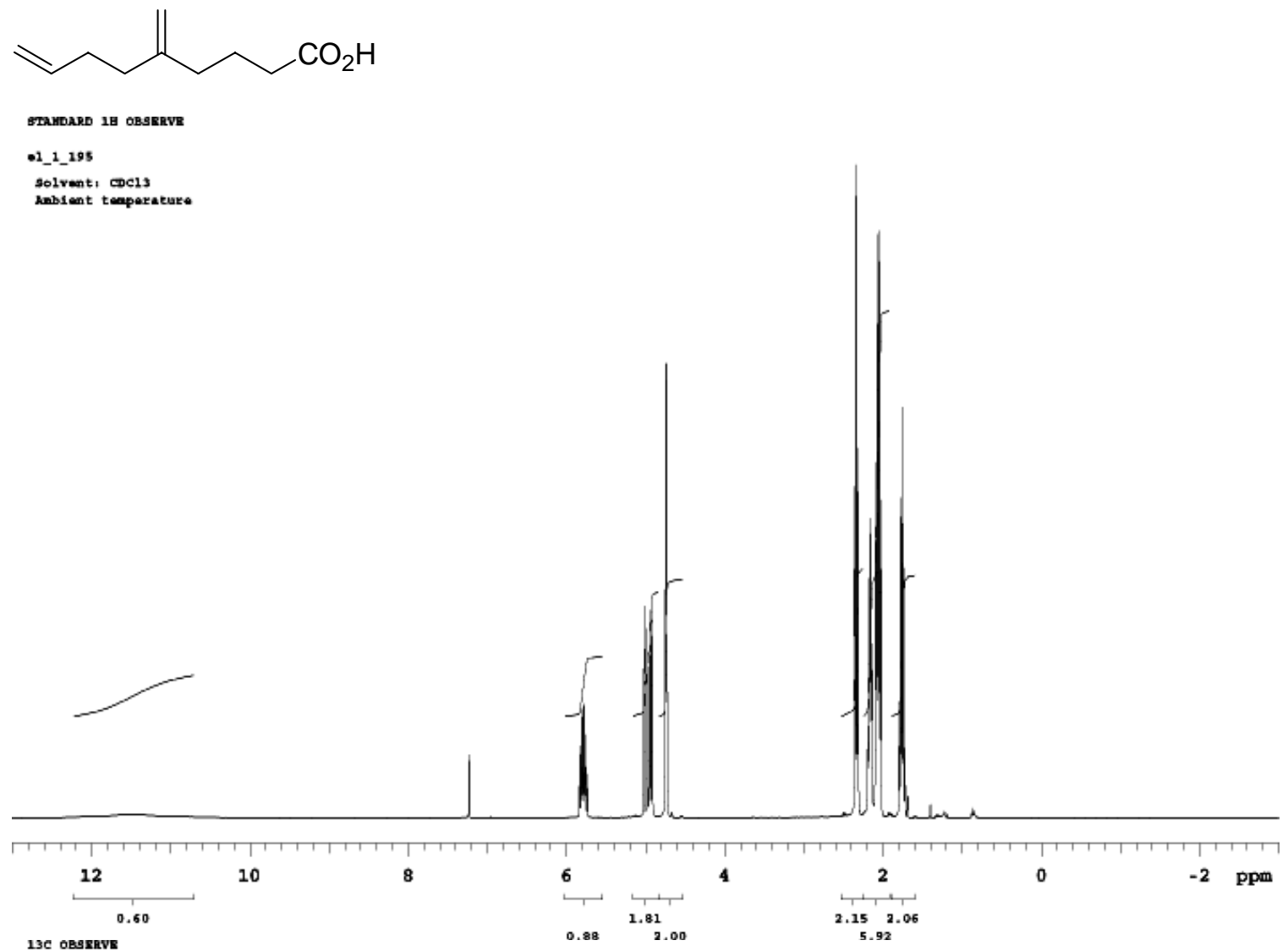

13C OBSPRVE

-1_1_19s

solvent, cDC1s

hablent teaparatur.

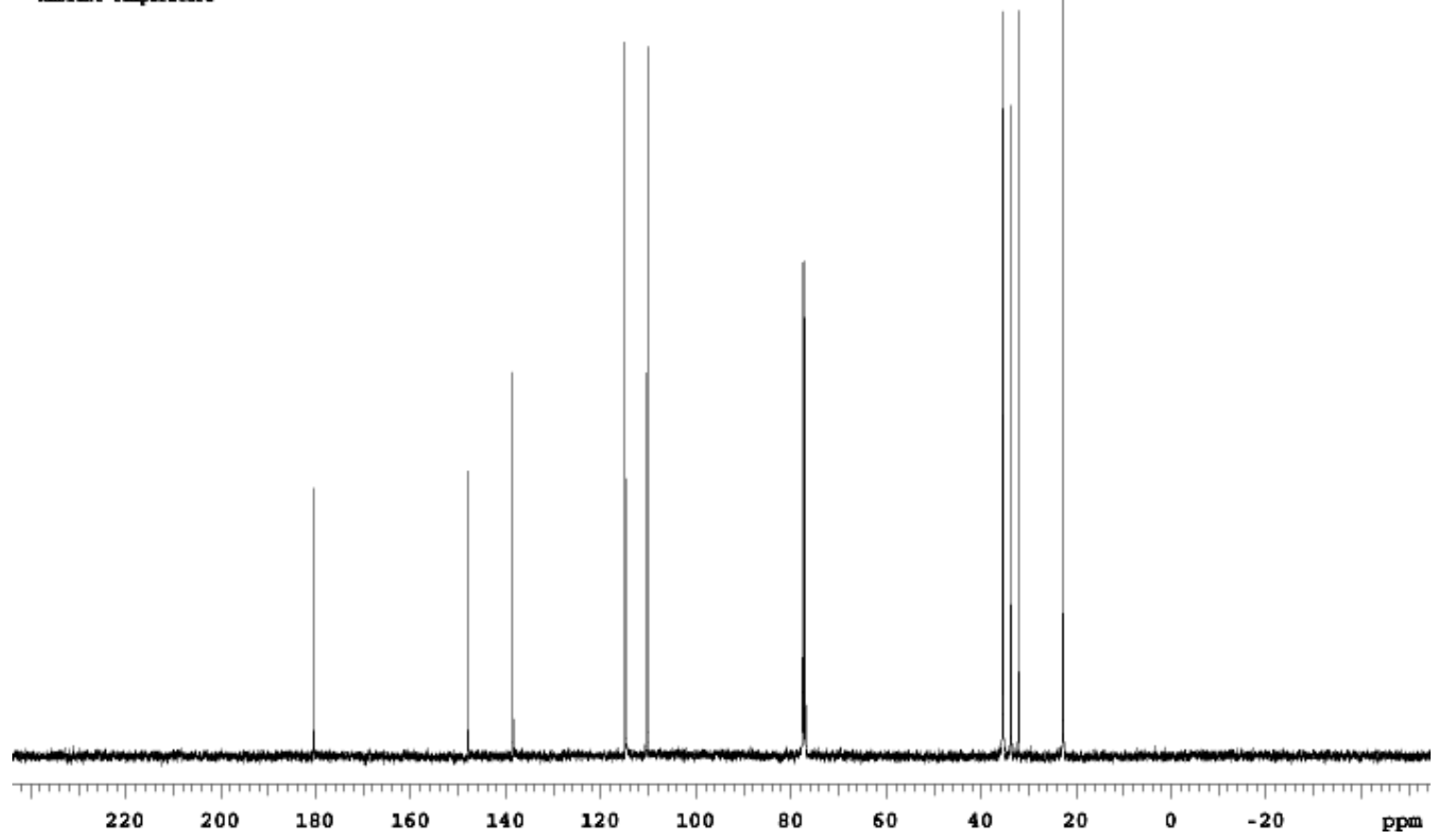




\section{I.4 General procedure for isocyanate synthesis.}

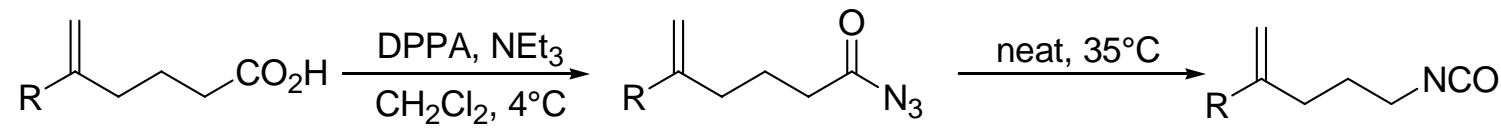

In a flame dried flask under Ar atmosphere, diphenylphosphoryl azide (8.88 mmol, 1.2 eq) was added to a stirring solution of carboxylic acid $(7.4 \mathrm{mmol})$ in dichloromethane $(25 \mathrm{~mL})$ at $4{ }^{\circ} \mathrm{C}$. Triethylamine (8.88 mmol, $1.2 \mathrm{eq})$ was then slowly added. After 4 hours, the reaction was concentrated under vacuum and rapidly purified by flash chromatography (solvent removal was carried out with the rotovap bath temperature less than $23{ }^{\circ} \mathrm{C}$ ). The resulting acyl azide was then gently heated to $35^{\circ} \mathrm{C}$ for $16-24$ hours to afford the desired isocyanate.<smiles>C=C(C)CCCNC=O</smiles>

5-Isocyanato-2-methyl-pent-1-ene (5a): Flash chromatography of the acyl azide (Hex:EtOAc;98:2) and subsequent thermal conversion yielded a clear liquid (78\%). IR (Thin Film) v 2941, 2275, 1651, 1447, 1355, $891 \mathrm{~cm}^{-1}$; ${ }^{1} \mathrm{H}$ NMR (400 MHz, CDCl 3 ) $\delta 4.76(1 \mathrm{H}, \mathrm{s})$, $4.71(1 \mathrm{H}, \mathrm{s}), 3.31(2 \mathrm{H}, \mathrm{t}, J=6.5 \mathrm{~Hz}), 2.11(2 \mathrm{H}, \mathrm{t}, J=7.5 \mathrm{~Hz}), 1.75(2 \mathrm{H}, \mathrm{tt}, J=7.0,7.0 \mathrm{~Hz}), 1.73$ (3H, s); ${ }^{13} \mathrm{C}$ NMR (100 MHz, $\left.\mathrm{CDCl}_{3}\right) \delta 144.2,111.2,42.6,34.7,29.2,22.4$.<smiles>C=C(CC)C(=C)CCC[N+](=O)O</smiles>

1-Isocyanato-4-methylene-octane (5b): Flash chromatography of the acyl azide (Hex:EtOAc;98:2) and subsequent thermal conversion yielded a clear liquid (70\%). IR (Thin Film) v 2929, 2170, 1644, 1489, 1270, 1183, $966 \mathrm{~cm}^{-1} ;{ }^{1} \mathrm{H}$ NMR $\left(400 \mathrm{MHz}, \mathrm{CDCl}_{3}\right) \delta 4.74(1 \mathrm{H}$, s), $4.70(1 \mathrm{H}, \mathrm{s}), 3.28(2 \mathrm{H}, \mathrm{t}, J=6.5 \mathrm{~Hz}), 2.08(2 \mathrm{H}, \mathrm{t}, J=8.0 \mathrm{~Hz}), 1.98(2 \mathrm{H}, \mathrm{t}, J=7.5 \mathrm{~Hz}), 1.72$ $(2 \mathrm{H}, \mathrm{tt}, J=7.5,7.0 \mathrm{~Hz}), 1.43-1.24(4 \mathrm{H}, \mathrm{m}), 0.88(3 \mathrm{H}, \mathrm{t}, J=8.0 \mathrm{~Hz}) ;{ }^{13} \mathrm{C} \mathrm{NMR}\left(100 \mathrm{MHz}, \mathrm{CDCl}_{3}\right)$ $\delta$ 148.3, -653904.0, 42.7, 35.8, 32.9, 30.1, 29.4, 22.6, 14.2.<smiles>C=C(CCC[N+]=O)CC(C)C</smiles>

1-Isocyanato-6-methyl-4-methylene-heptane (5c): Flash chromatography of the acyl azide (Hex:EtOAc;98:2) and subsequent thermal conversion yielded a clear liquid (54\%). IR (Thin Film) v 2955, 2870, 2277, 1644, 1465, $1367 \mathrm{~cm}^{-1} ;{ }^{1} \mathrm{H}$ NMR (400 MHz, CDCl $\left.{ }_{3}\right) \delta 4.73(1 \mathrm{H}, \mathrm{s})$, $4.71(1 \mathrm{H}, \mathrm{s}), 3.27(2 \mathrm{H}, \mathrm{td}, J=6.5,0.5 \mathrm{~Hz}), 2.04(2 \mathrm{H}, \mathrm{t}, J=7.0 \mathrm{~Hz}), 1.85(2 \mathrm{H}, \mathrm{d}, J=7.0 \mathrm{~Hz})$, 1.75-1.66 (3H, m), $\left.0.84(2 \mathrm{H}, \mathrm{d}, J=6.5 \mathrm{~Hz}) ;{ }^{13} \mathrm{C} \mathrm{NMR} \mathrm{(100} \mathrm{MHz,} \mathrm{CDCl}_{3}\right) \delta 147.0,111.4,45.9$, 42.7, 32.6, 29.3, 26.2, 22.6.<smiles>C=C(CCCN=O)Cc1ccccc1</smiles> 
(5-Isocyanato-2-methylene-pentyl)-benzene (5d): Flash chromatography of the acyl azide (Hex:EtOAc;98:2) and subsequent thermal conversion yielded a clear liquid (75\%). IR (Thin Film) v 2948, 2277, 1645, 1494, 1452, $897 \mathrm{~cm}^{-1}$; ${ }^{1} \mathrm{H}$ NMR $\left(400 \mathrm{MHz}, \mathrm{CDCl}_{3}\right) \delta$ 7.30-7.15 (5H, m), $4.84(1 \mathrm{H}, \mathrm{s}), 4.81(1 \mathrm{H}, \mathrm{s}), 3.33(2 \mathrm{H}, \mathrm{s}), 3.26(2 \mathrm{H}, \mathrm{t}, J=6.5 \mathrm{~Hz}), 2.04(2 \mathrm{H}, \mathrm{t}, J=7.5 \mathrm{~Hz}), 1.72$ $(2 \mathrm{H}, \mathrm{tt}, J=7.0 \mathrm{~Hz}) ;{ }^{13} \mathrm{C}$ NMR $\left(100 \mathrm{MHz}, \mathrm{CDCl}_{3}\right) \delta 147.4,139.5,129.1,128.6,126.5,112.4$, 43.2, 42.6, 32.3, 29.2.

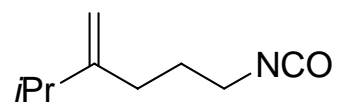

1-Isocyanato-5-methyl-4-methylene-hexane (5e): Flash chromatography of the acyl azide (Hex:EtOAc;98:2) and subsequent thermal conversion yielded a clear liquid (80\%). IR (Thin Film) v 2930, 2852, 2270, 1641, 1448, 1355, $888 \mathrm{~cm}^{-1}$; ${ }^{1} \mathrm{H}$ NMR $\left(400 \mathrm{MHz}, \mathrm{CDCl}_{3}\right) \delta 4.78(1 \mathrm{H}$, s), $4.66(1 \mathrm{H}, \mathrm{s}), 3.30(2 \mathrm{H}, \mathrm{t}, J=6.5 \mathrm{~Hz}), 2.20(1 \mathrm{H}$, sept, $J=7.0 \mathrm{~Hz}), 2.09(2 \mathrm{H}, \mathrm{t}, J=8.0 \mathrm{~Hz}), 1.74$ $(2 \mathrm{H}, \mathrm{tt}, J=7.0,7.0 \mathrm{~Hz}), 1.00(6 \mathrm{H}, \mathrm{d}, J=7.0 \mathrm{~Hz}) ;{ }^{13} \mathrm{C} \mathrm{NMR}\left(100 \mathrm{MHz}, \mathrm{CDCl}_{3}\right) \delta 154.4,107.5$, 42.8, 33.9, 31.3, 29.7, 22.0 .<smiles>C=C(CCCN=O)C1CCCCC1</smiles>

(4-Isocyanato-1-methylene-butyl)-cyclohexane (5f): Flash chromatography of the acyl azide (Hex:EtOAc;98:2) and subsequent thermal conversion yielded a clear liquid (77\%). IR (Thin Film) v 2926, 2852, 2276, 1641, 1448, 1355, $888 \mathrm{~cm}^{-1}$; ${ }^{1} \mathrm{H}$ NMR $\left(400 \mathrm{MHz}, \mathrm{CDCl}_{3}\right) \delta 4.74(1 \mathrm{H}$, s), $4.67(1 \mathrm{H}, \mathrm{s}), 3.28(2 \mathrm{H}, \mathrm{t}, J=7.5 \mathrm{~Hz}), 2.08(2 \mathrm{H}, \mathrm{t}, J=7.5 \mathrm{~Hz}), 1.84-1.62(8 \mathrm{H}, \mathrm{m}), 1.30-1.06$ (5H, m); ${ }^{13} \mathrm{C}$ NMR (100 MHz, $\left.\mathrm{CDCl}_{3}\right) \delta 153.7,108.0,44.2$, 42.8, 32.6, 31.8, 29.8, 27.0, 26.5.<smiles>C=C(CCCNC=O)CO[Bi]</smiles>

(5-Isocyanato-2-methylene-pentyloxymethyl)-benzene (5g): Flash chromatography of the acyl azide (Hex:EtOAc;98:2) and subsequent thermal conversion yielded a clear liquid (61\%). IR (Thin Film) v 2933, 2856, 2277, 1650, 1453, 1096, 1072, $906 \mathrm{~cm}^{-1}$; ${ }^{1} \mathrm{H}$ NMR (400 MHz, $\mathrm{CDCl}_{3}$ )

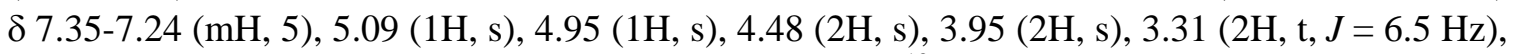
$2.18(2 \mathrm{H}, \mathrm{t}, J=7.0 \mathrm{~Hz}), 1.76(2 \mathrm{H}, \mathrm{tt}, J=7.0,7.0 \mathrm{~Hz}) ;{ }^{13} \mathrm{C} \mathrm{NMR}\left(100 \mathrm{MHz}, \mathrm{CDCl}_{3}\right) \delta 144.6$, 138.4, 128.6, 127.9, 127.9, 113.1, 73.1, 72.2, 42.7, 30.2, 29.2.<smiles></smiles>

tert-Butyl-(8-isocyanato-5-methylene-octyloxy)-dimethyl-silane (5i): Flash chromatography of the acyl azide (Hex:EtOAc;98:2) and subsequent thermal conversion yielded a clear liquid (59\%). IR (Thin Film) v 2931, 2858, 2277, 1472, 1256, 1103, 909, $734 \mathrm{~cm}^{-1}$; ${ }^{1} \mathrm{H}$ NMR (400 $\left.\mathrm{MHz}, \mathrm{CDCl}_{3}\right) \delta 4.75(1 \mathrm{H}, \mathrm{s}), 4.72(1 \mathrm{H}, \mathrm{s}), 3.59(2 \mathrm{H}, \mathrm{t}, J=6.5 \mathrm{~Hz}), 3.28(2 \mathrm{H}, \mathrm{t}, J=6.5 \mathrm{~Hz}), 2.08$ $(2 \mathrm{H}, \mathrm{t}, J=7.5 \mathrm{~Hz}), 2.00(2 \mathrm{H}, \mathrm{t}, J=6.5 \mathrm{~Hz}), 1.72(2 \mathrm{H}, \mathrm{tt}, J=7.5,7.0 \mathrm{~Hz}), 1.51-1.43(4 \mathrm{H}, \mathrm{m}), 0.87$ (9H, s), $0.02(6 \mathrm{H}, \mathrm{s}) ;{ }^{13} \mathrm{C}$ NMR $\left(100 \mathrm{MHz}, \mathrm{CDCl}_{3}\right) \delta 148.1,110.2,63.2,42.7,35.8,32.9,32.6$, 29.3, 26.2, 24.1, 18.6, -5.1.

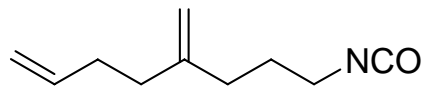


8-Isocyanato-5-methylene-oct-1-ene (5i): Flash chromatography of the acyl azide (Hex:EtOAc;98:2) and subsequent thermal conversion yielded a clear liquid (87\%). IR (Thin Film) v 2936, 2277, 1643, 1446, 1356, $911 \mathrm{~cm}^{-1} ;{ }^{1} \mathrm{H}$ NMR (400 MHz, $\left.\mathrm{CDCl}_{3}\right) \delta 5.78$ (1H, ddd, $J$ $=17.0,10.5,6.5 \mathrm{~Hz}), 4.99(1 \mathrm{H}, \mathrm{dd}, J=17.0,1.5 \mathrm{~Hz}), 4.94(1 \mathrm{H}, \mathrm{dd}, J=10.5,1.5 \mathrm{~Hz}), 4.77(1 \mathrm{H}$, s), $4.74(1 \mathrm{H}, \mathrm{s}), 3.29(2 \mathrm{H}, \mathrm{td}, J=6.5,1.0 \mathrm{~Hz}), 2.17(2 \mathrm{H}$, tdd, $J=7.0,7.0,1.0 \mathrm{~Hz}), 2.11-2.05(4 \mathrm{H}$, m), $1.72(2 \mathrm{H}, \mathrm{tt}, J=7.0 \mathrm{~Hz}) ;{ }^{13} \mathrm{C}$ NMR $\left(100 \mathrm{MHz}, \mathrm{CDCl}_{3}\right) \delta 147.4,138.4,122.2,114.9,110.4$, 42.6, 35.3, 33.0, 32.1, 29.3. 


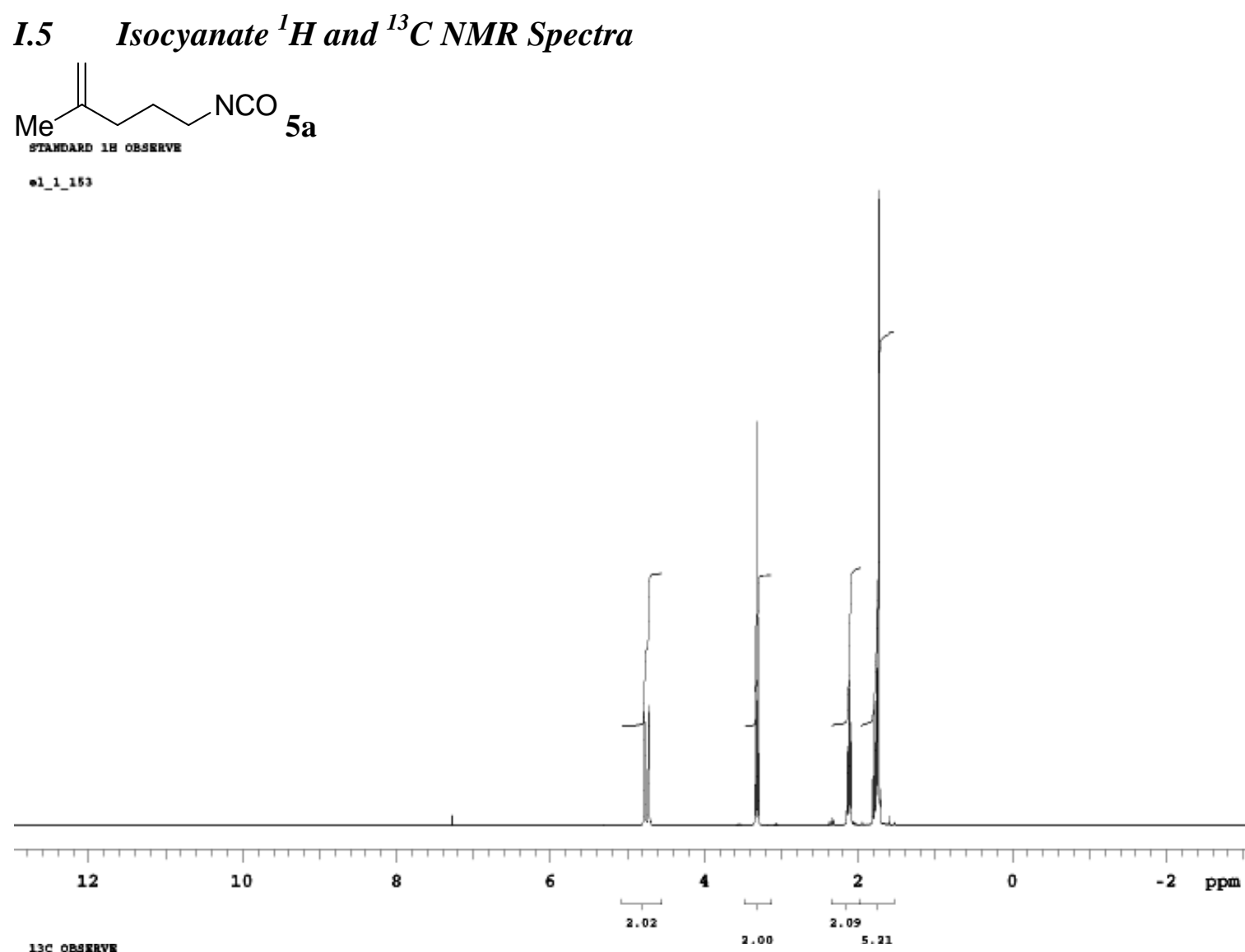

13C OBgYRVE

-1_1_153_13c

solvent, CDC13

hablent teaparakure

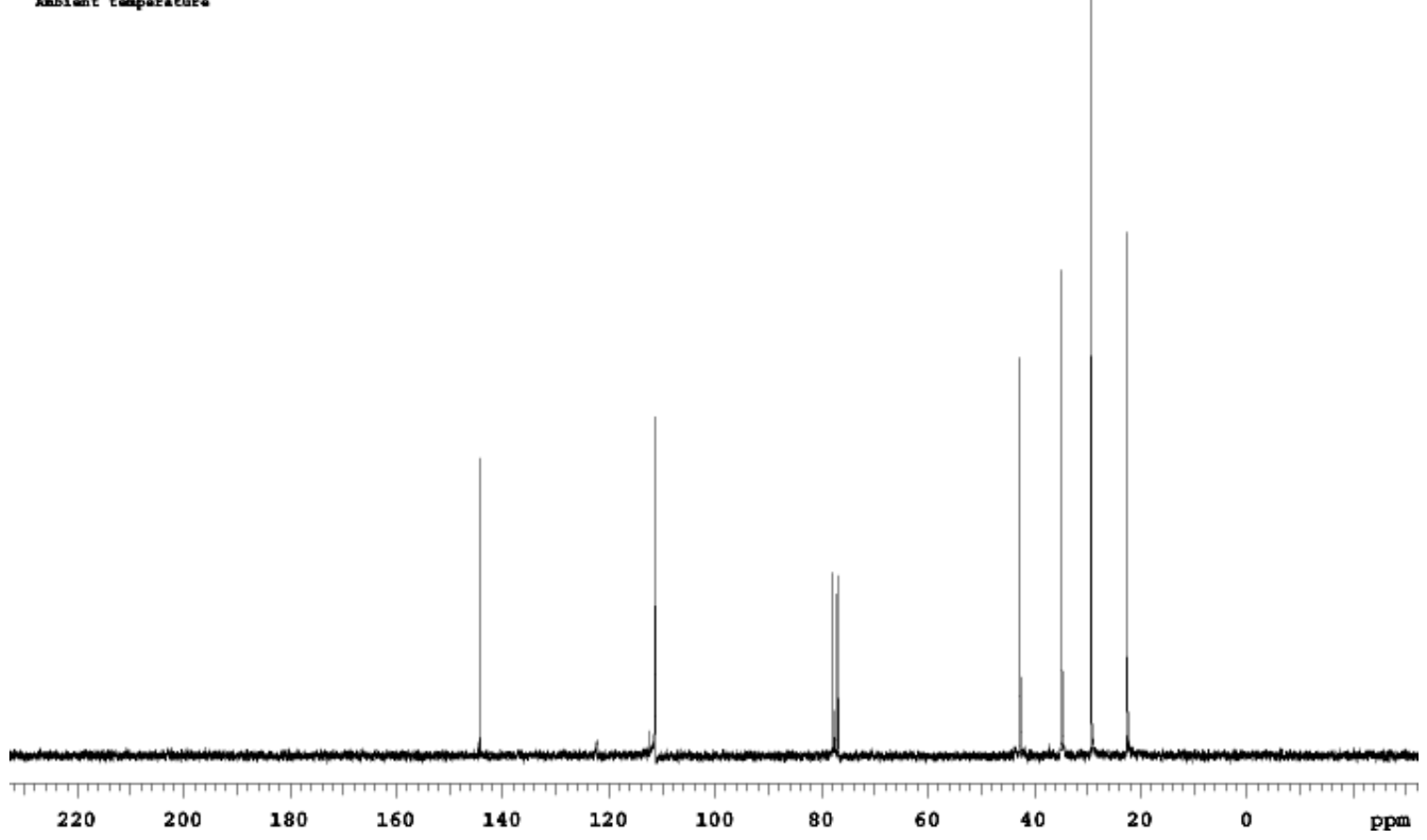


<smiles>C=C(CCCC)CCC[N+]([O-])O</smiles>

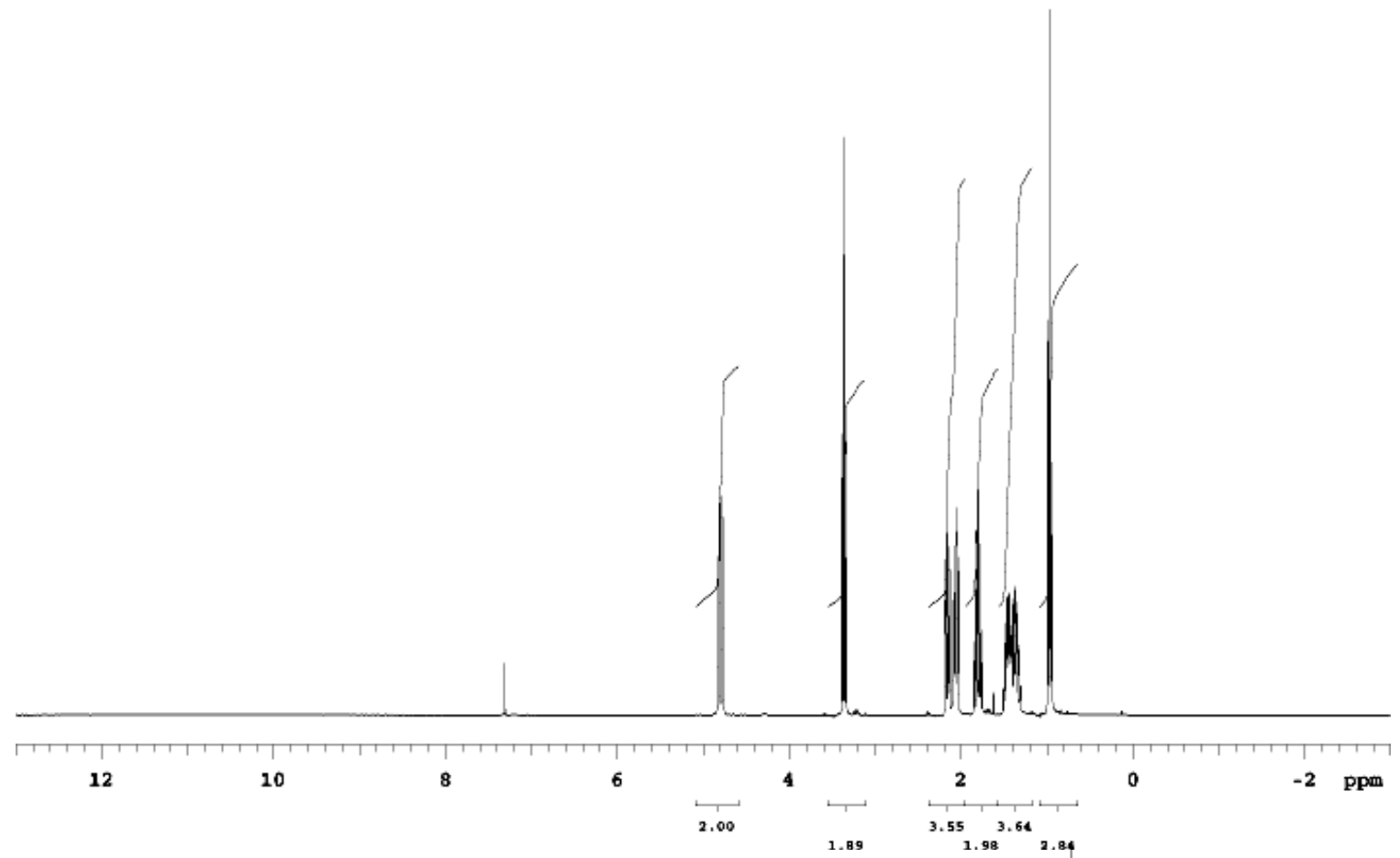


<smiles>C=C(CCC[18O][O-])CCCC(C)C</smiles>
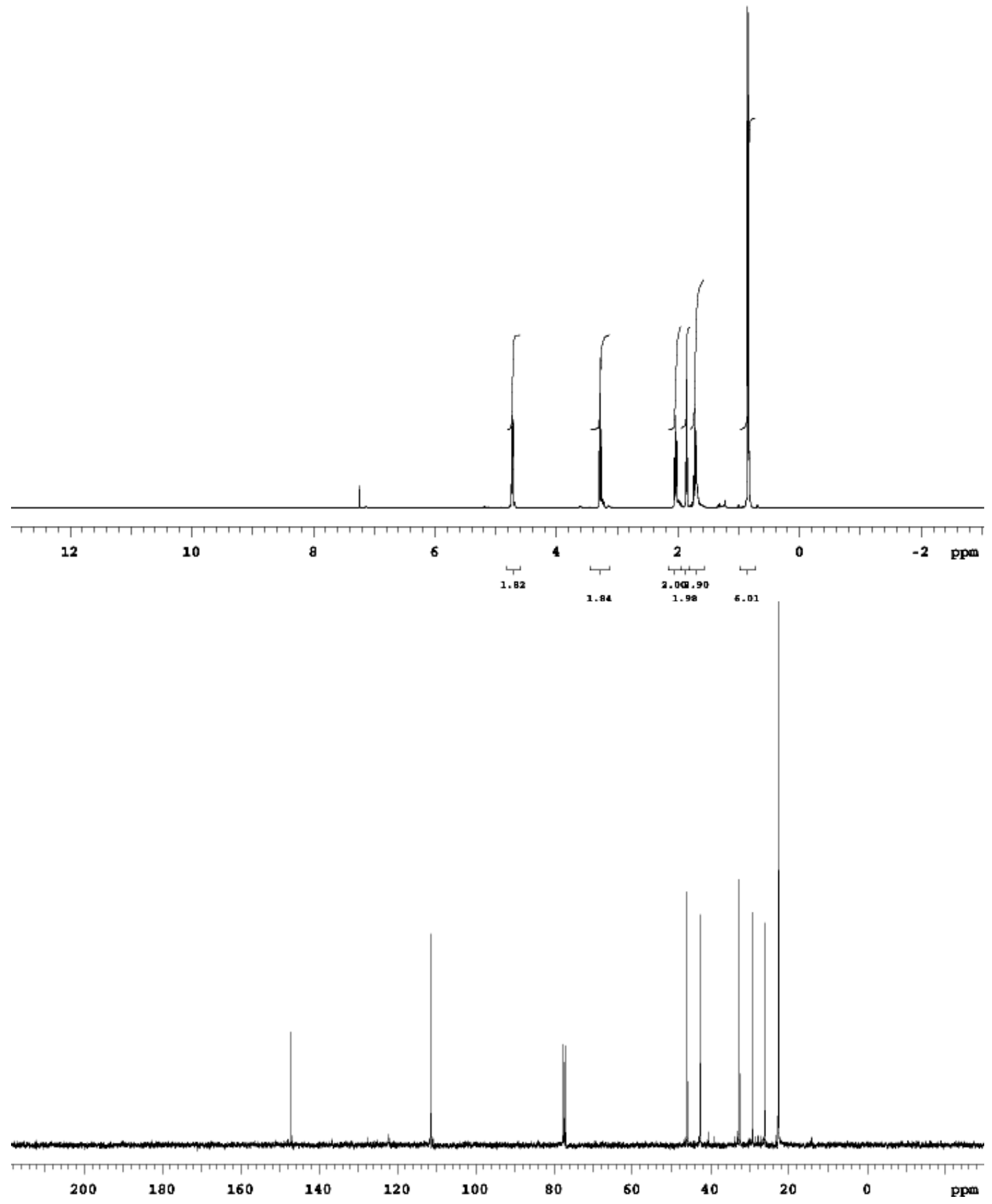

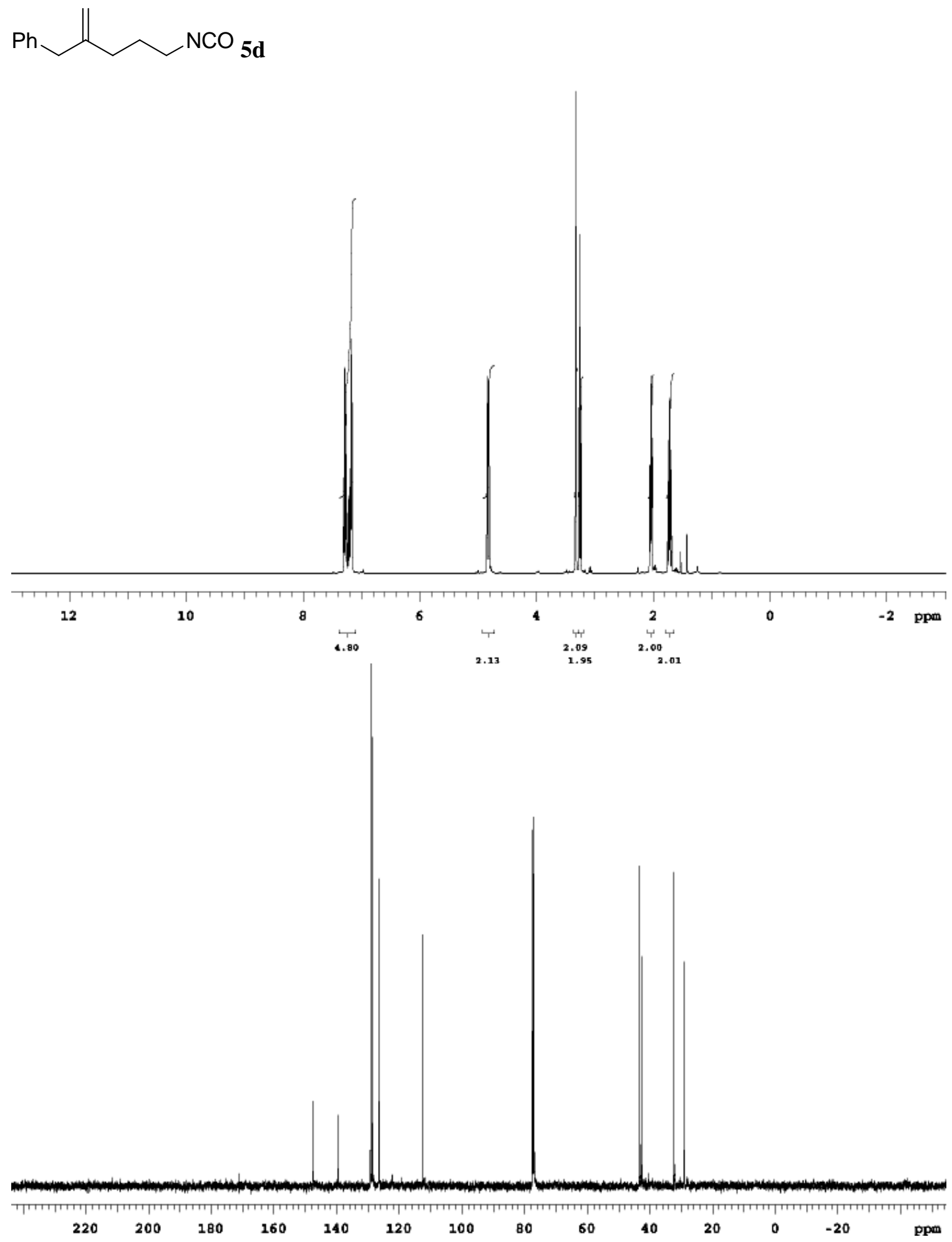
$\mathrm{P}_{\mathrm{Pr}} \mathrm{NCO}_{5 \mathbf{e}}$
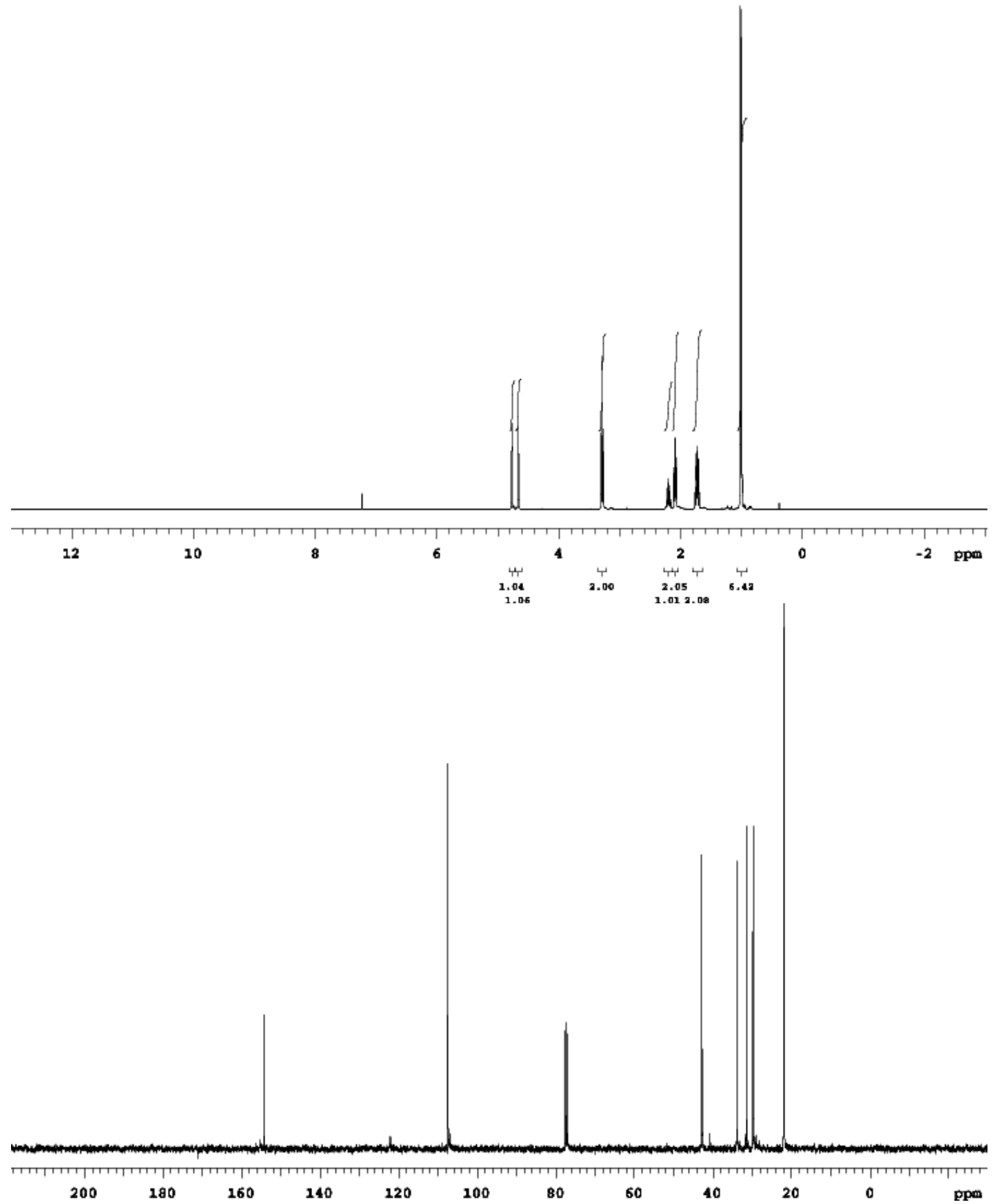
<smiles>C=C(C=O)CCCNC(=O)O[Si]</smiles>
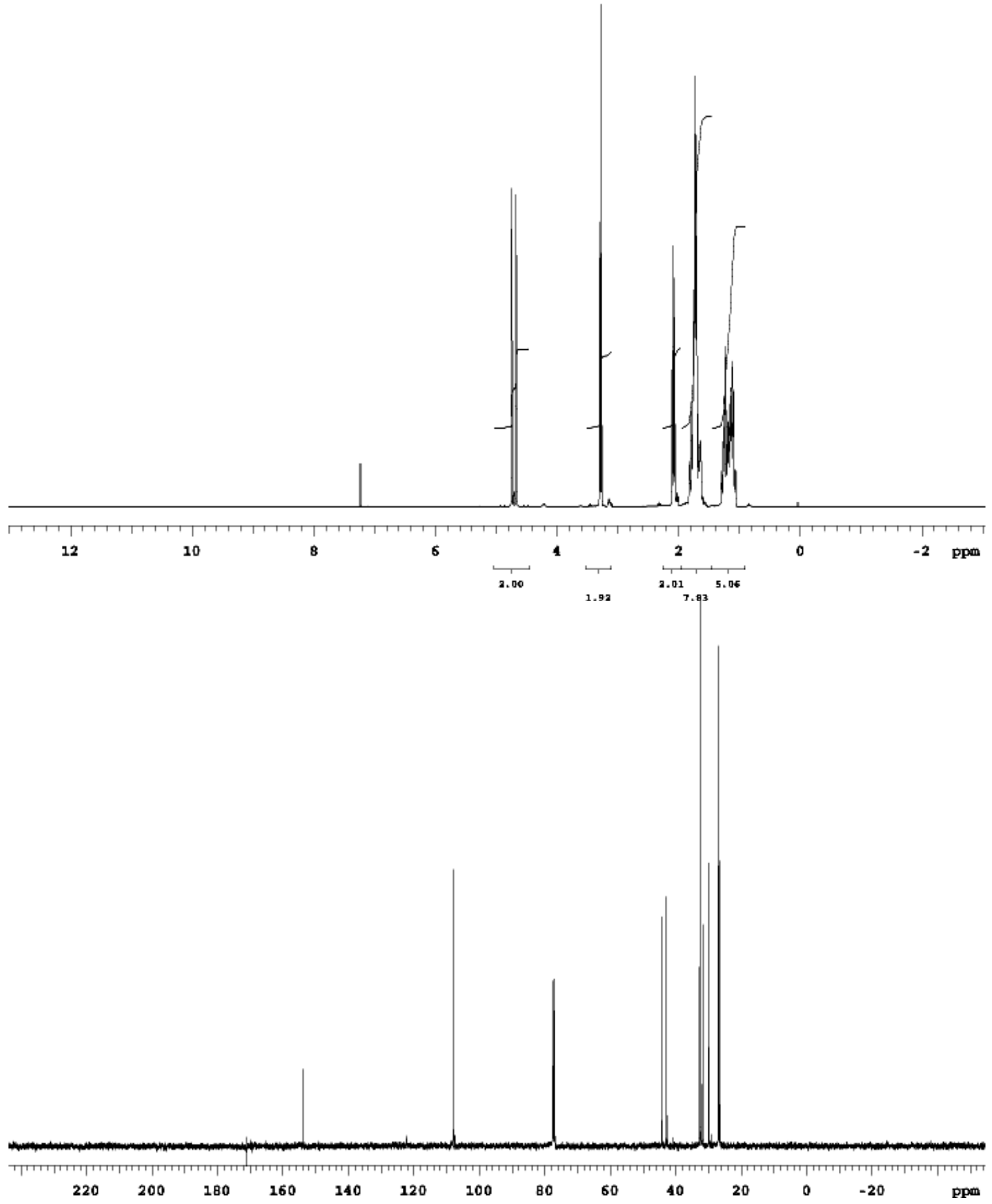
$\mathrm{BnO}^{\sim} \mathrm{NCO}_{5 \mathrm{~g}}$
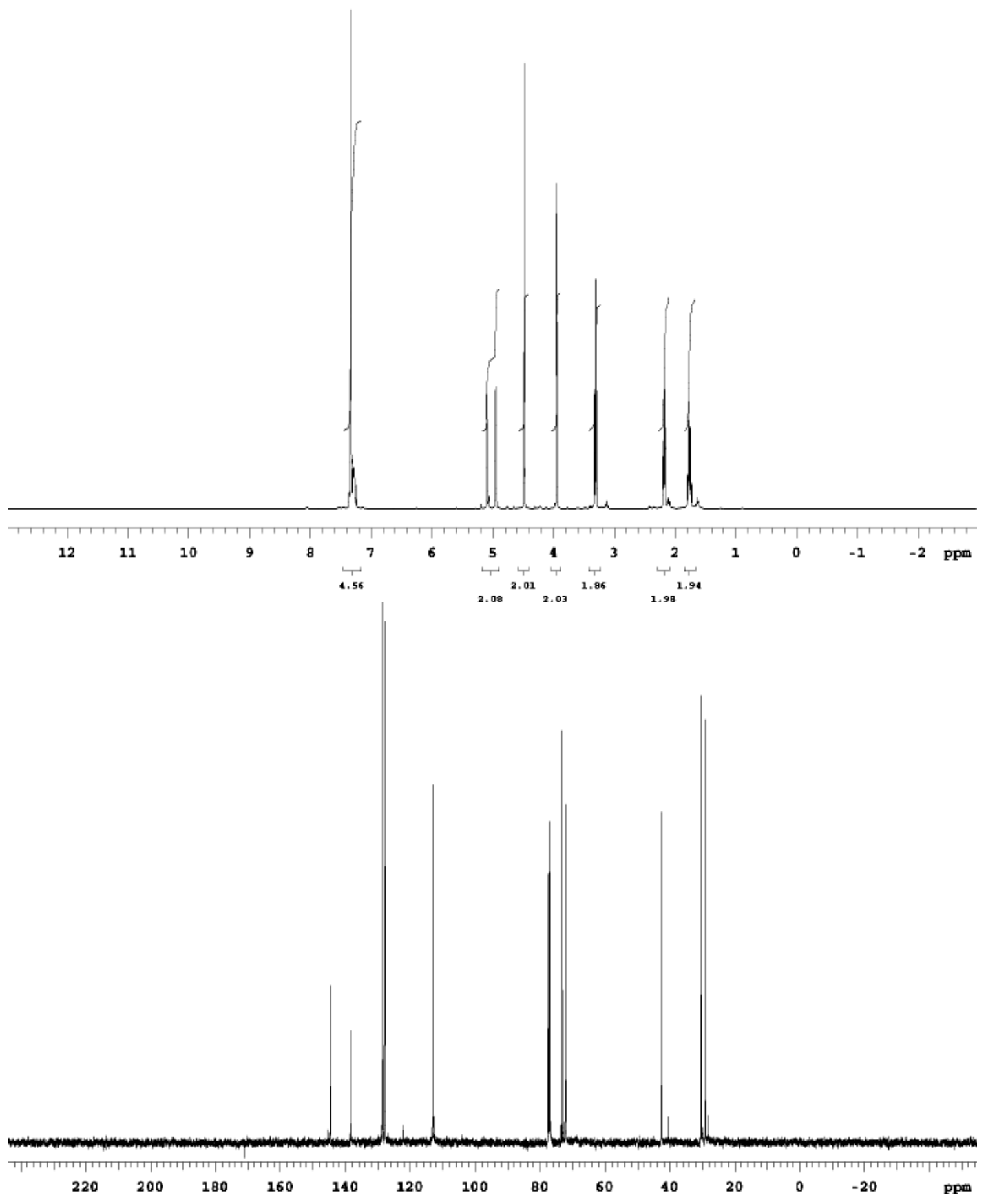


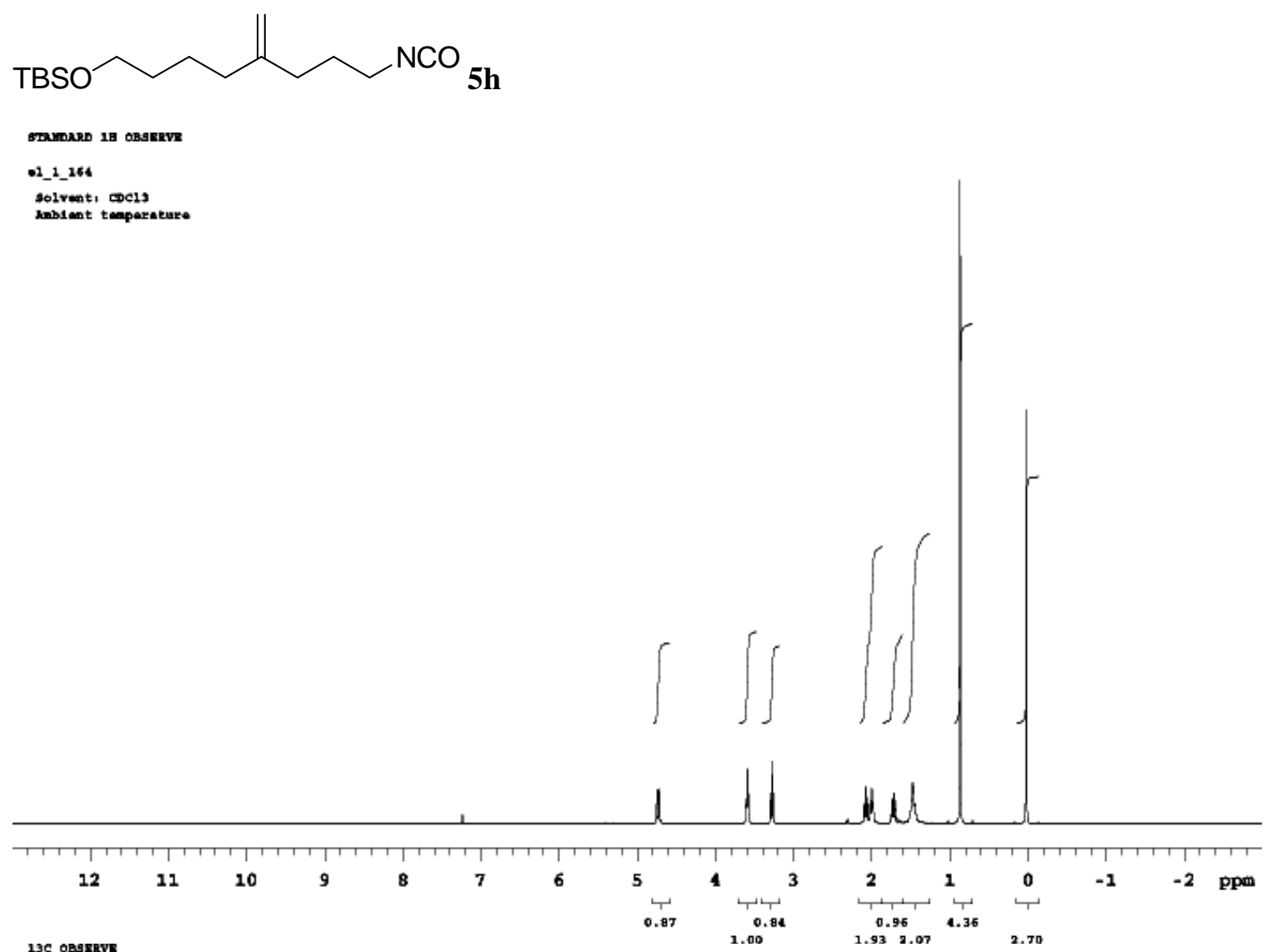

-1_1_264_1

golvent, CDC13

kablent tanparature

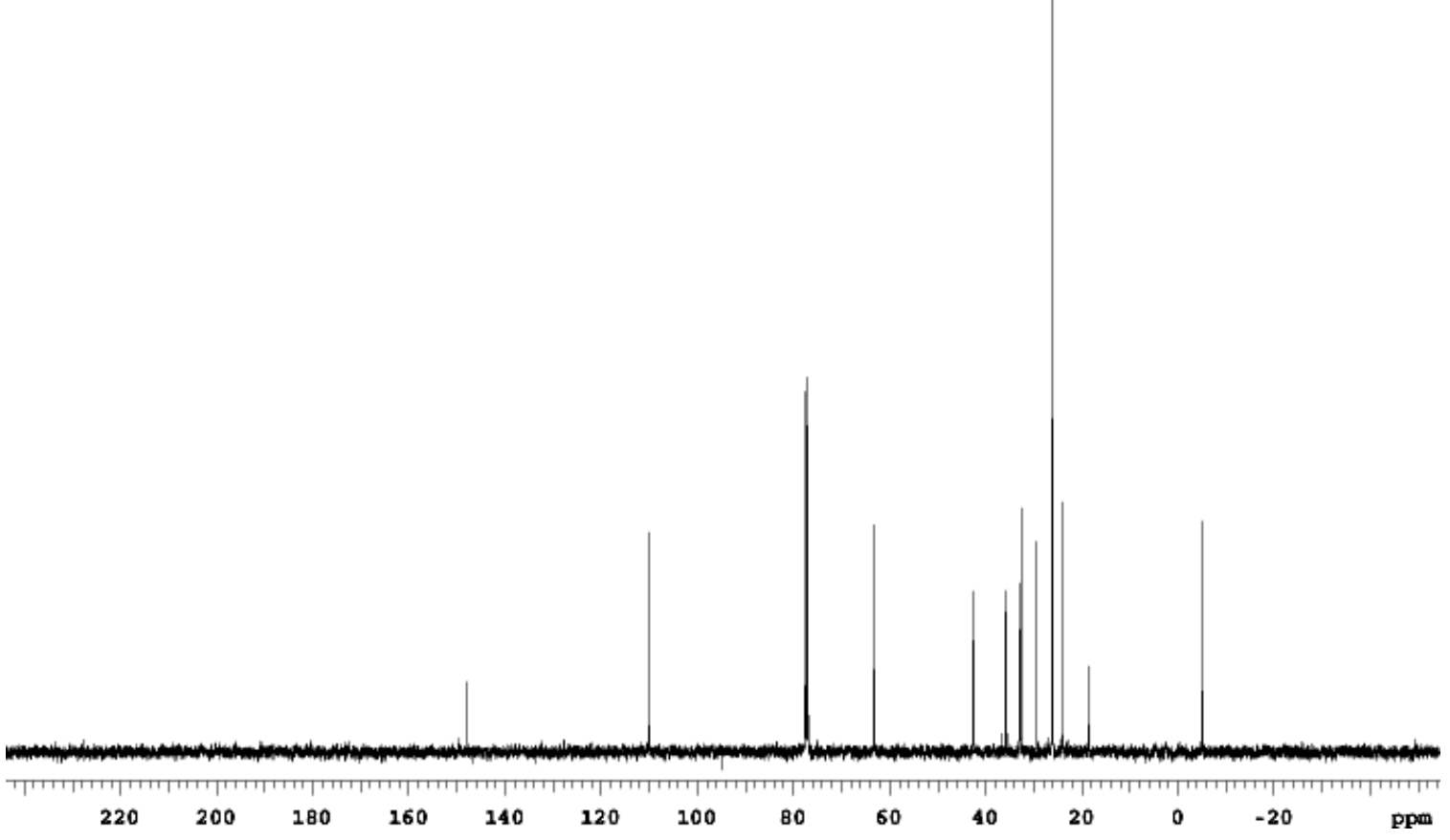




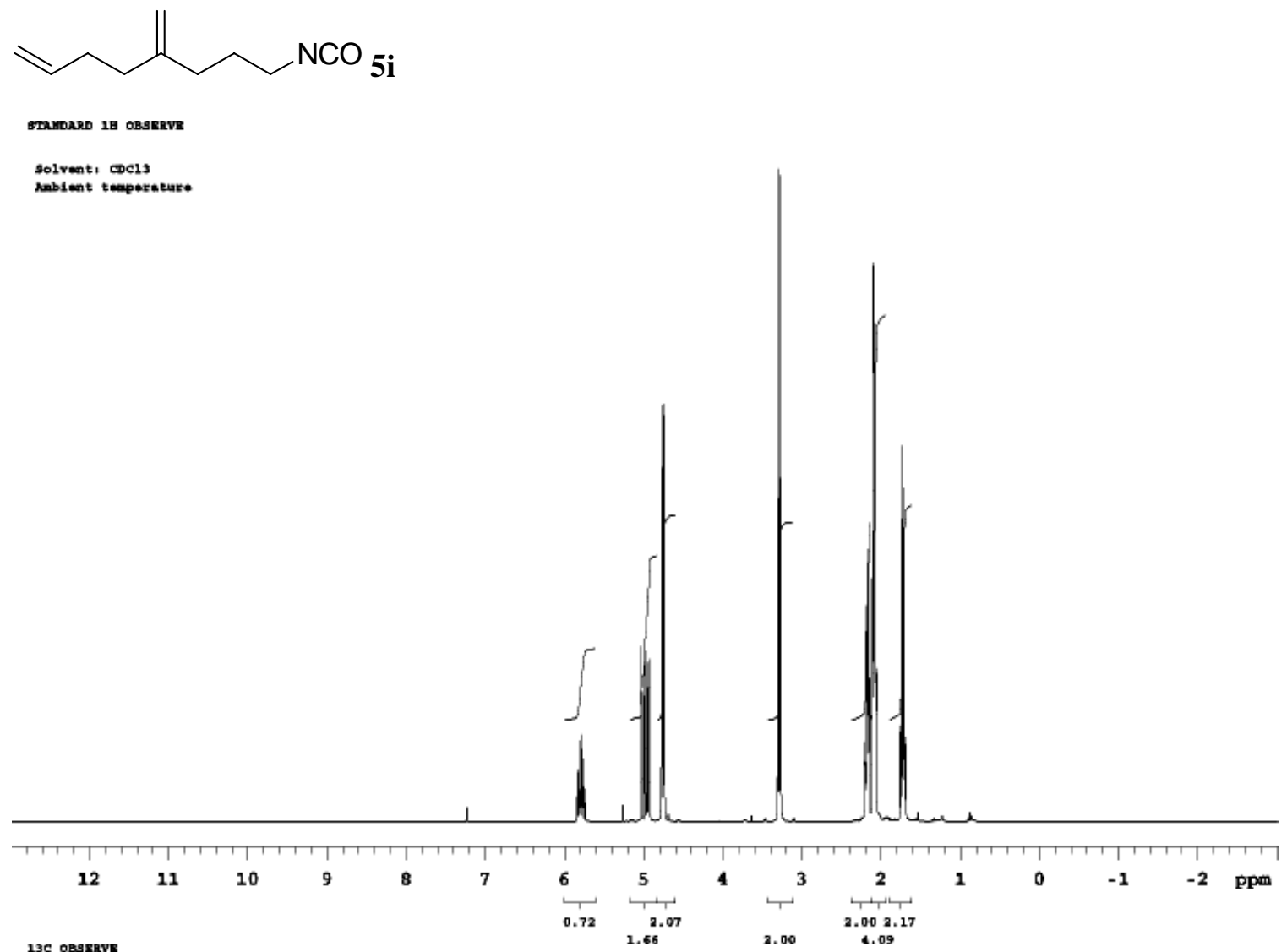

$13 C$ obsyrve

-1_1_194

solvent, CDC1s

hablent teaparakure

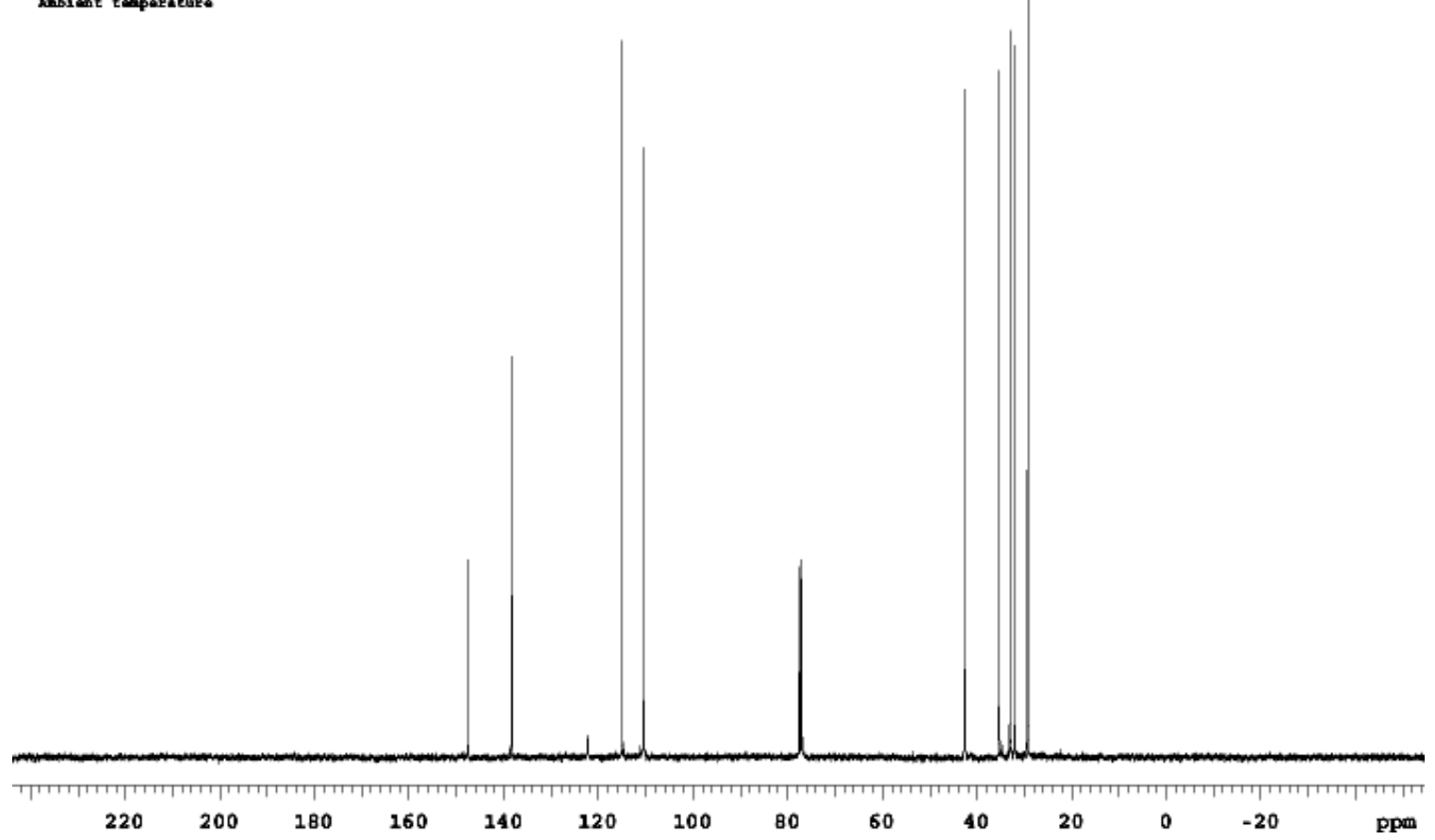




\section{I.6 Synthesis of Ligands}

Procedure A (L3, L4 and L5): to a solution of diol in ether at $0^{\circ} \mathrm{C}$, triethylamine (3 eq) then phosphorous trichloride (1.2 eq) were added. After 6 hours, amine (10 eq) were added, and the reaction warmed to room temperature and left for 2 hours. Filtration, concentration and purification by flash chromatography provides the desired product (after chromatography and concentration, products are dissolved in ether and concentrated again).

Procedure B (alternatively used for L3): to a solution of diol in toluene, hexamethyl phosphorous triamide (1.2 eq) was added. The reaction was then heated at reflux for 48 hours under a slow, dynamic flow of argon. The clear yellow solution was then cooled and diluted with hexanes. The white solid was filtered off, washed with hexanes and dried under vacuum.

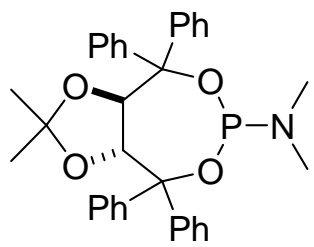

(2,2-Dimethyl-4,4,8,8-tetraphenyl-tetrahydro-[1,3]dioxolo[4,5-e][1,3,2]dioxaphosphepin-6yl)-dimethyl-amine (L3): Flash Chromatography (Hexanes:EtOAc;95:5) yielded a white solid (Procedue A: 54\%, Procedure B without chromatography: 74\%). $\quad \mathrm{R}_{f}=0.50$ (Hexanes:EtOAc;90:10); ${ }^{1} \mathrm{H}$ NMR (400 MHz, $\left.\mathrm{CDCl}_{3}\right) \delta 7.79(2 \mathrm{H}, \mathrm{d}, J=11.0 \mathrm{~Hz}), 7.63(2 \mathrm{H}, \mathrm{d}, J$ $=12.0 \mathrm{~Hz}), 7.51(2 \mathrm{H}, \mathrm{d}, J=11.0 \mathrm{~Hz}), 7.48(2 \mathrm{H}, \mathrm{d}, J=11.0 \mathrm{~Hz}), 7.38-7.20(12 \mathrm{H}, \mathrm{m}), 5.22(1 \mathrm{H}$, dd, $J=12.0,4.5 \mathrm{~Hz}), 4.86(1 \mathrm{H}, \mathrm{d}, J=11.0 \mathrm{~Hz}), 2.76(6 \mathrm{H}, \mathrm{d}, J=14.5 \mathrm{~Hz}), 1.32(3 \mathrm{H}, \mathrm{s}), 0.33(3 \mathrm{H}$, s); ${ }^{13} \mathrm{C}$ NMR $\left(75 \mathrm{MHz}, \mathrm{CDCl}_{3}\right) \delta 147.1,146.7,142.4,142.4,142.0,129.2,129.0,128.9,128.4$, 128.0, 127.8, 127.7, 127.5, 127.4, 127.3, 112.0, 82.8 (d, $J=3.5 \mathrm{~Hz}), 82.5,82.1,81.4$ (d, $J=7.3$ $\mathrm{Hz}), 25.6$ (d, $J=21.0 \mathrm{~Hz}$ ), 27.9, 25.6.

First Report: Keller, E.; Maurer, J.; Naasz, R.; Schader, T.; Meetsma, A.; Feringa, B. L. Tetrahedron Asymmetry, 1998, 9, 2409, 2413.

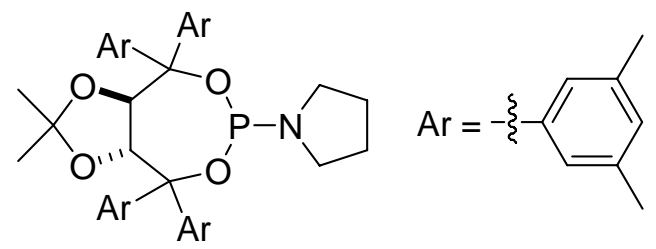

\section{1-[4,4,8,8-Tetrakis-(3,5-dimethyl-phenyl)-2,2-dimethyl-tetrahydro-[1,3]dioxolo[4,5- \\ e][1,3,2]dioxaphosphepin-6-yl]-pyrrolidine (L4): Flash Chromatography} (Hexanes:EtOAc;95:5) yielded a white solid (50\%). $\mathrm{R}_{f}=0.50$ (Hexanes:EtOAc;90:10); IR (Thin Film) v 2917, 2866, 1601, 1456, 1379, 1214, 1159, 1042, $854 \mathrm{~cm}^{-1}$; ${ }^{1} \mathrm{H}$ NMR (400 MHz, $\left.\mathrm{CDCl}_{3}\right)$ $\delta 7.37(2 \mathrm{H}, \mathrm{s}), 7.16(2 \mathrm{H}, \mathrm{s}), 7.04(2 \mathrm{H}, \mathrm{s}), 7.02(2 \mathrm{H}, \mathrm{s}), 6.84(3 \mathrm{H}, \mathrm{s}), 6.80(1 \mathrm{H}, \mathrm{s}), 5.08(1 \mathrm{H}, \mathrm{dd}, J=$ 8.5, $2.5 \mathrm{~Hz}), 4.74(1 \mathrm{H}, \mathrm{d}, J=8.0 \mathrm{~Hz}), 3.47-3.35(2 \mathrm{H}, \mathrm{m}), 3.35-3.15(2 \mathrm{H}, \mathrm{m}), 2.27(6 \mathrm{H}, \mathrm{s}), 2.25$ (12H, s), 2.24 (6H, s), 1.86-1.72 (4H, m), 1.32 (3H, s), 0.25 (3H, s); ${ }^{13} \mathrm{C}$ NMR (100 MHz, $\left.\mathrm{CDCl}_{3}\right)$ 
$\delta 147.2,146.9,142.2,142.1,137.3,136.9,136.7,136.3,129.2,128.9,128.7,127.9,127.0,126.8$, 125.3, 125.2, 111.7, 83.1 (d, $J=4.5 \mathrm{~Hz}$ ), 82.9, 82.7, 81.8, 81.1 (d, $J=5.5 \mathrm{~Hz}$ ), 45.1 (d, $J=19.0$ $\mathrm{Hz}$ ), 27.9, 26.3, 16.2, 25.7, 21.9, 21.8; ${ }^{31} \mathrm{P}$ NMR (75 MHz, $\mathrm{CDCl}_{3}$ ) $\delta 138.40$; HRMS (ESI) m/e calcd $\left(\mathrm{M}+\mathrm{H}^{+}\right)$678.3707, found 678.3702.

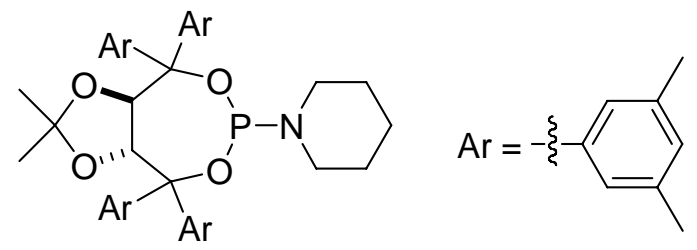

$(R, R)-1-[4,4,8,8$-Tetrakis-(3,5-dimethyl-phenyl)-2,2-dimethyl-tetrahydro-[1,3]dioxolo[4,5-

e][1,3,2]dioxaphosphepin-6-yl]-piperidine (L5): Flash Chromatography (Hexanes:EtOAc;95:5) yielded a white solid (52\%); $[\alpha]_{\mathrm{D}}=-108.0^{\circ}\left(\mathrm{CHCl}_{3}, \mathrm{c}=1.0\right) ; \mathrm{R}_{f}=0.50$ (Hexanes:EtOAc;90:10); IR (Thin Film) v 2931, 2851, 1600, 1448, 1370, 1215, 1159, 1040, $940 \mathrm{~cm}^{-1} ;{ }^{1} \mathrm{H}$ NMR (400 MHz, $\left.\mathrm{CDCl}_{3}\right) \delta 7.39(2 \mathrm{H}, \mathrm{s}), 7.20(2 \mathrm{H}, \mathrm{s}), 7.04(4 \mathrm{H}, \mathrm{s}), 6.84(3 \mathrm{H}, \mathrm{s}), 6.79(1 \mathrm{H}, \mathrm{s}), 5.02(1 \mathrm{H}, \mathrm{dd}, J=8.5$, $3.0 \mathrm{~Hz}), 4.67(1 \mathrm{H}, \mathrm{d}, J=8.5 \mathrm{~Hz}), 3.34-3.27(2 \mathrm{H}, \mathrm{m}), 3.20-3.08(2 \mathrm{H}, \mathrm{m}), 2.26(6 \mathrm{H}, \mathrm{s}), 2.26(6 \mathrm{H}$, s), 2.25 (6H, s), 2.24 (6H, s), 1.65-1.50 (6H, m), 1.37 (3H, s), 0.25 (3H, s); ${ }^{13} \mathrm{C}$ NMR (100 MHz, $\left.\mathrm{CDCl}_{3}\right) \delta 147.5,147.0,142.1,137.3,136.9,136.7,136.4,129.2,128.9,128.8,128.7,127.1$, 126.8, 125.3, 111.5, 83.3, 82.9, 82.7, 81.4, 81.3, 81.2, 77.4, 45.3, 45.1, 27.9, 27.2, 27.2, 25.7, 25.5, 21.9, 21.8, 21.7; ${ }^{31} \mathrm{P}$ NMR (75 MHz, $\left.\mathrm{CDCl}_{3}\right) \delta 138.76$; HRMS (ESI) m/e calcd $\left(\mathrm{M}+\mathrm{H}^{+}\right)$ 692.3863, found 692.3843. 


\section{I.7 $\quad{ }^{1} \mathrm{H}$ and ${ }^{13} \mathrm{C}$ NMR Spectra for New Ligands}<smiles>COC(C)(C)OC1C(c2ccccc2)P(N(C)C)OC(c2ccccc2)(c2ccccc2)C1c1ccccc1</smiles>
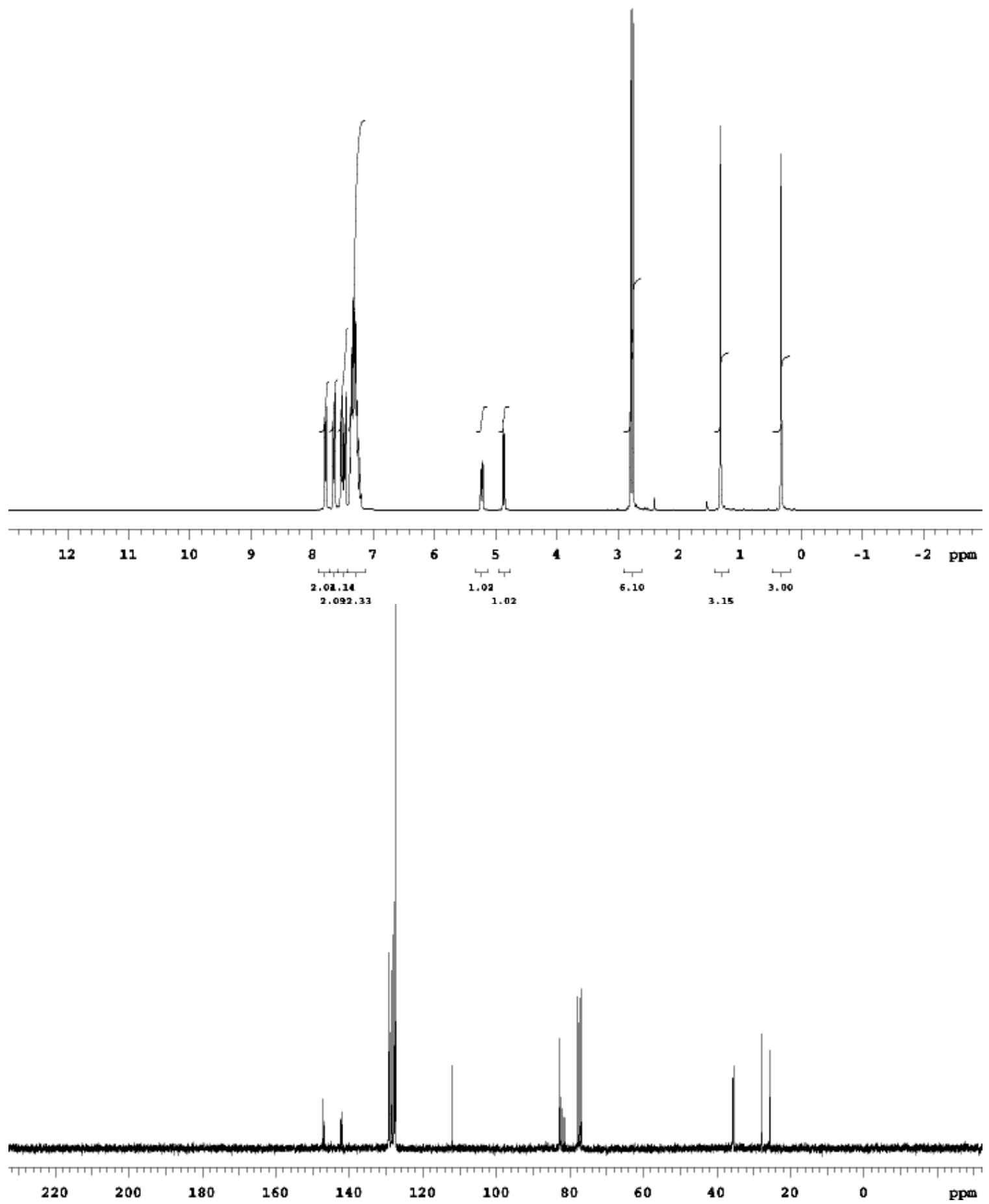
<smiles>Cc1cc(C)cc([As]=[Al][Al])c1</smiles>

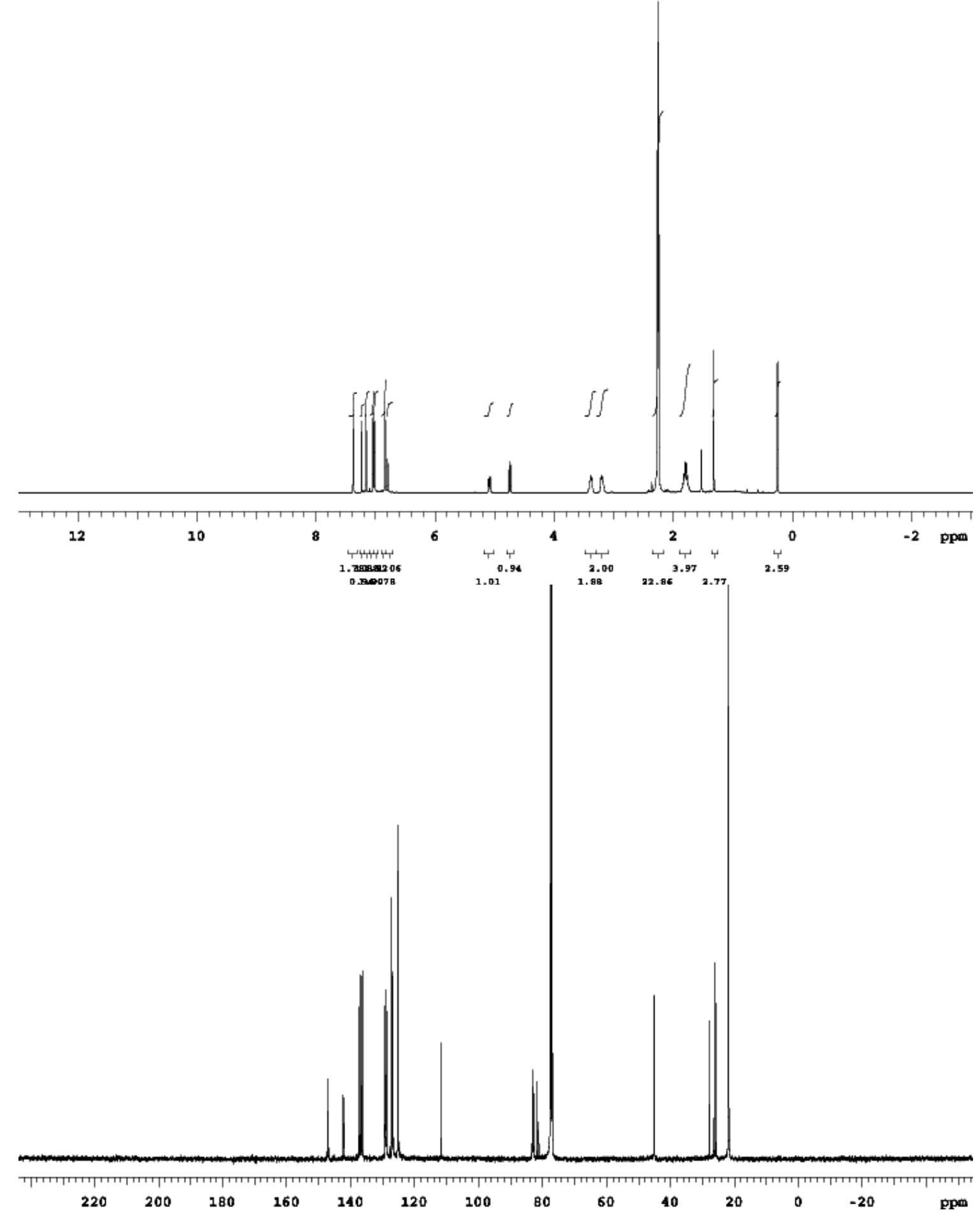




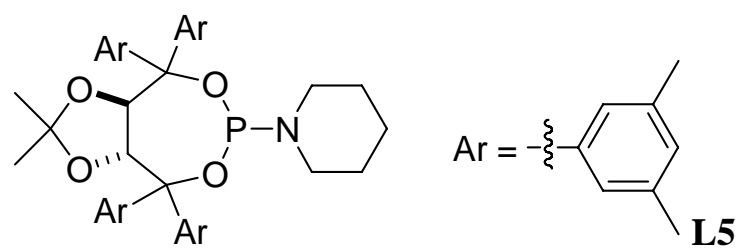
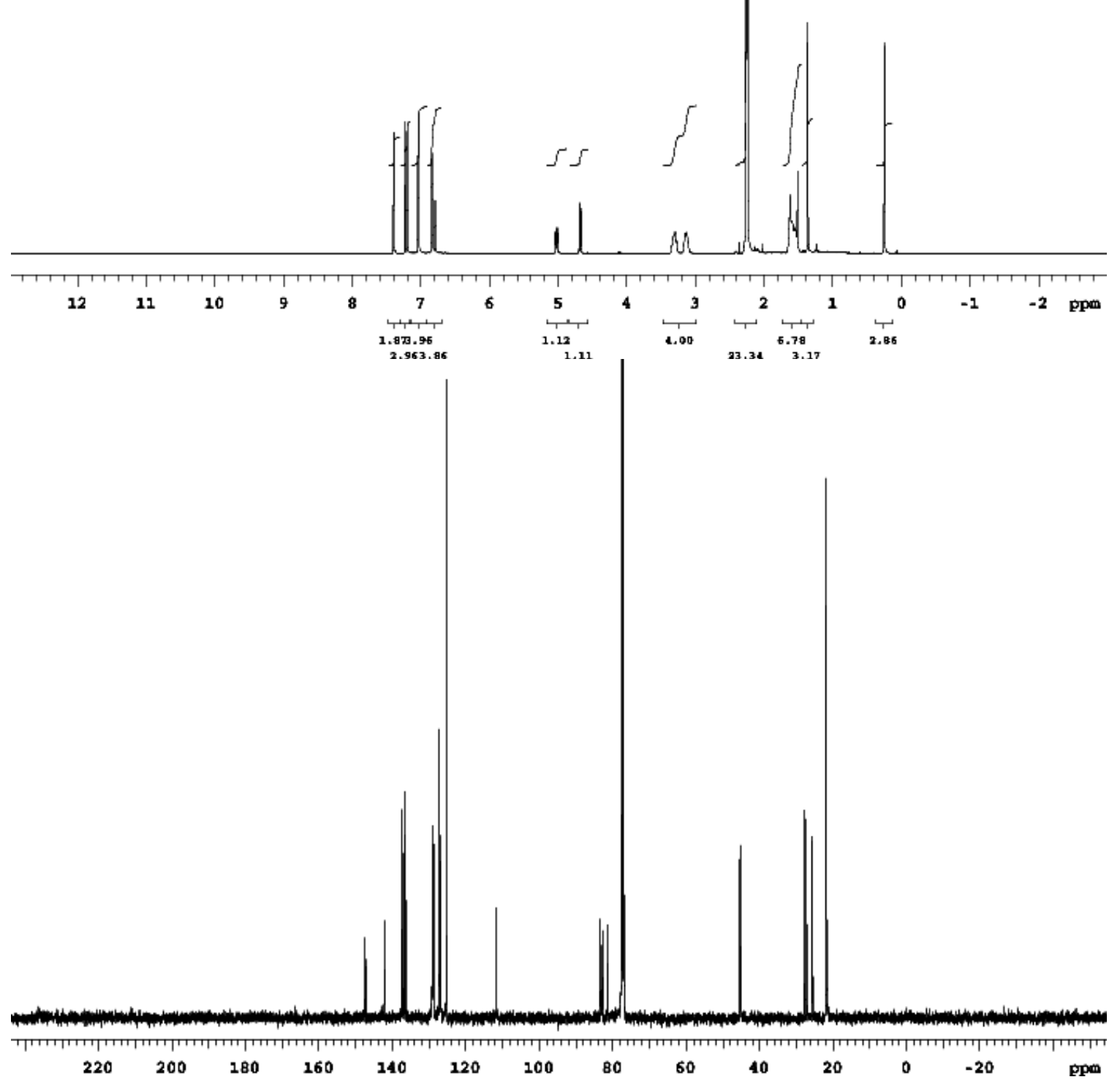


\section{I.8 General procedure for [2+2+2] cycloadditions with Aryl Alkynes.}

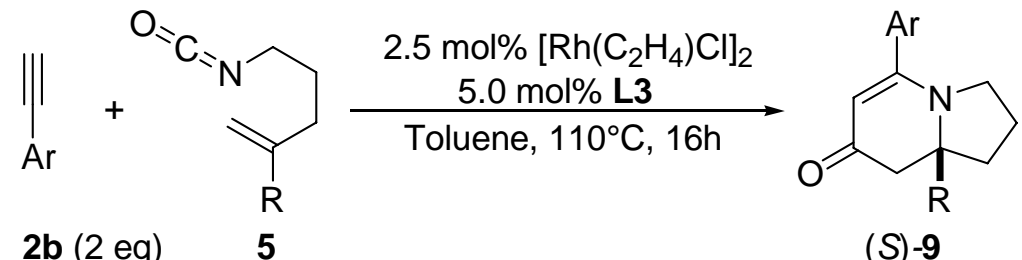

In a glovebox under $\mathrm{N}_{2}$ atmosphere, chlorobis(ethylene)rhodium(I) dimer (2.3 mg, $0.006 \mathrm{mmol}$ ) and dimethyl-TADDOL-phosphoramidite $\mathbf{L 3}$ (6.5 $\mathrm{mg}, 0.012 \mathrm{mmol})$ were transferred into a round bottom flasked fitted with a reflux condenser. The system was sealed with a standard septum, removed from the glovebox and flushed with Ar. A solution of alkyne $(0.48 \mathrm{mmol})$ and isocyanate $(0.24 \mathrm{mmol})$ in toluene $(7 \mathrm{~mL})$ was then added. The brown-black solution was then heated to $110{ }^{\circ} \mathrm{C}$ (bath temperature), stirred at reflux for 16 hours under a static atmosphere of Ar, and cooled. The crude mixture was then concentrated and purified by silica gel, column chromatography.

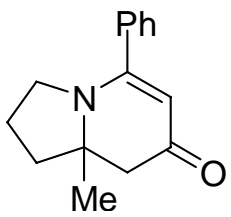

(S)-8a-Methyl-5-phenyl-2,3,8,8a-tetrahydro-1H-indolizin-7-one (7a): Flash Chromatography (Hexanes:EtOAc;1:6) yielded a clear syrup (76\% yield); $91 \%$ ee by HPLC (Chiralcel ODH, Hex:iPrOH;85:15, $\left.\left.1 \mathrm{ml} / \mathrm{min}, \mathrm{RT}_{\text {major }}=20.9 \mathrm{~min}, \mathrm{RT}_{\text {minor }}=15.0 \mathrm{~min}\right) ; \alpha\right]_{\mathrm{D}}=+285.0^{\circ}\left(\mathrm{CHCl}_{3}\right.$, $\mathrm{c}=0.9) ; \mathrm{R}_{f}=0.20$ (100\% EtOAc); IR (Thin Film) v 2962, 2871, 1628, 1533, 1461, 1265, $1119 \mathrm{~cm}^{-}$ ${ }^{1}$; ${ }^{1} \mathrm{H}$ NMR (300 MHz, $\left.\mathrm{CDCl}_{3}\right) \delta$ 7.40-7.25 (5H, m), $5.01(1 \mathrm{H}, \mathrm{s}), 3.59-3.51(1 \mathrm{H}, \mathrm{m}), 3.22-3.13$ $(1 \mathrm{H}, \mathrm{m}), 2.69(1 \mathrm{H}, \mathrm{d}, J=16.5 \mathrm{~Hz}), 2.32(1 \mathrm{H}, \mathrm{d}, J=16.5 \mathrm{~Hz}), 2.10-1.90(4 \mathrm{H}, \mathrm{m}), 1.41(3 \mathrm{H}, \mathrm{s}) ;{ }^{13} \mathrm{C}$ NMR $\left(100 \mathrm{MHz}, \mathrm{CDCl}_{3}\right) \delta 191.8,161.5,136.8,130.1,128.6,127.9,99.3,62.9,50.2,48.0,40.0$, 24.1, 22.0; MS (EI) m/e (rel intensity) 228 (100), 227 (27), 226 (11), 212 (19), 154 (9), 136 (12); HRMS (EI) m/e calcd $\left(\mathrm{M}^{+}\right)$228.1388, found 228.1400.

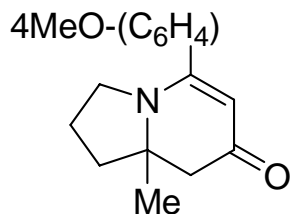

(S)-5-(4-Methoxy-phenyl)-8a-methyl-2,3,8,8a-tetrahydro-1H-indolizin-7-one (7b): Flash Chromatography (Hexanes:EtOAc;1:6) yielded a clear syrup (80\%); 91\% ee by HPLC (Chiralcel $\mathrm{ODH}$, Hex:iPrOH;85:15, $\left.1 \mathrm{ml} / \mathrm{min}, \mathrm{RT}_{\text {major }}=11.2 \mathrm{~min}, \mathrm{RT}_{\text {minor }}=14.9 \mathrm{~min}\right) ;[\alpha]_{\mathrm{D}}=+225.0^{\circ}$ $\left(\mathrm{CHCl}_{3}, \mathrm{c}=0.6\right) ; \mathrm{R}_{f}=0.25$ (100\% EtOAc); IR (Thin Film) v 2964, 1606, 1578, 1508, 1461, 1245, 1174, $1028 \mathrm{~cm}^{-1}$; ${ }^{1} \mathrm{H}$ NMR (400 MHz, $\left.\mathrm{CDCl}_{3}\right) \delta 7.31(2 \mathrm{H}, \mathrm{d}, J=8.5 \mathrm{~Hz}), 6.88(2 \mathrm{H}, \mathrm{d}, J=8.5$ 
Hz), $5.06(1 \mathrm{H}, \mathrm{s}), 3.81(3 \mathrm{H}, \mathrm{s}), 3.56(1 \mathrm{H}, \mathrm{ddd}, J=10.5,6.5,6.5 \mathrm{~Hz}), 3.18(1 \mathrm{H}, \mathrm{m}), 2.65(1 \mathrm{H}, \mathrm{d}, J$ $=16.5 \mathrm{~Hz}), 2.26(1 \mathrm{H}, \mathrm{d}, J=16.5 \mathrm{~Hz}), 2.04-1.85(4 \mathrm{H}, \mathrm{m}), 1.38(3 \mathrm{H}, \mathrm{s}) ;{ }^{13} \mathrm{C}$ NMR $(100 \mathrm{MHz}$, $\left.\mathrm{CDCl}_{3}\right) \delta 192.0,161.6,161.2,129.8,129.2,114.0,99.0,62.7,55.6,50.6,47.8,39.9,24.2$, 22.3; MS (EI) m/e (rel intensity) 258 (100), 257 (34), 242 (14), 154 (10), 136 (7); HRMS (ESI) m/e calcd $\left(\mathrm{M}^{+}\right)$258.1494, found 258.1485 .

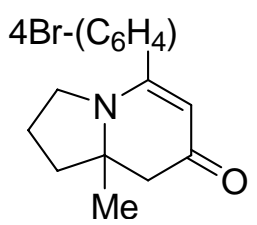

Flash Chromatography (Hex:EtOAc;1:4) yielded an off white solid 6c (19\%) followed by an off white solid 7c (58\%):

(S)-5-(4-Bromo-phenyl)-8a-methyl-2,3,8,8a-tetrahydro-1H-indolizin-7-one (7c): $88 \%$ ee by HPLC (Chiralcel ODH, Hex:iPrOH;85:15, $1 \mathrm{ml} / \mathrm{min}, \mathrm{RT}_{\text {major }}=10.1 \mathrm{~min}, \mathrm{RT}_{\text {minor }}=14.5 \mathrm{~min}$ ); $[\alpha]_{\mathrm{D}}=[\alpha]_{\mathrm{D}}=+153.0^{\circ}\left(\mathrm{CHCl}_{3}, \mathrm{c}=0.5\right) ; \mathrm{R}_{f}=0.35$ (EtOAc 100\%); IR (Thin Film) $v 2929,2858$, 1625, 1526, 1445, 1262, $1212 \mathrm{~cm}^{-1} ;{ }^{1} \mathrm{H}$ NMR (400 MHz, $\left.\mathrm{CDCl}_{3}\right) \delta 7.52$ (2H, d, J= $\left.8.5 \mathrm{~Hz}\right), 7.24$ $(2 \mathrm{H}, \mathrm{d}, J=8.5 \mathrm{~Hz}), 5.04(1 \mathrm{H}, \mathrm{s}), 3.53-3.47(1 \mathrm{H}, \mathrm{m}), 3.17-3.10(1 \mathrm{H}, \mathrm{m}), 2.65(1 \mathrm{H}, \mathrm{d}, J=16.0 \mathrm{~Hz})$, $2.30(1 \mathrm{H}, \mathrm{d}, J=16.0 \mathrm{~Hz}), 2.03-1.89(4 \mathrm{H}, \mathrm{m}), 1.37(3 \mathrm{H}, \mathrm{s}) ;{ }^{13} \mathrm{C}$ NMR $\left(100 \mathrm{MHz}, \mathrm{CDCl}_{3}\right) \delta 191.9$, 160.5, 135.7, 132.1, 132.0, 129.7, 127.6, 124.6, 99.4, 63.0, 50.2, 47.7, 39.9, 24.0, 22.0; MS (FAB) m/e (rel intensity) 308 (20), 306 (22), 221 (18), 207 (17), 154 (16), 147 (29), 136 (23), 133 (100); HRMS (FAB) m/e calcd $\left(\mathrm{M}^{+}\right)$306.0494, found 306.0487.

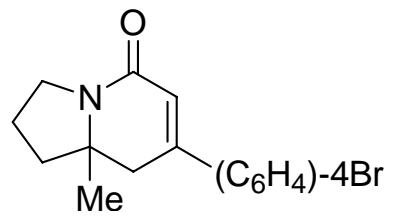

(R)-7-(4-Bromo-phenyl)-8a-methyl-2,3,8,8a-tetrahydro-1H-indolizin-5-one (6c): $82 \%$ ee by HPLC (Chiralcel ODH, Hex:iPrOH;85:15, $1 \mathrm{ml} / \mathrm{min}, \mathrm{RT}_{\text {major }}=7.5 \mathrm{~min}, \mathrm{RT}_{\text {minor }}=8.2 \mathrm{~min}$ ); $[\alpha]_{\mathrm{D}}$ $=+107.0^{\circ}\left(\mathrm{CHCl}_{3}, \mathrm{c}=0.3\right) ; \mathrm{R}_{f}=0.45$ (EtOAc 100\%); IR (Thin Film) v 2967, 2882, 1645, 1595, 1459, $1434 \mathrm{~cm}^{-1} ;{ }^{1} \mathrm{H}$ NMR (400 MHz, $\left.\mathrm{CDCl}_{3}\right) \delta 7.49(2 \mathrm{H}, \mathrm{d}, J=8.5 \mathrm{~Hz}), 7.32(2 \mathrm{H}$, d, $J=8.5 \mathrm{~Hz}), 6.26(1 \mathrm{H}, \mathrm{d}, J=1.5 \mathrm{~Hz}), 3.66-3.54(2 \mathrm{H}, \mathrm{m}), 2.77(1 \mathrm{H}, \mathrm{d}, J=16.5 \mathrm{~Hz})$, $2.71(1 \mathrm{H}, \mathrm{dd}, J=16.5,1.5 \mathrm{~Hz}), 2.10-1.70(4 \mathrm{H}, \mathrm{m}), 1.22(3 \mathrm{H}, \mathrm{s}) ;{ }^{13} \mathrm{C}$ NMR $(100 \mathrm{MHz}$, $\left.\mathrm{CDCl}_{3}\right) \delta 163.1,146.5,137.4,132.1,127.6,123.7,120.4,61.0,43.9,41.0,40.2,23.7$, 21.6.

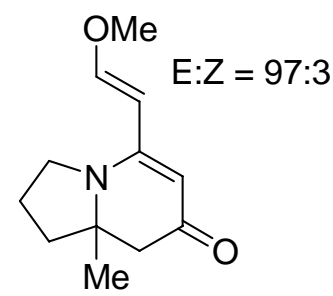

(S)-5-(2-Methoxy-vinyl)-8a-methyl-2,3,8,8a-tetrahydro-1H-indolizin-7-one (7d): Flash Chromatography (Hex:EtOAc;1:6) yielded a yellow syrup (58\%); 91\% ee by HPLC (Chiralcel ODH, Hex:iPrOH;80:20, $\left.1 \mathrm{ml} / \mathrm{min}, \mathrm{RT}_{\text {major }}=9.3 \mathrm{~min}, \mathrm{RT}_{\text {minor }}=14.4 \mathrm{~min}\right) ;:[\alpha]_{\mathrm{D}}=+342.0^{\circ}$ $\left(\mathrm{CHCl}_{3}, \mathrm{c}=1.3\right.$ ); $\mathrm{R}_{f}=0.25$ (EtOAc 100\%); IR (Thin Film) v 2965, 2871, 1627, 1530, 1495, 1277, 
$1009 \mathrm{~cm}^{-1}$; ${ }^{1} \mathrm{H}$ NMR $\left(400 \mathrm{MHz}, \mathrm{CDCl}_{3}\right) \delta 7.11(1 \mathrm{H}, \mathrm{d}, J=12.5 \mathrm{~Hz}), 5.26(1 \mathrm{H}, \mathrm{d}, J=12.5 \mathrm{~Hz})$, $5.03(1 \mathrm{H}, \mathrm{s}), 3.65(3 \mathrm{H}, \mathrm{s}), 3.62-3.55(1 \mathrm{H}, \mathrm{m}), 3.43-3.35(1 \mathrm{H}, \mathrm{m}), 2.50(1 \mathrm{H}, \mathrm{d}, J=16.0 \mathrm{~Hz}), 2.26$ $(1 \mathrm{H}, \mathrm{d}, J=16.0 \mathrm{~Hz}), 2.05-1.93(3 \mathrm{H}, \mathrm{m}), 1.84-1.75(1 \mathrm{H}, \mathrm{m}), 1.21(3 \mathrm{H}, \mathrm{s}) ;{ }^{13} \mathrm{C}$ NMR $(100 \mathrm{MHz}$, $\left.\mathrm{CDCl}_{3}\right) \delta 190.4,157.5,156.8,99.5,91.5,62.6,57.8,48.5,47.7,40.0,22.6,20.5$; MS (FAB) $\mathrm{m} / e$ (rel intensity) 208 (100), 207 (39), 155 (23), 154 (76), 139 (10), 138 (25), 137 (46), 136 (43); HRMS (FAB) $m / e$ calcd $\left(\mathrm{M}+\mathrm{H}^{+}\right)$208.1332, found 208.1337.

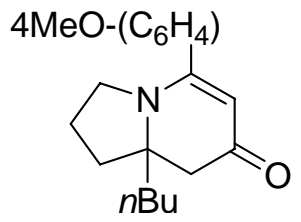

(S)-8a-Butyl-5-(4-methoxy-phenyl)-2,3,8,8a-tetrahydro-1H-indolizin-7-one $\quad$ (9b): Flash Chromatography (Hexanes:EtOAc;1:6) yielded a clear syrup (71\%); 90\% ee by HPLC (Chiralcel ODH, Hex:iPrOH; 80:20, $\left.1 \mathrm{ml} / \mathrm{min}, \mathrm{RT}_{\text {major }}=6.6 \mathrm{~min}, \mathrm{RT}_{\text {minor }}=9.5 \mathrm{~min}\right) ;[\alpha]_{\mathrm{D}}=305.0^{\circ}\left(\mathrm{CHCl}_{3}\right.$, c=0.8); $\mathrm{R}_{f}=0.35$ (100\% EtOAc); IR (Thin Film) v 2956, 1605, 1626, 1578, 1508, 1453, 1243, $1173 \mathrm{~cm}^{-1}$; ${ }^{1} \mathrm{H}$ NMR (400 MHz, $\left.\mathrm{CDCl}_{3}\right) \delta 7.34(2 \mathrm{H}, \mathrm{d}, J=8.0 \mathrm{~Hz}), 6.88(2 \mathrm{H}, \mathrm{d}, J=8.0 \mathrm{~Hz}), 5.07$ (1H, s), 3.81 (3H, s), 3.48 (1H, ddd, $J=11.0,7.5,7.5 \mathrm{~Hz}), 3.23(1 \mathrm{H}$, ddd, $J=11.0,5.0,5.0 \mathrm{~Hz}$ ), $2.62(1 \mathrm{H}, \mathrm{d}, J=16.5 \mathrm{~Hz}), 2.31(1 \mathrm{H}, \mathrm{d}, J=16.5 \mathrm{~Hz}), 2.11-2.04(1 \mathrm{H}, \mathrm{m}), 1.90-1.72$ (5H, m), 1.44$1.16(4 \mathrm{H}, \mathrm{m}), 0.87$ (3H, t, $J=7.0 \mathrm{~Hz}) ;{ }^{13} \mathrm{C}$ NMR $\left(100 \mathrm{MHz}, \mathrm{CDCl}_{3}\right) \delta 192.2,161.9,161.4,129.9$, 129.4, 114.1, 99.1, 65.5, 55.6, 52.1, 45.8, 36.8, 34.2, 26.6, 25.0, 23.3, 14.3; HRMS (ESI) m/e calcd $\left(\mathrm{M}^{+}\right)$300.1958, found 300.1960.

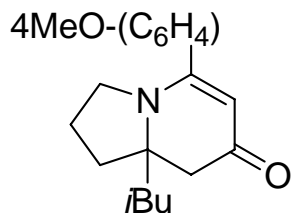

(S)-8a-Isobutyl-5-(4-methoxy-phenyl)-2,3,8,8a-tetrahydro-1H-indolizin-7-one (9c): Flash Chromatography (Hexanes:EtOAc;1:6) yielded a clear syrup (75\%); 94\% ee by HPLC (Chiralcel ODH, Hex:iPrOH; 80:20, $\left.1 \mathrm{ml} / \mathrm{min}, \mathrm{RT}_{\text {major }}=6.7 \mathrm{~min}, \mathrm{RT}_{\text {minor }}=10.4 \mathrm{~min}\right) ;[\alpha]_{\mathrm{D}}=314.0^{\circ}\left(\mathrm{CHCl}_{3}\right.$, $\mathrm{c}=1.0) ; \mathrm{R}_{f}=0.33$ (100\% EtOAc); IR (Thin Film) v 2955, 1606, 1627, 1508, 1456, 1246, $1174 \mathrm{~cm}^{-}$ ; ${ }^{1} \mathrm{H}$ NMR (400 MHz, CDCl $\left.{ }_{3}\right) \delta 7.34(2 \mathrm{H}, \mathrm{d}, J=7.0 \mathrm{~Hz}), 6.88(2 \mathrm{H}, \mathrm{d}, J=7.0 \mathrm{~Hz}), 5.11(1 \mathrm{H}, \mathrm{s})$, 3.81 (3H, s), 3.52 (1H, ddd, $J=11.0,7.5,7.5 \mathrm{~Hz}), 3.23(1 \mathrm{H}, \mathrm{ddd}, J=11.0,5.5,5.5 \mathrm{~Hz}), 2.62(1 \mathrm{H}$, d, $J=16.0 \mathrm{~Hz}), 2.31(1 \mathrm{H}, \mathrm{d}, J=16.0 \mathrm{~Hz}), 2.15-2.08(1 \mathrm{H}, \mathrm{m}), 1.91-1.81(4 \mathrm{H}, \mathrm{m}), 1.77-1.68(1 \mathrm{H}$, m), $1.60(1 \mathrm{H}, \mathrm{dd}, J=14.0,7.0 \mathrm{~Hz}), 0.95(3 \mathrm{H}, \mathrm{d}, J=6.5 \mathrm{~Hz}), 0.93(3 \mathrm{H}, \mathrm{d}, J=6.5 \mathrm{~Hz}) ;{ }^{13} \mathrm{C}$ NMR $\left(100 \mathrm{MHz}, \mathrm{CDCl}_{3}\right) \delta 192.3,161.9,161.4,129.9,129.3,114.1,99.4,65.8,55.6,45.7,42.7,37.7$, 25.4, 25.0, 24.8, 24.3; HRMS (ESI) m/e calcd $\left(\mathrm{M}^{+}\right)$300.1958, found 300.1944.<smiles>CCCCN1CC(C)C=CC1C(C)(C)OC</smiles>

(S)-8a-Benzyl-5-(4-methoxy-phenyl)-2,3,8,8a-tetrahydro-1H-indolizin-7-one (9d): Flash Chromatography (Hex:EtOAc;1:6) yielded a light yellow solid (80\%); 92\% ee by HPLC (Chiralcel ODH, Hex:iPrOH;85:15, $1 \mathrm{ml} / \mathrm{min}, \mathrm{RT}_{\text {major }}=13.4 \mathrm{~min}, \mathrm{RT}_{\text {minor }}=19.5 \mathrm{~min}$ ); $[\alpha]_{\mathrm{D}}=$ $235.0^{\circ}\left(\mathrm{CHCl}_{3}, \mathrm{c}=1.1\right) ; \mathrm{R}_{f}=0.25$ (EtOAc 100\%); IR (Thin Film) v 2962, 1625, 1605, 1508, 1454, 
1245, 1175, $1027 \mathrm{~cm}^{-1} ;{ }^{1} \mathrm{H}$ NMR (400 MHz, $\left.\mathrm{CDCl}_{3}\right) \delta 7.36(2 \mathrm{H}, \mathrm{d}, J=7.0 \mathrm{~Hz}), 7.32-7.23(3 \mathrm{H}$, m), $7.18(2 \mathrm{H}, \mathrm{d}, J=8.0 \mathrm{~Hz}), 6.89(2 \mathrm{H}, \mathrm{d}, J=8.0 \mathrm{~Hz}), 5.25(1 \mathrm{H}, \mathrm{s}), 3.81(3 \mathrm{H}, \mathrm{s}), 3.34(1 \mathrm{H}, \mathrm{d}, J=$ $13.5 \mathrm{~Hz}), 3.13-3.10(1 \mathrm{H}, \mathrm{m}), 3.15-2.97(1 \mathrm{H}, \mathrm{m}), 2.78(1 \mathrm{H}, \mathrm{d}, J=13.0 \mathrm{~Hz}), 2.65(1 \mathrm{H}, \mathrm{d}, J=16.0$ $\mathrm{Hz}), 2.34(1 \mathrm{H}, \mathrm{d}, J=16.0 \mathrm{~Hz}), 2.30-2.22(1 \mathrm{H}, \mathrm{m}), 1.78-1.69(2 \mathrm{H}, \mathrm{m}), 1.65-1.60(1 \mathrm{H}, \mathrm{m}) ;{ }^{13} \mathrm{C}$ NMR $\left(100 \mathrm{MHz}, \mathrm{CDCl}_{3}\right) \delta 192.2,161.8,161.6,137.1,131.2,130.2,128.9,128.3,126.9,114.1$,

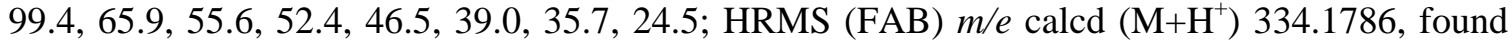
334.1795.

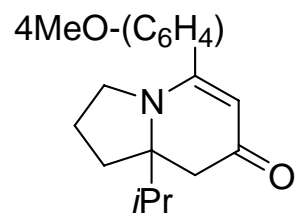

(S)-8a-Isopropyl-5-(4-methoxy-phenyl)-2,3,8,8a-tetrahydro-1H-indolizin-7-one (9e): Flash Chromatography (Hexanes:EtOAc;1:3) yielded a yellow syrup (50\%); 91\% ee by HPLC (Chiralcel ODH, Hex:iPrOH; 85:15, $1 \mathrm{ml} / \mathrm{min}, \mathrm{RT}_{\text {major }}=8.8 \mathrm{~min}, \mathrm{RT}_{\text {minor }}=10.3 \mathrm{~min}$ ); $[\alpha]_{\mathrm{D}}=$ $+320.0^{\circ}\left(\mathrm{CHCl}_{3}, \mathrm{c}=0.9\right) ; \mathrm{R}_{f}=0.40$ (100\% EtOAc); IR (Thin Film) v 2966, 2874, 1627, 1606, 1508, 1453, $1243 \mathrm{~cm}^{-1} ;{ }^{1} \mathrm{H}$ NMR $\left(400 \mathrm{MHz}, \mathrm{CDCl}_{3}\right) \delta 7.37(2 \mathrm{H}, \mathrm{d}, J=8.0 \mathrm{~Hz}), 6.88(2 \mathrm{H}, \mathrm{d}, J=$ $8.0 \mathrm{~Hz}$ ), $5.14(1 \mathrm{H}, \mathrm{s}), 3.81(3 \mathrm{H}, \mathrm{s}), 3.41(1 \mathrm{H}, \mathrm{ddd}, J=10.5,8.5,8.0 \mathrm{~Hz}), 3.31$ (1H, ddd, $J=10.5$, 5.5, $3.5 \mathrm{~Hz}), 2.65(1 \mathrm{H}$, sept, $J=7.0 \mathrm{~Hz}), 2.64(1 \mathrm{H}, \mathrm{d}, J=16.5 \mathrm{~Hz}), 2.50(1 \mathrm{H}, \mathrm{d}, J=16.5 \mathrm{~Hz})$, 2.12-2.01 (1H, m), 1.90-1.82 (2H, m), 1.64-1.58 (1H, m), 0.98 (3H, d, $J=7.0 \mathrm{~Hz}), 0.90$ (3H, d, $J$ $=7.0 \mathrm{~Hz}) ;{ }^{13} \mathrm{C}$ NMR $\left(100 \mathrm{MHz}, \mathrm{CDCl}_{3}\right) \delta 192.1,162.4,161.6,130.1,129.4,114.1,99.3,69.0$, 55.6, 54.0, 44.1, 31.5, 28.7, 25.5, 17.6, 17.5; MS (EI) m/e (rel intensity) 286 (100), 285 (15), 242 (48); HRMS (ESI) $m / e$ calcd $\left(\mathrm{M}^{+}\right)$286.1807, found 286.1808.

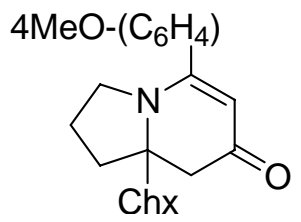

8a-Cyclohexyl-5-(4-methoxy-phenyl)-2,3,8,8a-tetrahydro-1H-indolizin-7-one (9f): Flash Chromatography (Hexanes:EtOAc;1:4) yielded a light yellow syrup (19\%); 87\% ee by HPLC (Chiralcel ODH, Hex:iPrOH; 85:15, $1 \mathrm{ml} / \mathrm{min}, \mathrm{RT}_{\text {major }}=8.6 \mathrm{~min}, \mathrm{RT}_{\text {minor }}=15.9 \mathrm{~min}$ ); $[\alpha]_{\mathrm{D}}=$ 206.0 ${ }^{\circ}\left(\mathrm{CHCl}_{3}, \mathrm{c}=0.8\right) ; \mathrm{R}_{f}=0.25$ (EtOAc 100\%); IR (Thin Film) v 2928, 2851, 1605, 1577, 1535, 1507, 1452, 1247, $1174 \mathrm{~cm}^{-1}$; ${ }^{1} \mathrm{H}$ NMR (400 MHz, $\left.\mathrm{CDCl}_{3}\right) \delta 7.38(2 \mathrm{H}, \mathrm{d}, J=8.5 \mathrm{~Hz}), 6.89(2 \mathrm{H}$, d, $J=8.5 \mathrm{~Hz}), 5.10(1 \mathrm{H}, \mathrm{s}), 3.82(3 \mathrm{H}, \mathrm{s}), 3.37(1 \mathrm{H}, \mathrm{ddd}, J=10.0,8.0,8.0 \mathrm{~Hz}), 3.28(1 \mathrm{H}, \mathrm{m}, J=$ 10.0, 3.5, 3.5 Hz), $2.60(1 \mathrm{H}, \mathrm{d}, J=16.5 \mathrm{~Hz}), 2.51(1 \mathrm{H}, \mathrm{d}, J=16.5 \mathrm{~Hz}), 2.28-2.20(1 \mathrm{H}, \mathrm{m}), 2.11$ (1H, ddd, $J=12.0,9.0,9.0 \mathrm{~Hz}), 1.90-1.56(8 \mathrm{H}, \mathrm{m}), 1.25-0.90(5 \mathrm{H}, \mathrm{m}) ;{ }^{13} \mathrm{C}$ NMR $(100 \mathrm{MHz}$, $\left.\mathrm{CDCl}_{3}\right) \delta 192.3,162.3,161.6,130.2$, 129.5, 114.1, 99.2, 68.6, 55.6, 53.9, 43.9, 39.4, 33.1, 27.6, 27.3, 26.7, 26.6, 26.5, 25.7; HRMS (ESI) m/e calcd $\left(\mathrm{M}^{+}\right)$326.2115, found 326.2100.

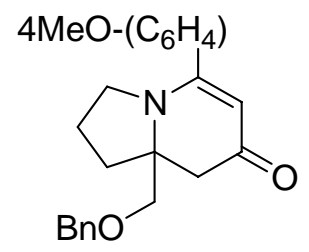

(S)-8a-Benzyloxymethyl-5-(4-methoxy-phenyl)-2,3,8,8a-tetrahydro-1H-indolizin-7-one (9g): Flash Chromatography (Hex:EtOAc;1:6) yielded a yellow syrup (77\%); 92\% ee by HPLC 
(Chiralcel ODH, Hex:iPrOH; 80:20, $1 \mathrm{ml} / \mathrm{min}, \mathrm{RT}_{\text {major }}=11.3 \mathrm{~min}, \mathrm{RT}_{\text {minor }}=14.1 \mathrm{~min}$ ); $[\alpha]_{\mathrm{D}}=$ $165.0^{\circ}\left(\mathrm{CHCl}_{3}, \mathrm{c}=1.0\right) ; \mathrm{R}_{f}=0.26$ (EtOAc 100\%); IR (Thin Film) v 2965, 2870, 1626, 1606, 1509, 1453, 1246, 1175, 1106, $1028 \mathrm{~cm}^{-1}$; ${ }^{1} \mathrm{H}$ NMR (400 MHz, $\left.\mathrm{CDCl}_{3}\right) \delta$ 7.33-7.24 (7H, m), $6.87(2 \mathrm{H}$, d, $J=8.0 \mathrm{~Hz}), 5.05(1 \mathrm{H}), 4.54(1 \mathrm{H}, \mathrm{d}, J=12.0 \mathrm{~Hz}), 4.50(1 \mathrm{H}, \mathrm{d}, J=12.0 \mathrm{~Hz}), 3.82(1 \mathrm{H}, \mathrm{d}, J=$ $10.0 \mathrm{~Hz}), 3.80(3 \mathrm{H}, \mathrm{s}), 3.60-3.52(1 \mathrm{H}, \mathrm{m}), 3.47(1 \mathrm{H}, \mathrm{d}, J=10.0 \mathrm{~Hz}), 3.23-3.17(1 \mathrm{H}, \mathrm{m}), 2.63(1 \mathrm{H}$, d, $J=16.0 \mathrm{~Hz}), 2.45(1 \mathrm{H}, \mathrm{d}, J=16.0 \mathrm{~Hz}), 2.44-2.35(1 \mathrm{H}, \mathrm{m}), 2.00-1.75(3 \mathrm{H}, \mathrm{m}) ;{ }^{13} \mathrm{C}$ NMR $(100$ $\left.\mathrm{MHz} \mathrm{CDCl}_{3}\right) \delta 191.5,162.1,161.4,138.4,130.0,129.1,128.6,127.8,127.6,114.0,99.0,73.7$, 70.3, 65.5, 55.6, 52.2, 43.6, 35.5, 24.6; HRMS (FAB) m/e calcd $\left(\mathrm{M}+\mathrm{H}^{+}\right)$364.1907, found 364.1907.

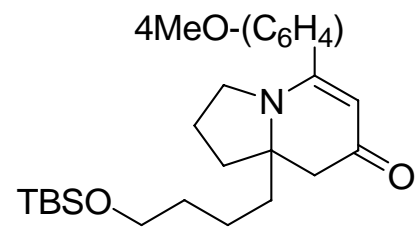

(S)-8a-[4-(tert-Butyl-dimethyl-silanyloxy)-butyl]-5-(4-methoxy-phenyl)-2,3,8,8a-tetrahydro1H-indolizin-7-one (9h): Flash Chromatography (Hexanes:EtOAc;1:6) yielded a clear syrup (74\%); 88\% ee by HPLC (Chiralcel ADH, Hex: $\mathrm{PrOH} ; 90: 10,1 \mathrm{ml} / \mathrm{min}, \mathrm{RT}_{\text {major }}=8.3 \mathrm{~min}$, $\left.\mathrm{RT}_{\text {minor }}=7.8 \mathrm{~min}\right) ;[\alpha]_{\mathrm{D}}=+138.0^{\circ}\left(\mathrm{CHCl}_{3}, \mathrm{c}=0.9\right) ; \mathrm{R}_{f}=0.23(100 \%$ EtOAc); IR (Thin Film) $v$ 2933, 1605, 1507, 1461, 1246, 1174, $1026 \mathrm{~cm}^{-1}$; ${ }^{1} \mathrm{H}$ NMR $\left(300 \mathrm{MHz}, \mathrm{CDCl}_{3}\right) \delta 7.37$ (2H, d, $J=$ $8.5 \mathrm{~Hz}), 6.90(2 \mathrm{H}, \mathrm{d}, J=8.5 \mathrm{~Hz}), 5.12(1 \mathrm{H}, \mathrm{s}), 3.84(3 \mathrm{H}, \mathrm{s}), 3.66-3.58(2 \mathrm{H}, \mathrm{m}), 3.56-3.46(1 \mathrm{H}$, m), $3.27(1 \mathrm{H}$, ddd, $J=11.0,5.0,5.0 \mathrm{~Hz}), 2.68(1 \mathrm{H}, \mathrm{d}, J=16.5 \mathrm{~Hz}), 2.35(1 \mathrm{H}, \mathrm{d}, J=16.5 \mathrm{~Hz})$, 2.20-2.04 (1H, m), 1.95-1.76 (5H, m), 1.60-1.35 (4H, m), $0.89(9 \mathrm{H}, \mathrm{s}), 0.04(6 \mathrm{H}, \mathrm{s}) ;{ }^{13} \mathrm{C}$ NMR $\left(100 \mathrm{MHz}, \mathrm{CDCl}_{3}\right) \delta 193.2,162.2,161.5,130.0,129.3,114.1,99.2,65.6,63.1,55.6,52.4,45.9$, 36.7, 34.3, 33.3, 26.2, 25.0, 20.9, 18.6, -5.0; MS (EI) $\mathrm{m} / e$ (rel intensity) 431 (32), 430 (100), 428 (13), 372 (18), 243 (21), 242 (53); HRMS (EI) m/e calcd ( $\left.\mathrm{M}^{+}\right)$430.2777, found 430.2765.

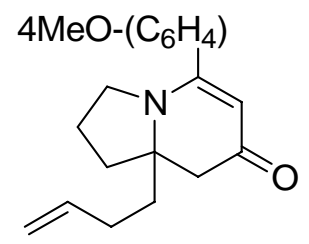

(S)-8a-But-3-enyl-5-(4-methoxy-phenyl)-2,3,8,8a-tetrahydro-1H-indolizin-7-one (9i): Flash Chromatography (Hexanes:EtOAc;1:6) yielded a clear syrup (75\%); 91\% ee by HPLC (Chiralcel $\mathrm{ODH}$, Hex:iPrOH;85:15, $\left.1 \mathrm{ml} / \mathrm{min}, \mathrm{RT}_{\text {major }}=9.6 \mathrm{~min}, \mathrm{RT}_{\text {minor }}=15.5 \mathrm{~min}\right) ;[\alpha]_{\mathrm{D}}=+288.0^{\circ}$ $\left(\mathrm{CHCl}_{3}, \mathrm{c}=1.0\right) ; \mathrm{R}_{f}=0.20$ (100\% EtOAc); IR (Thin Film) v 2969, 2872, 1605, 1578, 1537, 1508, 1454, 1244, 1175, 1028, $913 \mathrm{~cm}^{-1}$; ${ }^{1} \mathrm{H}$ NMR (400 MHz, $\left.\mathrm{CDCl}_{3}\right) \delta 7.33(2 \mathrm{H}, \mathrm{d}, J=8.0 \mathrm{~Hz}), 6.87$ $(2 \mathrm{H}, \mathrm{d}, J=8.0 \mathrm{~Hz}), 5.76(1 \mathrm{H}, \mathrm{ddt}, J=17.0,10.5,6.5 \mathrm{~Hz}), 5.09(1 \mathrm{H}, \mathrm{s}), 5.00$ (1H, dd, $J=17.0,1.5$ Hz), 4.92 (1H, dd, $J=10.5,1.0 \mathrm{~Hz}$ ), 3.80 (3H, s), 3.49 (1H, ddd, $J=11.0,7.5,3.5 \mathrm{~Hz}), 3.24(1 \mathrm{H}$, ddd, $J=11.0,5.5,5.5 \mathrm{~Hz}), 2.64(1 \mathrm{H}, \mathrm{d}, J=16.5 \mathrm{~Hz}), 2.31(1 \mathrm{H}, \mathrm{d}, J=16.5 \mathrm{~Hz}), 2.25-2.00(3 \mathrm{H}$, m), 1.94-1.75 (5H, m); ${ }^{13} \mathrm{C}$ NMR (100 MHz, $\left.\mathrm{CDCl}_{3}\right) \delta 200.0,162.1,161.5,138.1,130.0,129.2$, 115.1, 114.1, 99.4, 65.3, 55.6, 52.3, 45.7, 36.8, 33.7, 28.9, 25.0; MS (EI) $\mathrm{m} / \mathrm{e}$ (rel intensity) 298 (100), 297 (23), 243 (20), 242 (18), 154 (22), 137 (13), 136 (13); HRMS (ESI) m/e calcd (M+) 298.1807, found 298.1808 . 


\section{I.9 ${ }^{1} \mathrm{H}$ and ${ }^{13} \mathrm{C}$ NMR Spectra for [2+2+2] Products from Aryl Alkynes}

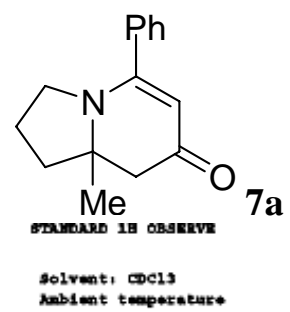

Aablent tompersetar.

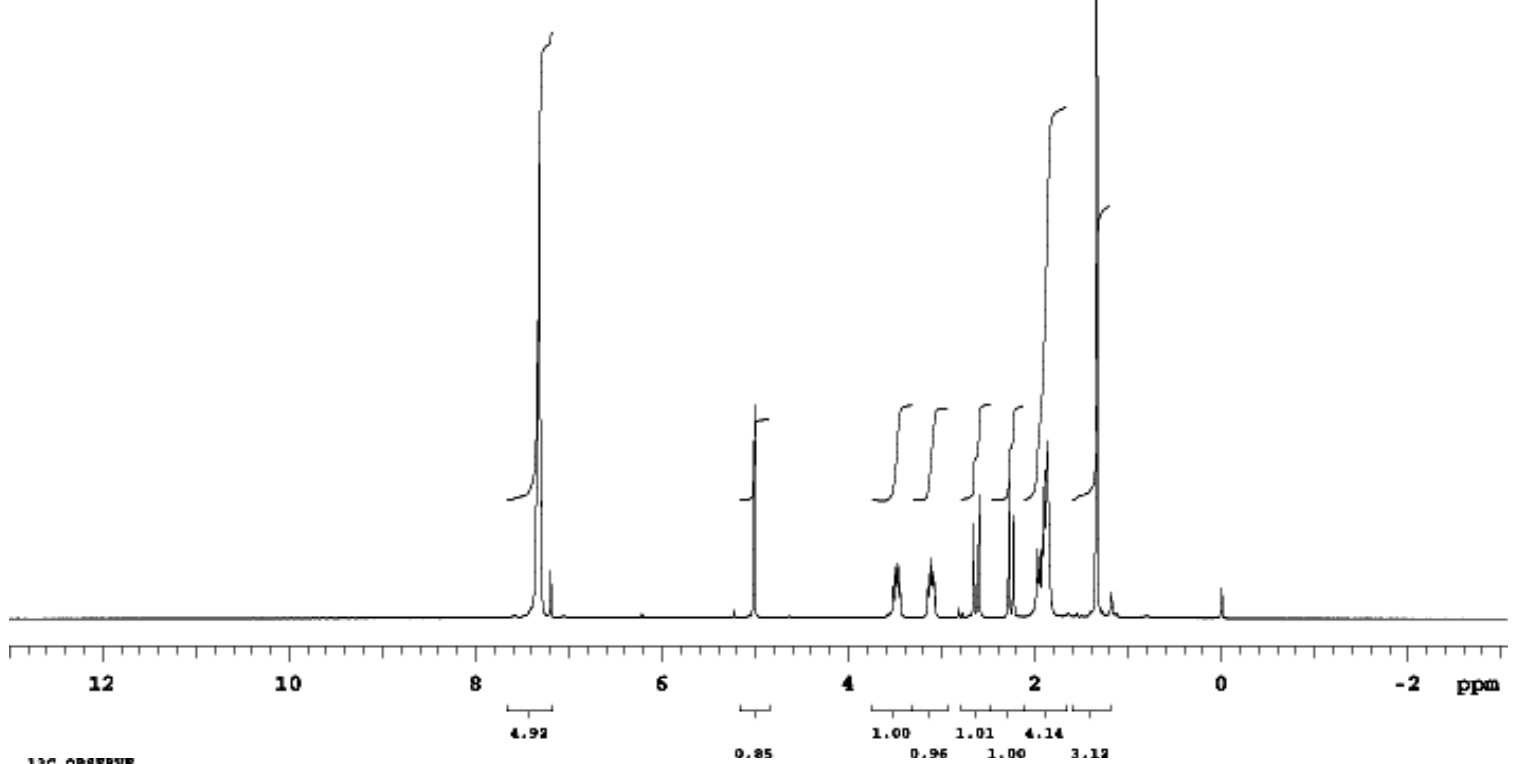

13e OBgrave

-1_1_114n_3

Solvent, CDC13

habient temperakar.

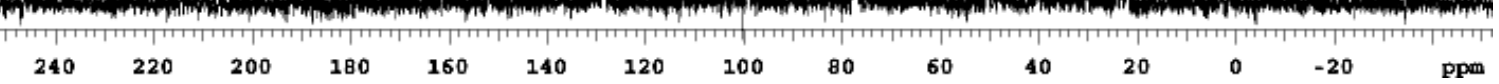



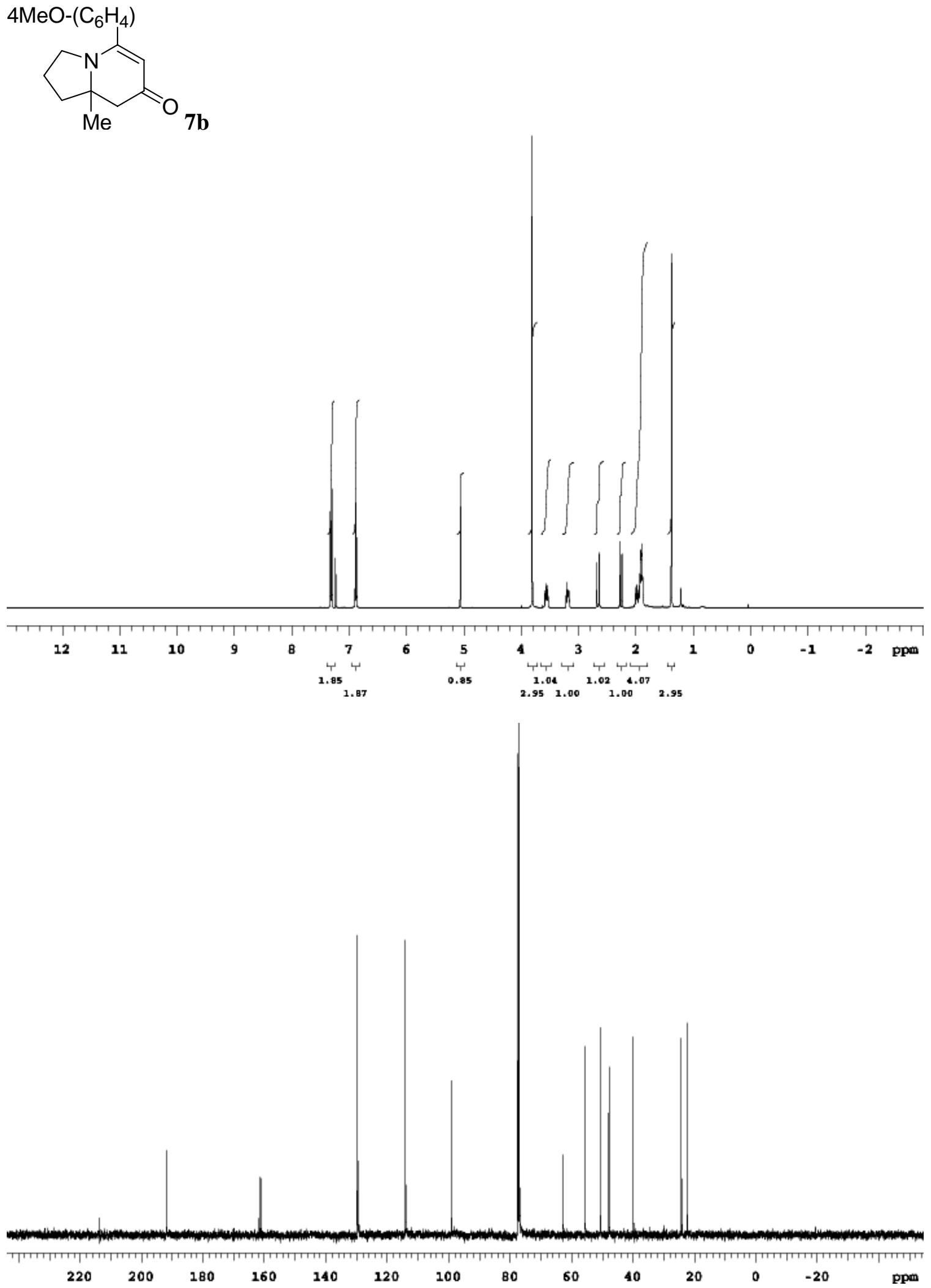

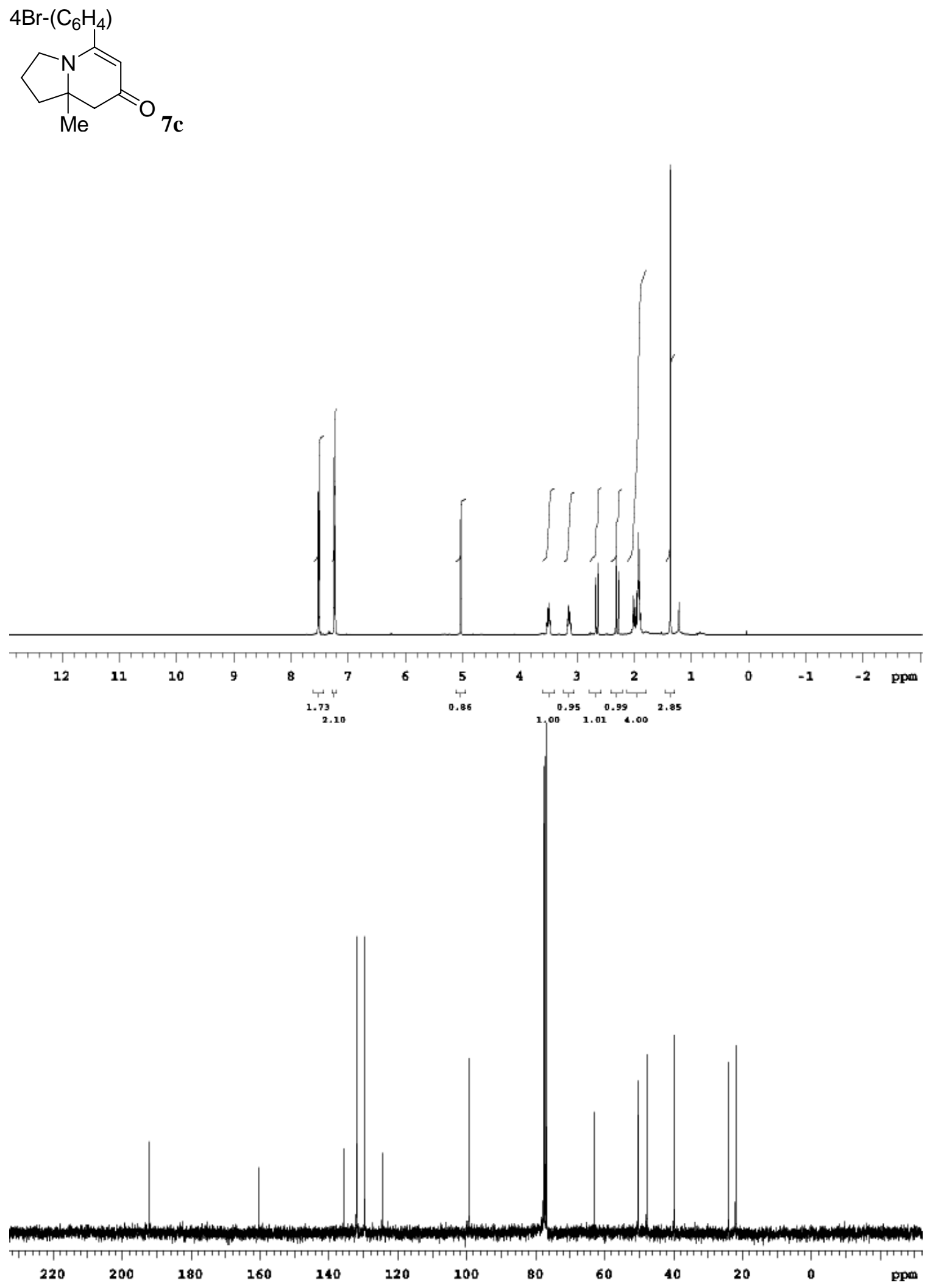
<smiles>CC12CCCN1C(=O)C=C(CCCC(Br)(Br)C(Br)(Br)Br)C2</smiles>

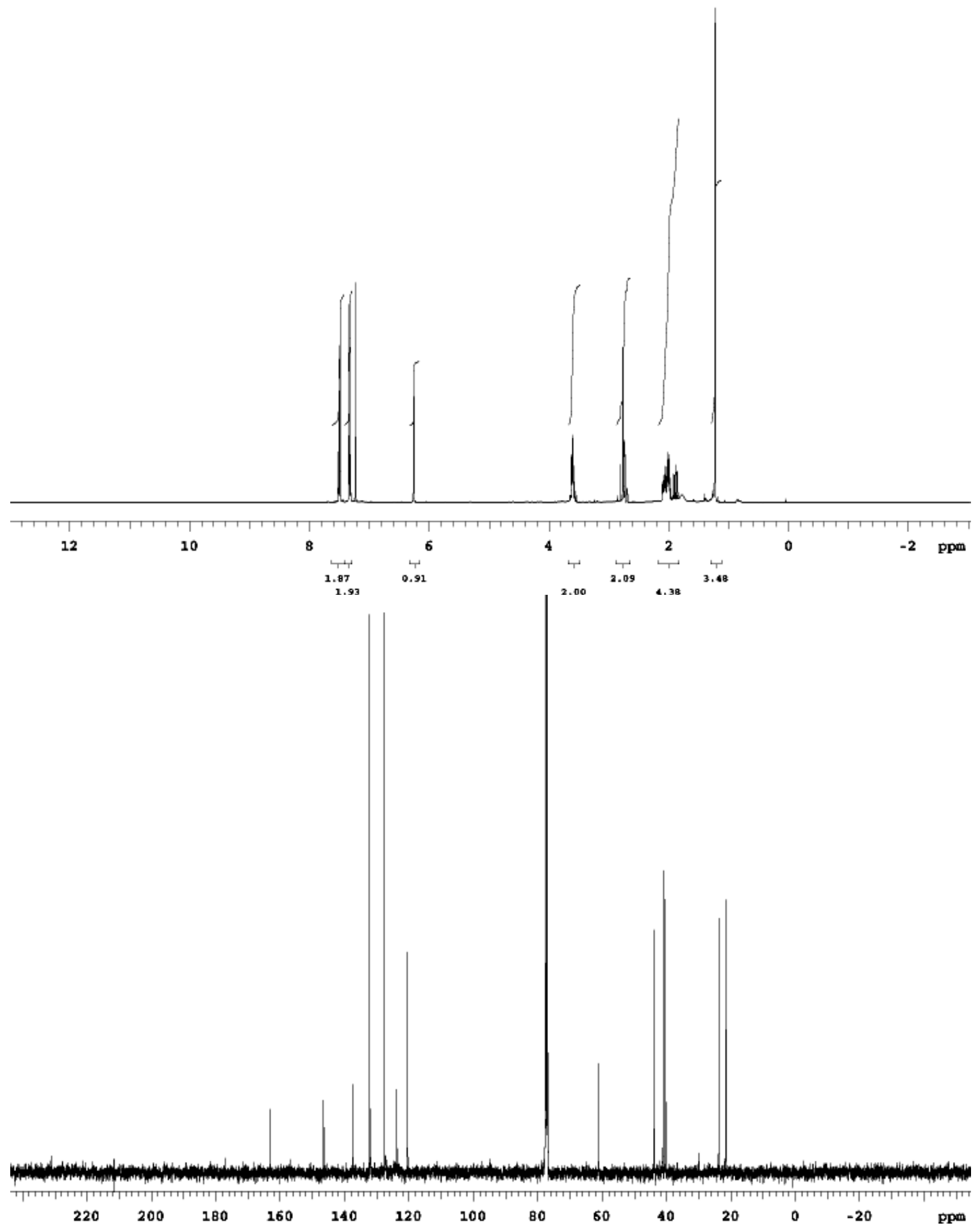



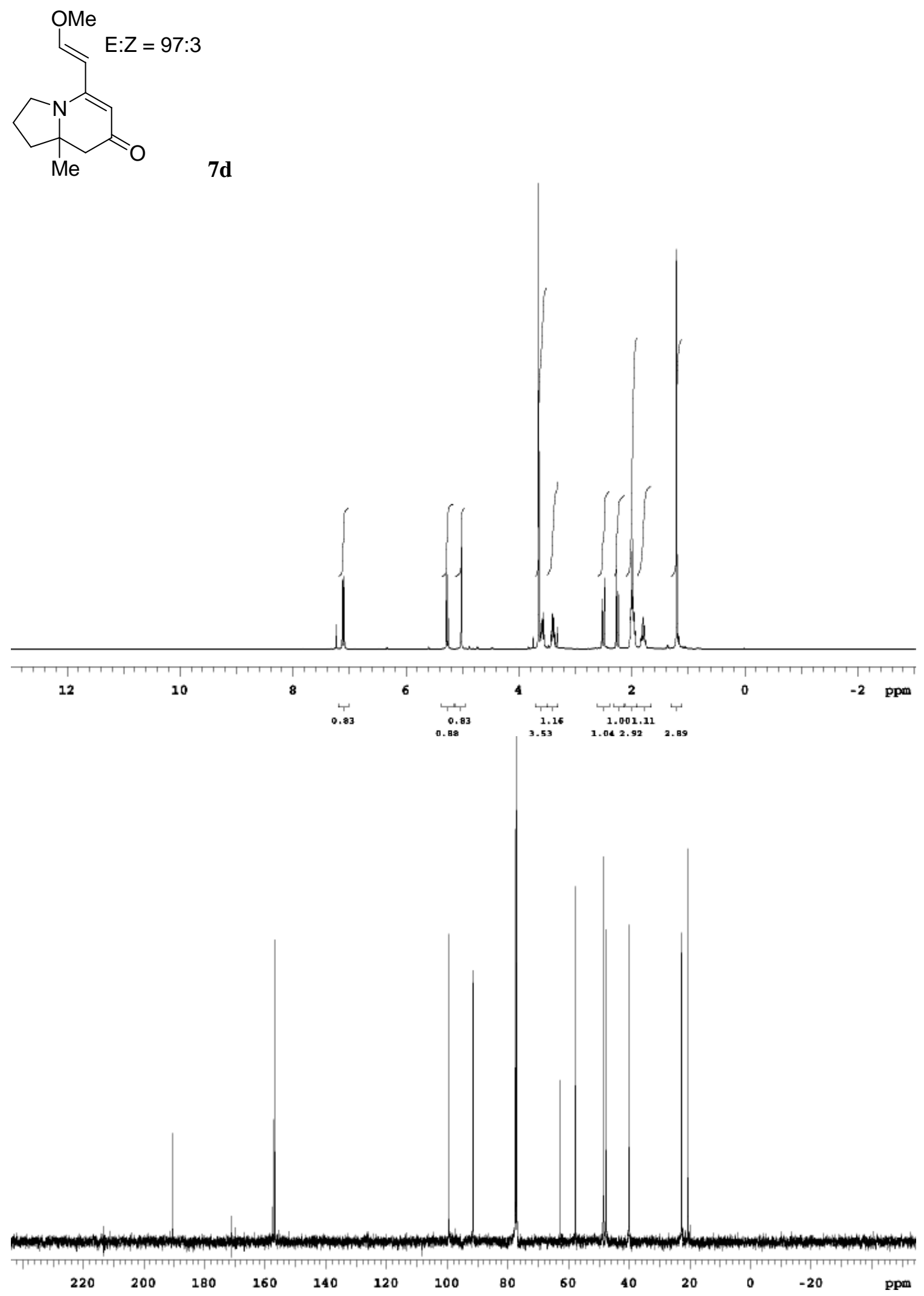

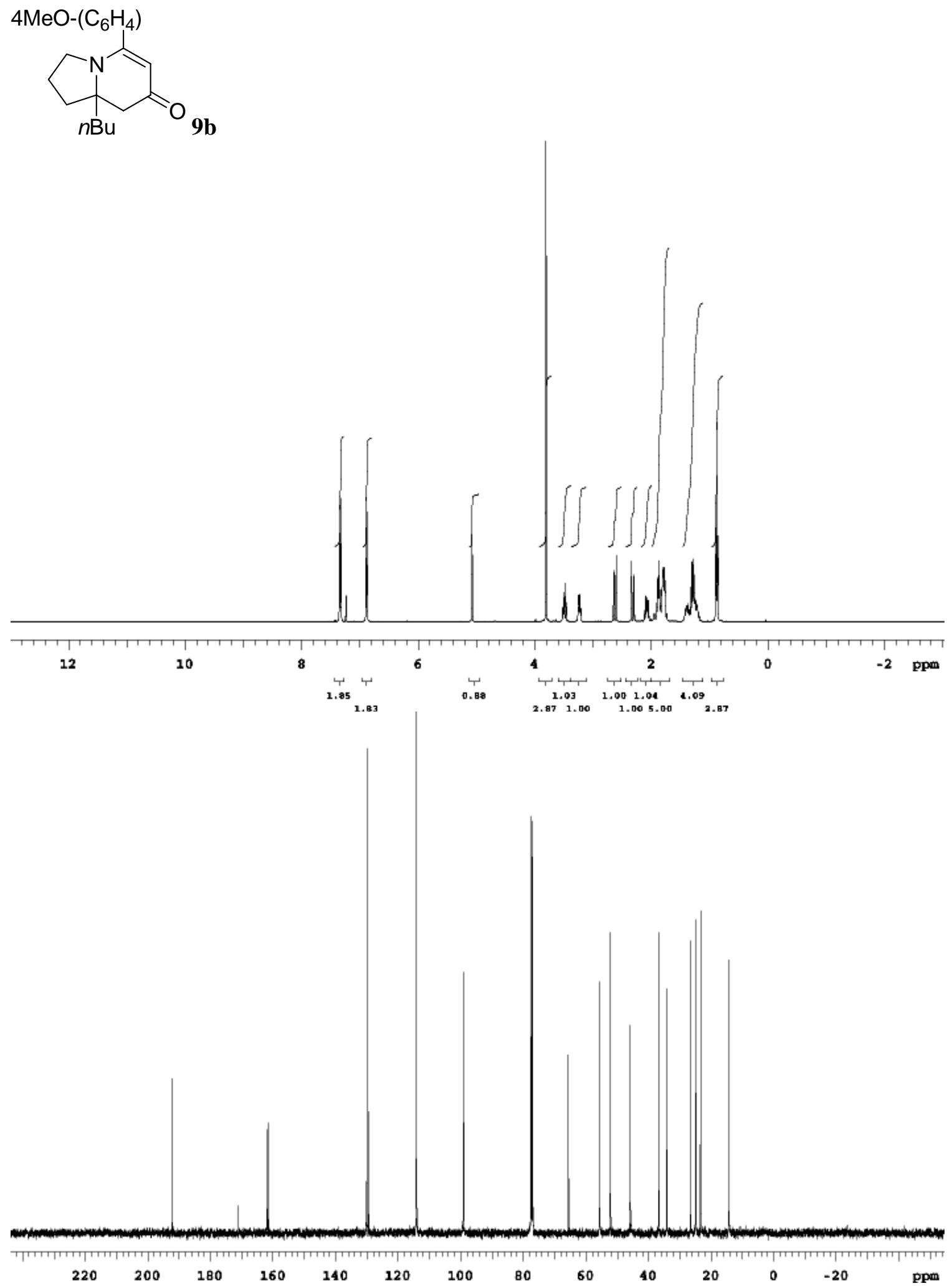

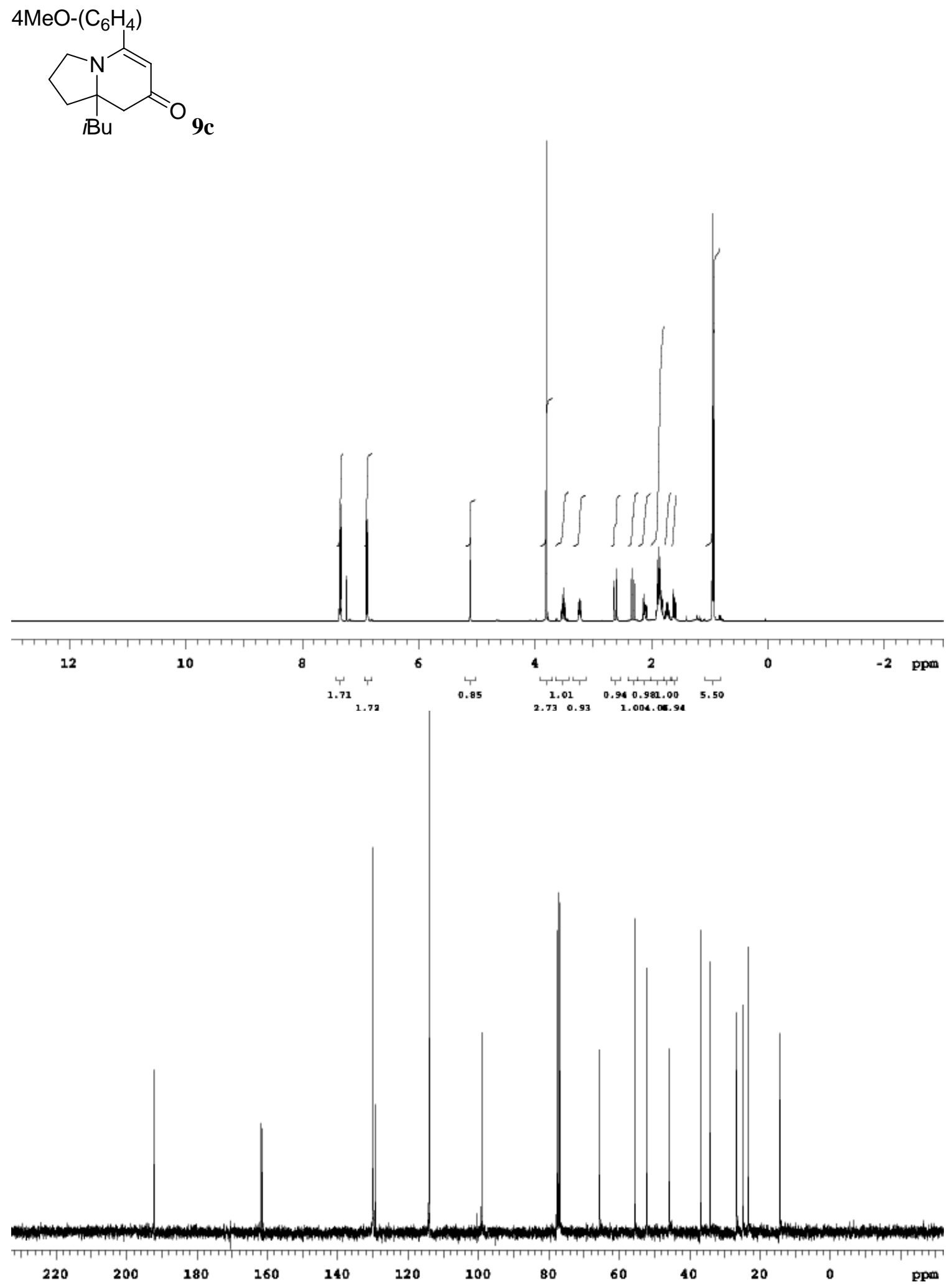

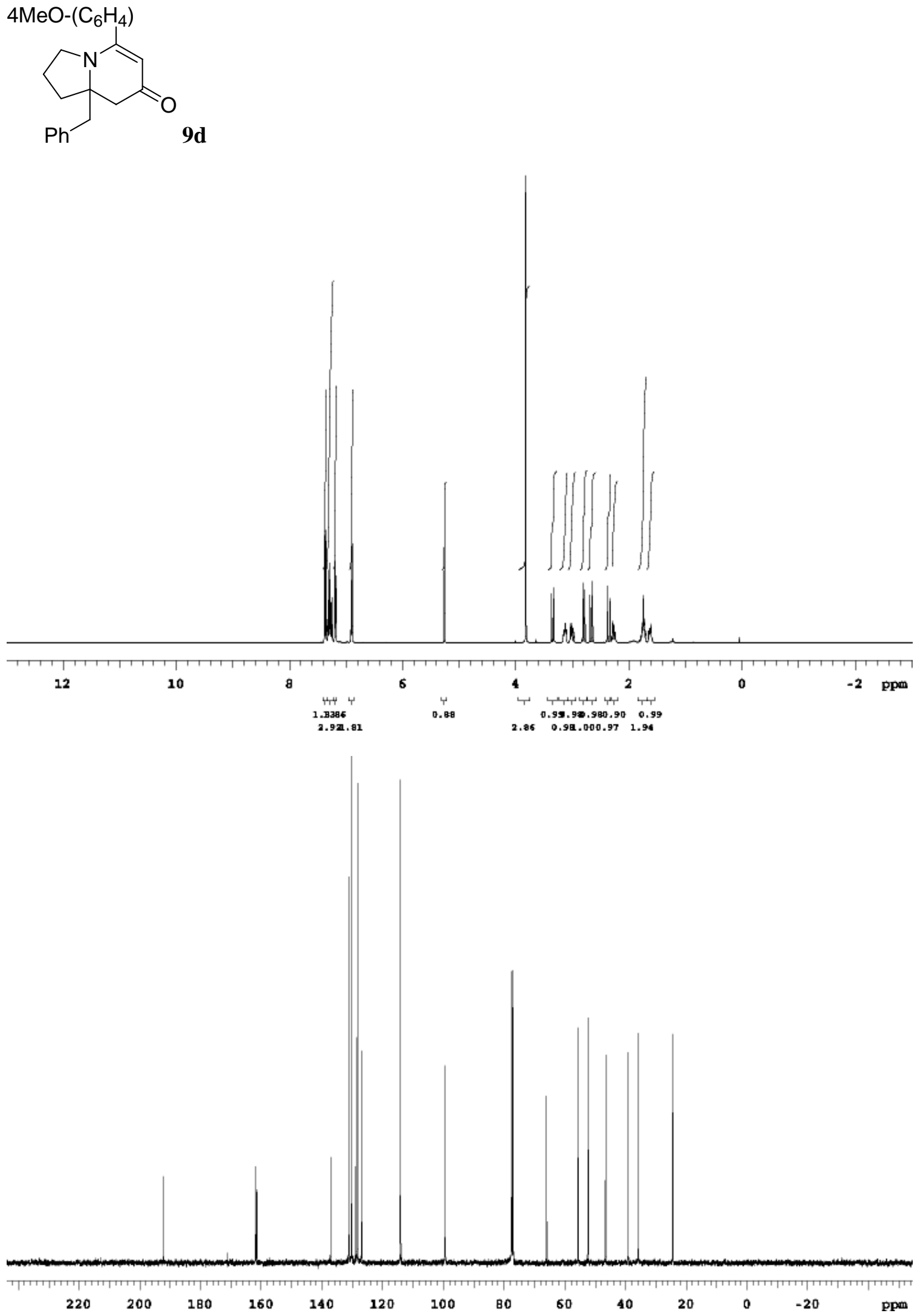

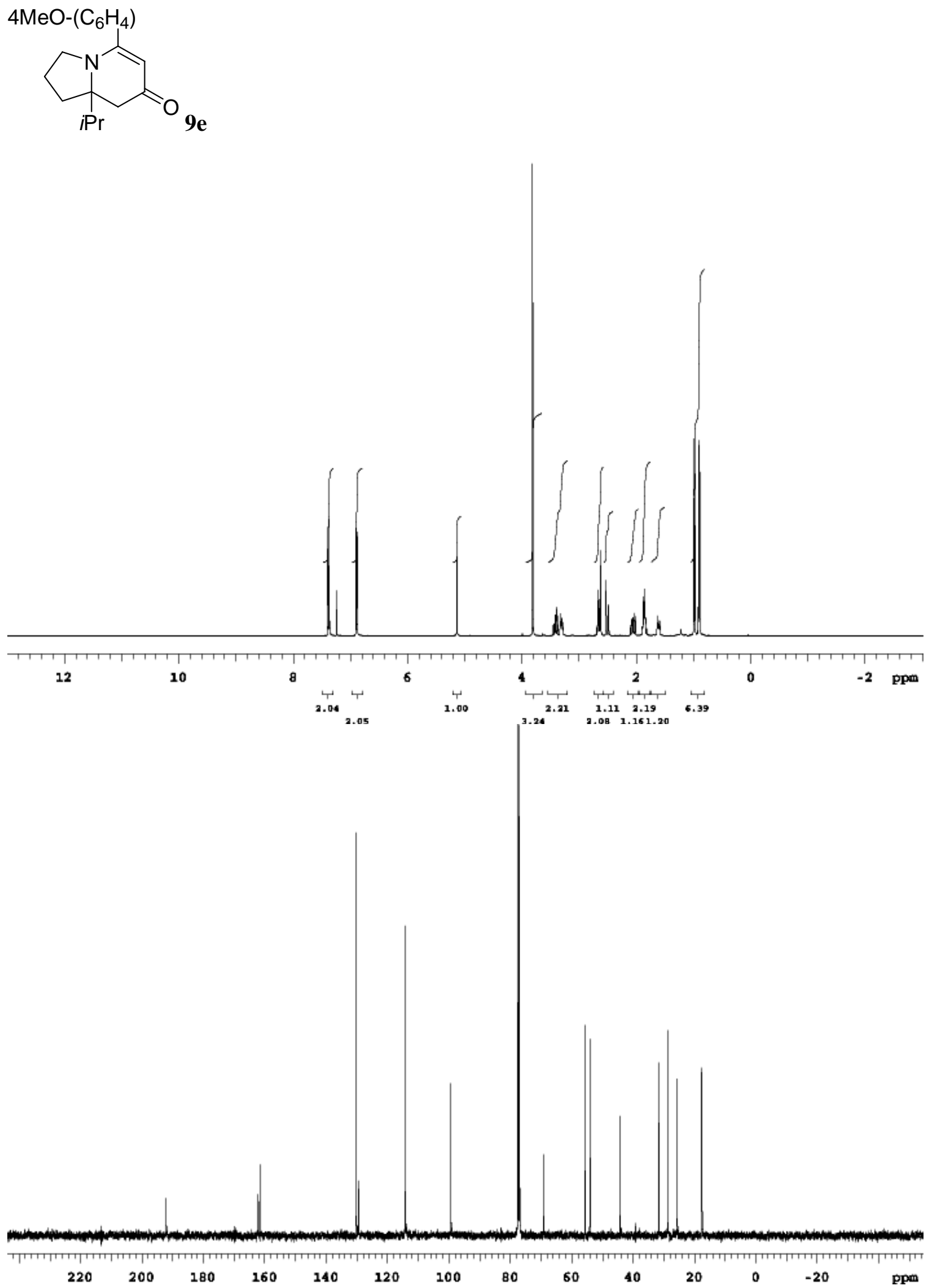

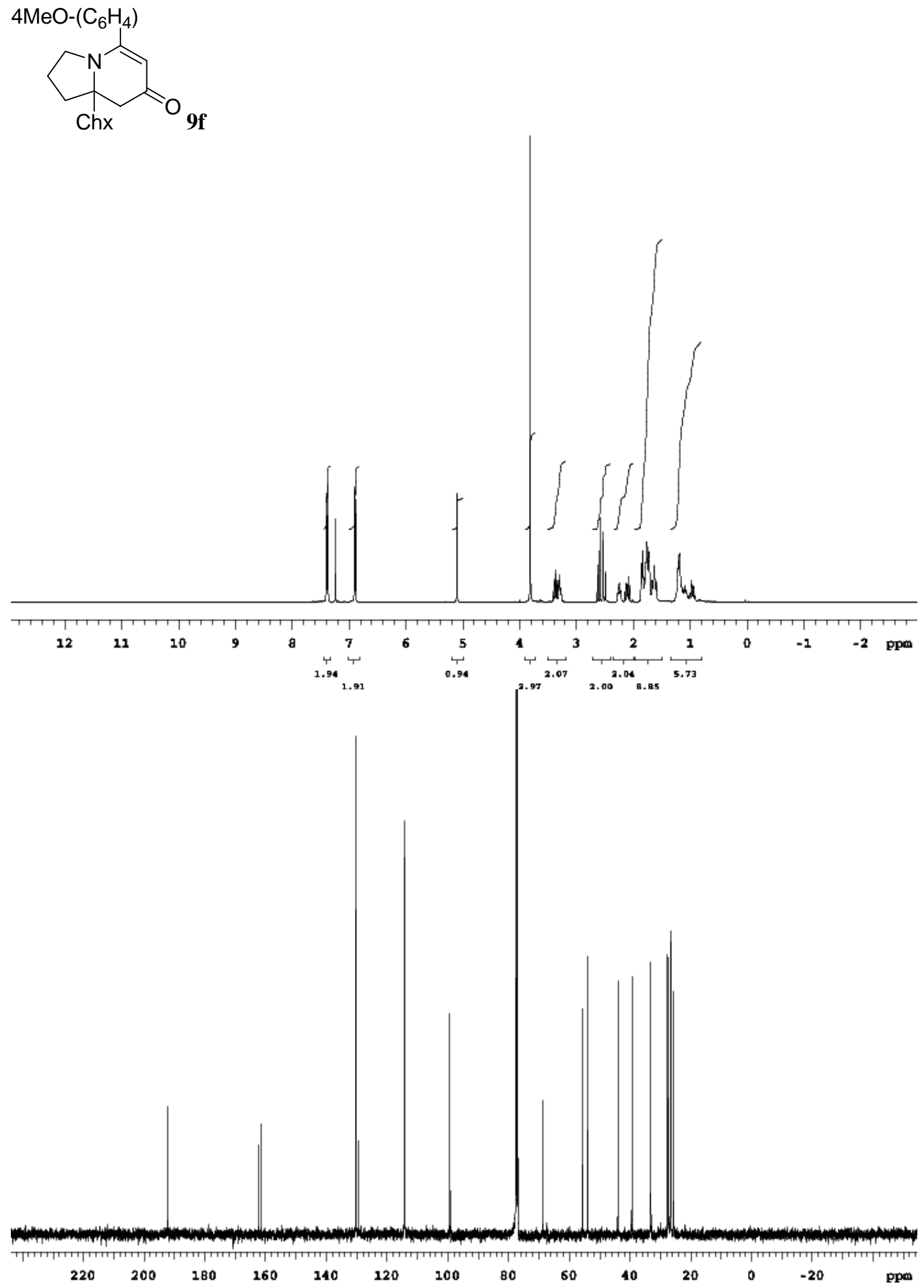

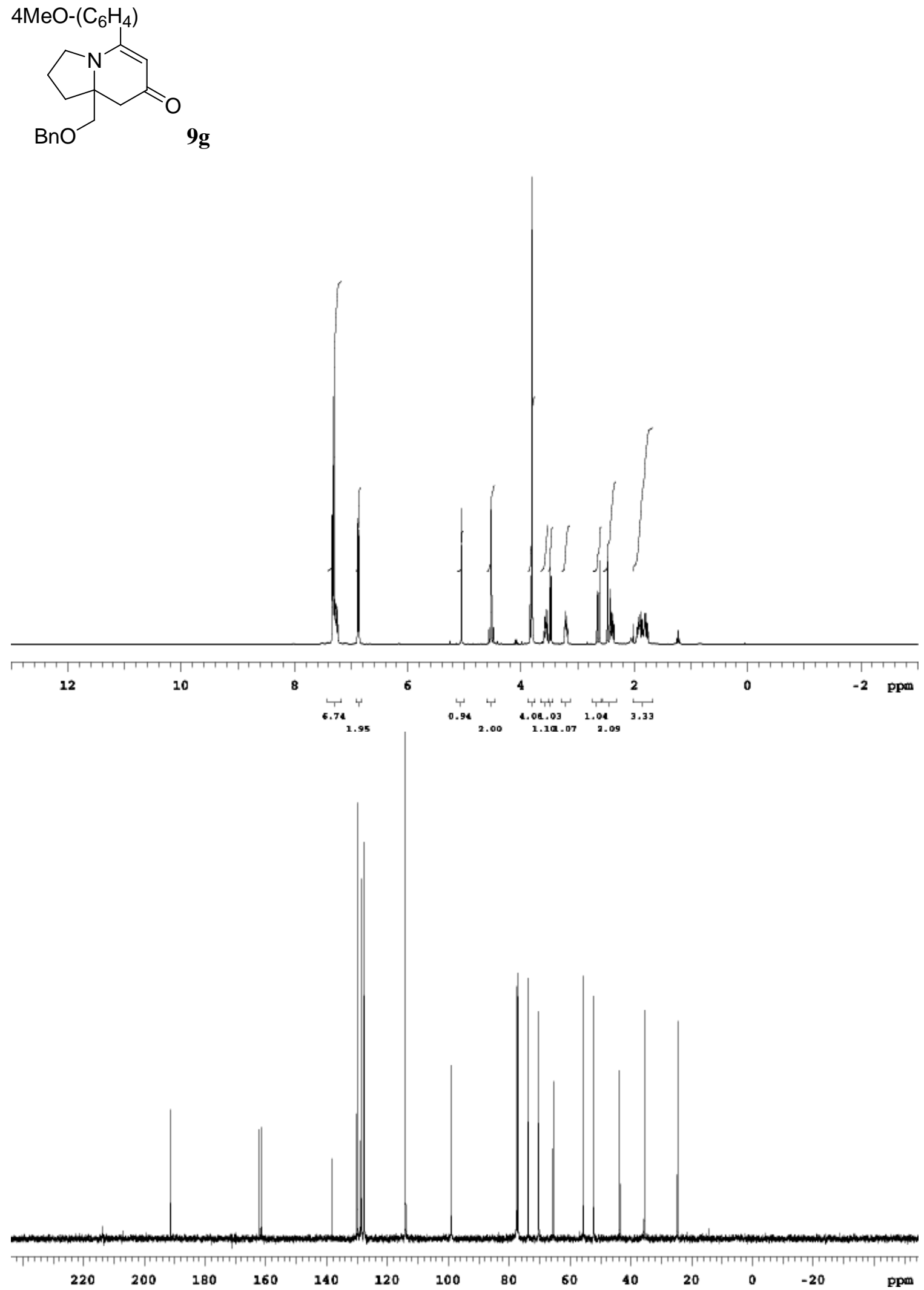

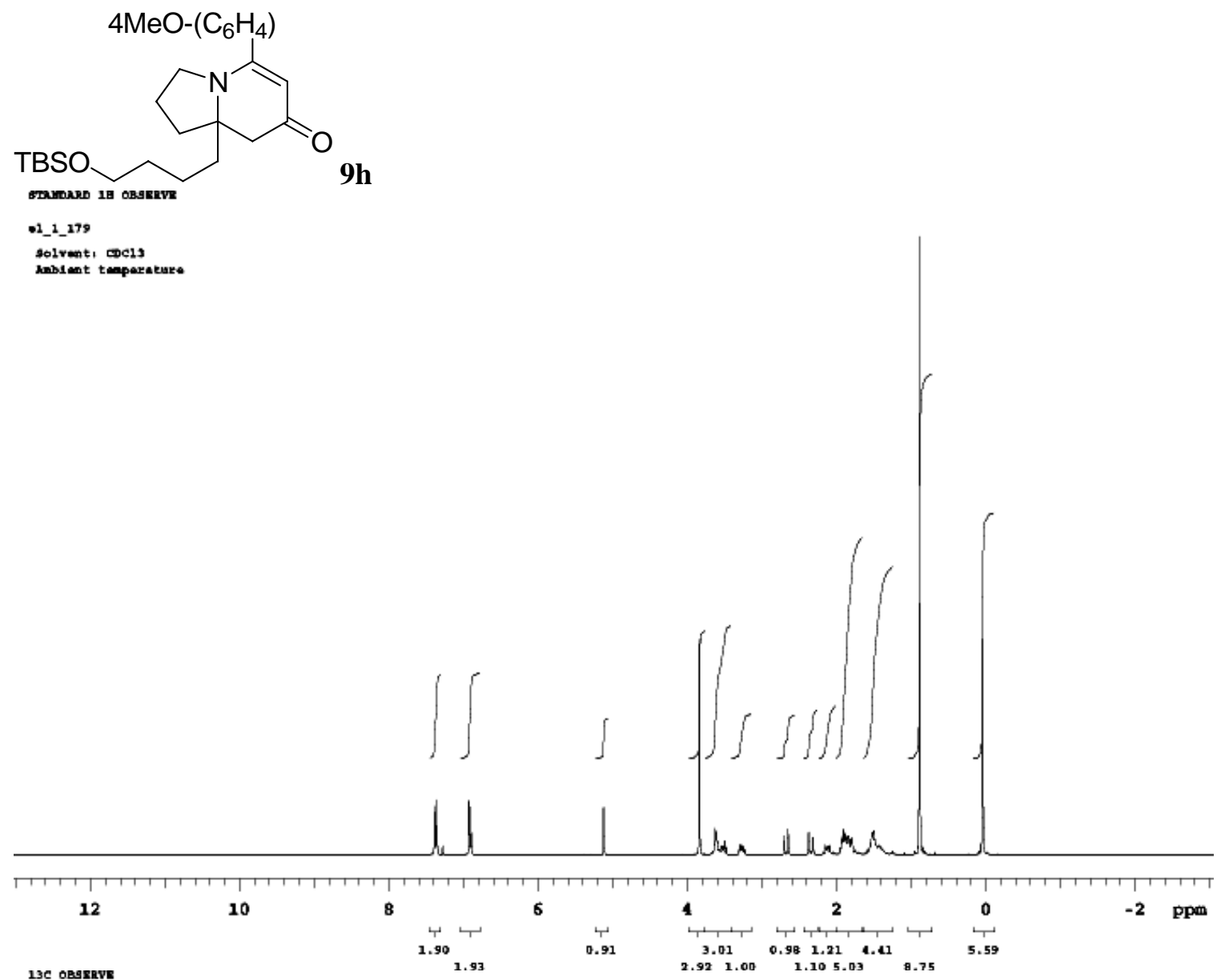

solvent, CDC13

nabient teaporackat.

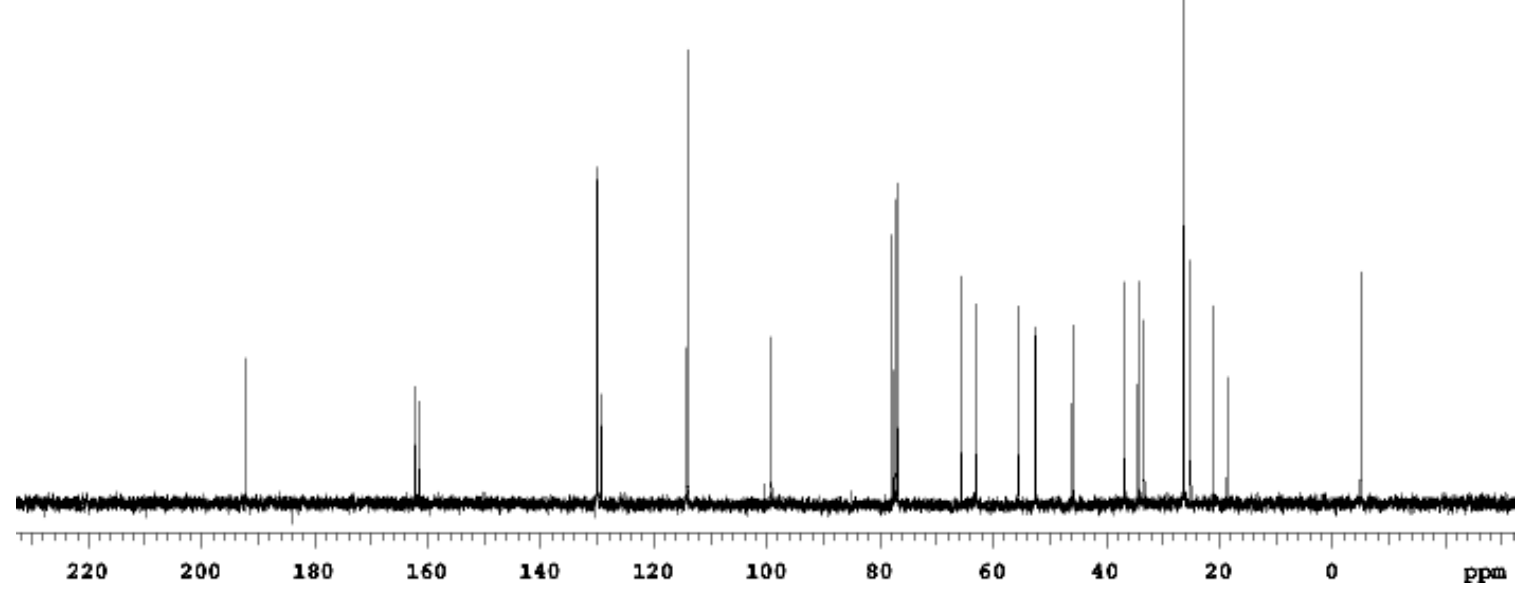




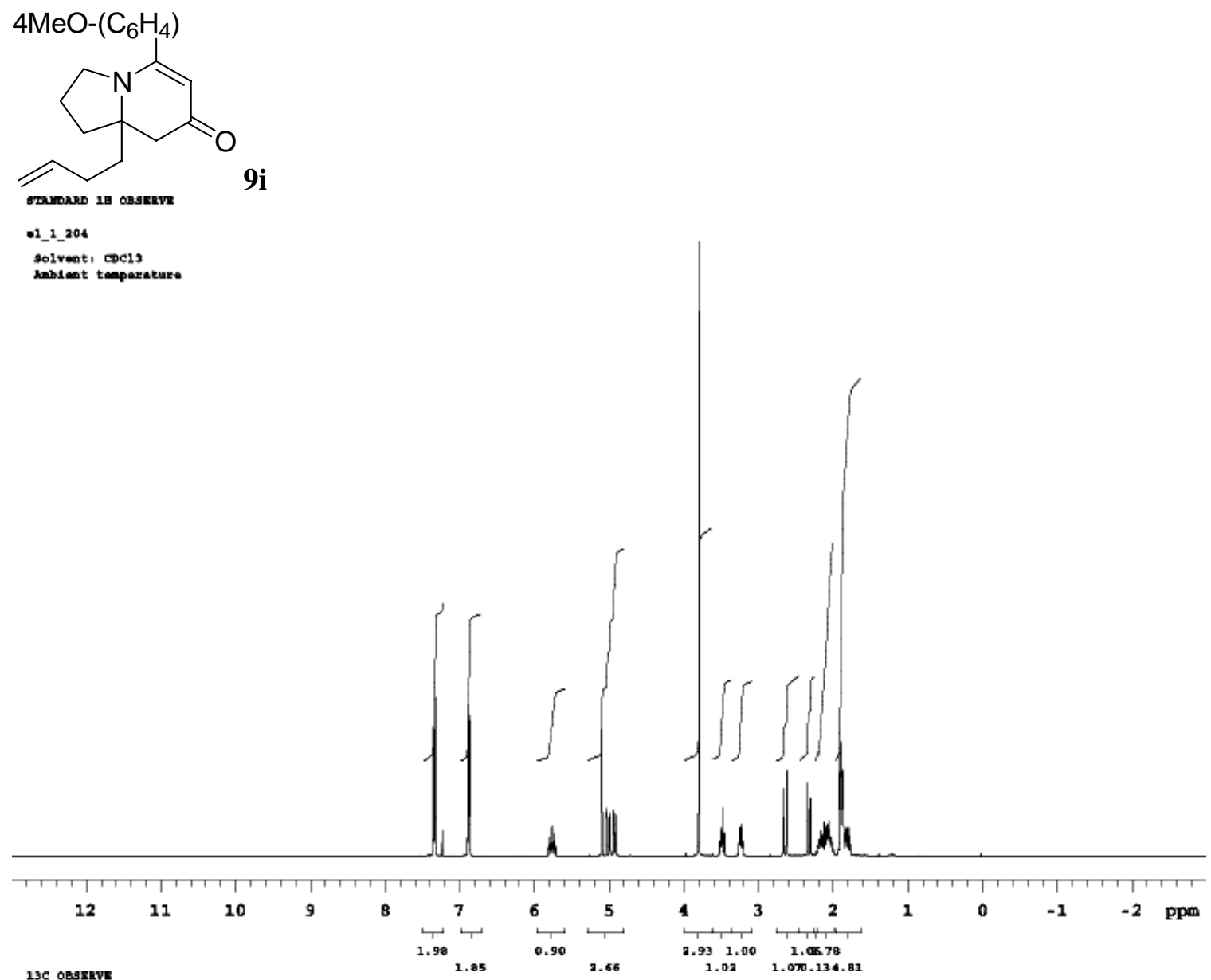

13C OBgrave

-1_2_204

201vent, एDC13

anblent tanparature

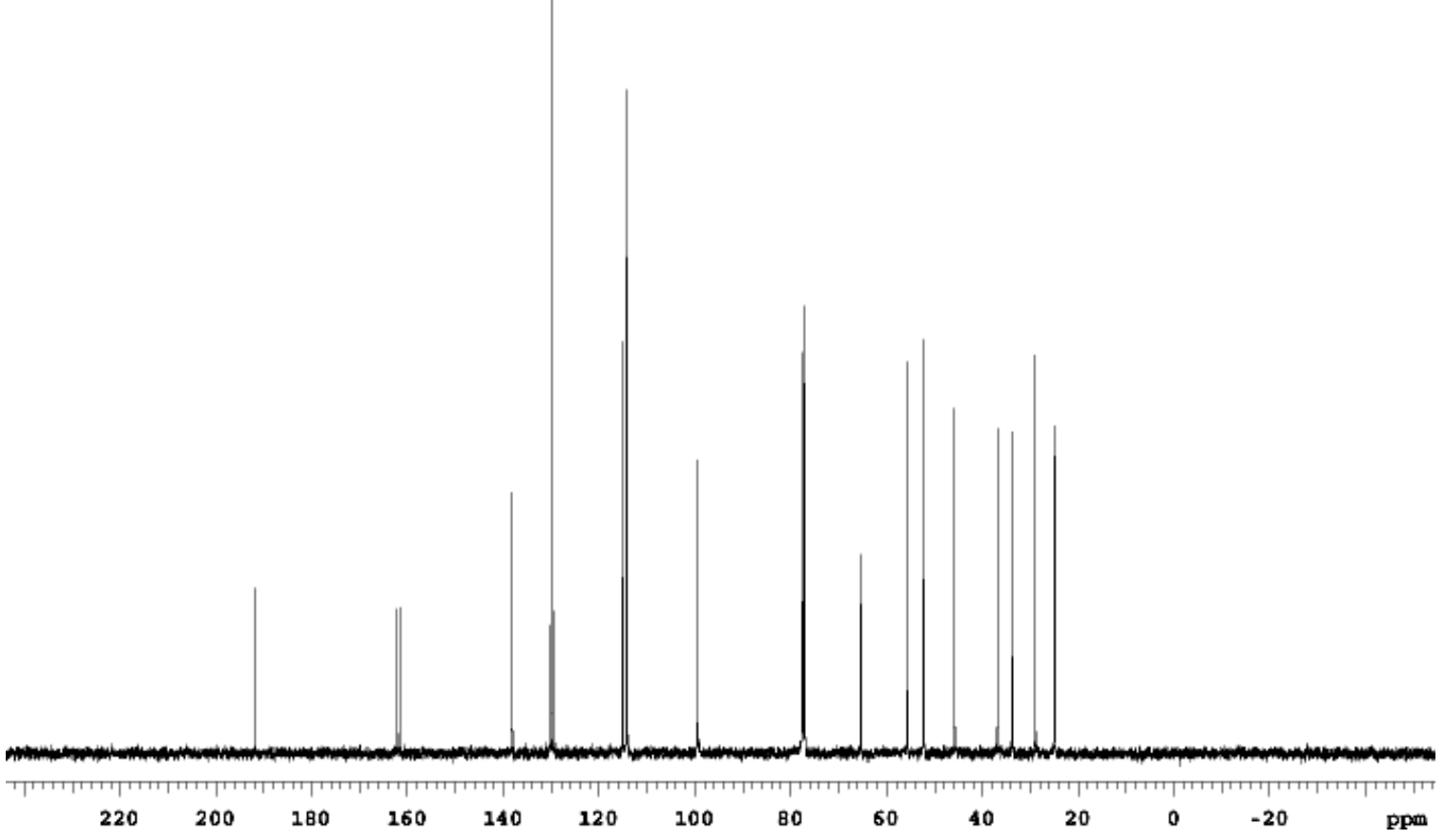




\section{I.10 General procedure for [2+2+2] cycloadditions with Alkyl Alkynes.}

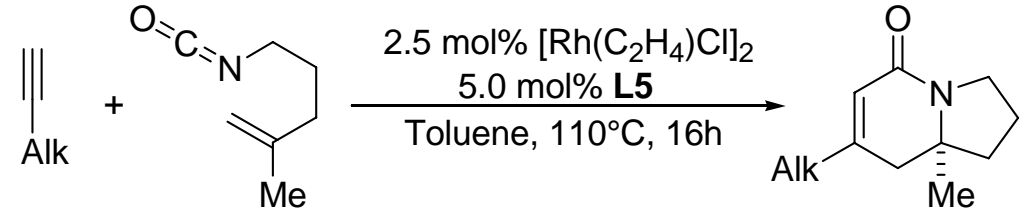

$2(2 \mathrm{eq}) \quad \mathbf{5 a}$

$(R)-6$<smiles></smiles>

(S)-7

In a glovebox under $\mathrm{N}_{2}$ atmosphere, chlorobis(ethylene)rhodium(I) dimer (2.3 $\mathrm{mg}, 0.006 \mathrm{mmol}$ ) and piperidyl-(xylyl-TADDOL)-phosphoramidite L5 (8.3 mg, $0.012 \mathrm{mmol}$ ) were transferred into a round bottom flasked fitted with a reflux condenser. The system was sealed with a standard septum, removed from the glovebox and flushed with Ar. A solution of alkyne $(0.48 \mathrm{mmol})$ and isocyanate $5 \mathbf{a}(0.24 \mathrm{mmol})$ in toluene $(7 \mathrm{~mL})$ was then added. The brown-black solution was then heated to $110^{\circ} \mathrm{C}$ (bath temperature), stirred at reflux for 16 hours under a static atmosphere of Ar, and cooled. The crude mixture was then concentrated and purified by silica gel, column chromatography.

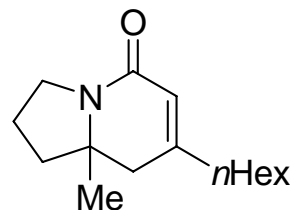

Flash Chromatography (Hexanes:EtOAc;1:3 to 1:8) yielded a clear syrup 6e (76\%) followed by a clear syrup $\mathbf{7 h}(12 \%)$ :

(R)-7-Hexyl-8a-methyl-2,3,8,8a-tetrahydro-1H-indolizin-5-one (6e): $91 \%$ ee by HPLC (Chiralcel ODH, Hex:iPrOH;85:15, $1 \mathrm{ml} / \mathrm{min}, \mathrm{RT}_{\text {major }}=5.2 \mathrm{~min}, \mathrm{RT}_{\text {minor }}=5.7 \mathrm{~min}$ ); $[\alpha]_{\mathrm{D}}=$ $+96.2^{\circ}\left(\mathrm{CHCl}_{3}, \mathrm{c}=1.3\right) ; \mathrm{R}_{f}=0.35$ (100\% EtOAc); IR (Thin Film) v 2928, 2858, 1664, 1615, 1426, 1374, $1338 \mathrm{~cm}^{-1}$; ${ }^{1} \mathrm{H}$ NMR (400 MHz, $\left.\mathrm{CDCl}_{3}\right) \delta 5.67(1 \mathrm{H}, \mathrm{m}), 3.53-3.48(2 \mathrm{H}, \mathrm{m}), 2.33(1 \mathrm{H}, \mathrm{d}, J=$ $16.5 \mathrm{~Hz}), 2.18(1 \mathrm{H}, \mathrm{d}, J=16.5 \mathrm{~Hz}), 2.15-2.08(2 \mathrm{H}, \mathrm{m}), 2.00-1.86(3 \mathrm{H}, \mathrm{m}), 1.80-1.72(1 \mathrm{H}, \mathrm{m})$, 1.46-1.38 (2H, m), 1.30-1.20 (6H, m), $1.12(3 \mathrm{H}, \mathrm{s}), 0.84$ (3H, t, $J=7.0 \mathrm{~Hz}) ;{ }^{13} \mathrm{C}$ NMR $(100 \mathrm{MHz}$, $\left.\mathrm{CDCl}_{3}\right) \delta 163.6,152.3,119.4,60.8,43.7,41.7,40.9,36.9,31.8,29.0,26.7,23.5,22.7,21.6,14.3$; MS (EI) m/e (rel intensity) 236 (100), 234 (14), 220 (11), 178 (3), 154 (4), 149 (4), 136 (5); HRMS (ESI) m/e calcd (M+) 236.2014, found 236.2004.

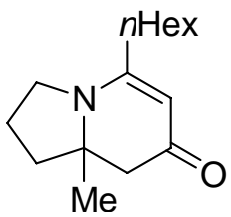

(S)-5-Hexyl-8a-methyl-2,3,8,8a-tetrahydro-1H-indolizin-7-one (7e): Flash Chromatography (Hexanes:EtOAc;1:8) yielded a clear syrup (12\%); 56\% ee by HPLC (Chiralcel ODH, Hex:iPrOH;85:15, $\left.1 \mathrm{ml} / \mathrm{min}, \mathrm{RT}_{\text {major }}=7.5 \mathrm{~min}, \mathrm{RT}_{\text {minor }}=8.4 \mathrm{~min}\right) ;[\alpha]_{\mathrm{D}}=+99.0^{\circ}\left(\mathrm{CHCl}_{3}, \mathrm{c}=0.8\right)$; $\mathrm{R}_{f}=0.15$ (100\% EtOAc); IR (Thin Film) v 2957, 2928, 2870, 1625, 1544, 1487, 1267, 1213, 
$1100 \mathrm{~cm}^{-1} ;{ }^{1} \mathrm{H}$ NMR (400 MHz, $\left.\mathrm{CDCl}_{3}\right) \delta 4.89(1 \mathrm{H}, \mathrm{s}), 3.60-3.52(1 \mathrm{H}, \mathrm{m}), 3.47-3.39(1 \mathrm{H}, \mathrm{m})$, $2.48(1 \mathrm{H}, \mathrm{d}, J=16.0 \mathrm{~Hz}), 2.28(1 \mathrm{H}, \mathrm{d}, J=16.0 \mathrm{~Hz}), 2.20-2.10(2 \mathrm{H}, \mathrm{m}), 2.08-1.94(3 \mathrm{H}, \mathrm{m}), 1.84-$ $1.74(1 \mathrm{H}, \mathrm{m}), 1.52-1.40(2 \mathrm{H}, \mathrm{m}), 1.35-1.20(6 \mathrm{H}, \mathrm{m}), 1.17(3 \mathrm{H}, \mathrm{s}), 0.85(3 \mathrm{H}, \mathrm{t}, J=7.0 \mathrm{~Hz}) ;{ }^{13} \mathrm{C}$ NMR (100 MHz, $\left.\mathrm{CDCl}_{3}\right) \delta 191.1,163.2,96.2,63.1,48.0,46.5,39.7,34.1,31.7,29.1,27.3,22.7$, 22.4, 20.0, 14.2; MS (EI) m/e (rel intensity) 236 (100), 234 (15), 220 (12), 165 (14), 150 (8), 132 (16); HRMS (ESI) m/e calcd $\left(\mathrm{M}^{+}\right)$236.2014, found 236.2011.

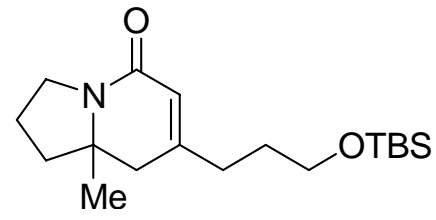

Flash Chromatography (Hex:EtOAc;1:2 to EtOAc 100\%) yielded a brown syrup 6f (63\%) followed by a light yellow syrup 7i (7\%):

(R)-7-[3-(tert-Butyl-dimethyl-silanyloxy)-propyl]-8a-methyl-2,3,8,8a-tetrahydro-1Hindolizin-5-one (6f): 95\% ee by HPLC (Chiralcel ODH, Hex:iPrOH;85:15, $1 \mathrm{ml} / \mathrm{min}, \mathrm{RT}_{\text {major }}=$ $\left.8.7 \mathrm{~min}, \mathrm{RT}_{\text {minor }}=10.1 \mathrm{~min}\right) ;:[\alpha]_{\mathrm{D}}=+90.0^{\circ}\left(\mathrm{CHCl}_{3}, \mathrm{c}=0.8\right) ; \mathrm{R}_{f}=0.45$ (EtOAc $100 \%$ ); IR (Thin Film) v 2954, 2928, 2857, 1664, 1616, 1461, 1427, 1103, $835 \mathrm{~cm}^{-1} ;{ }^{1} \mathrm{H}$ NMR (400 MHz, $\left.\mathrm{CDCl}_{3}\right)$ $\delta 5.69(1 \mathrm{H}, \mathrm{t}, J=1.0 \mathrm{~Hz}), 3.59(2 \mathrm{H}, \mathrm{t}, J=6.0 \mathrm{~Hz}), 3.55-3.46(2 \mathrm{H}, \mathrm{m}), 2.36(1 \mathrm{H}, \mathrm{d}, J=17.0 \mathrm{~Hz})$, 2.20 (1H, d, $J=17.0 \mathrm{~Hz}$ ), 2.20-2.15 (2H, m), 1.99-1.89 (3H, m), 1.75-1.63 (3H, m), 1.13 (3H, s), 0.85 (9H, s), $0.00(6 \mathrm{H}, \mathrm{s}) ;{ }^{13} \mathrm{C}$ NMR $\left(100 \mathrm{MHz} \mathrm{CDCl}_{3}\right) \delta 163.5,151.9,119.4,62.5,60.8,43.7$, 41.9, 40.9, 33.3, 30.0, 26.1, 23.6, 21.6, 18.5, -5.1; HRMS (ESI) $\mathrm{m} / e$ calcd $\left(\mathrm{M}+\mathrm{H}^{+}\right)$324.2353, found 324.2350 .

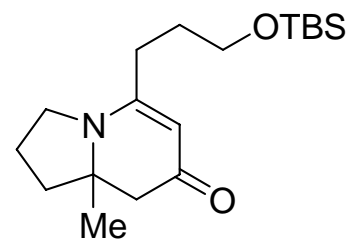

(S)-5-[3-(tert-Butyl-dimethyl-silanyloxy)-propyl]-8a-methyl-2,3,8,8a-tetrahydro-1H-

indolizin-7-one (7f): $64 \%$ ee by HPLC (Chiralcel ODH, Hex:iPrOH;90:10, $1 \mathrm{ml} / \mathrm{min}, \mathrm{RT}_{\text {major }}=$ $\left.7.9 \mathrm{~min}, \mathrm{RT}_{\text {minor }}=9.0 \mathrm{~min}\right) ;[\alpha]_{\mathrm{D}}=+74.0^{\circ}\left(\mathrm{CHCl}_{3}, \mathrm{c}=0.4\right) ; \mathrm{R}_{f}=0.15($ EtOAc $100 \%) ;$ IR $($ Thin Film) v 2955, 2928, 1626, 1545, 1472, $1099 \mathrm{~cm}^{-1} ;{ }^{1} \mathrm{H}$ NMR (400 MHz, $\left.\mathrm{CDCl}_{3}\right) \delta 4.88(1 \mathrm{H}, \mathrm{s})$, $3.62(2 \mathrm{H}, \mathrm{t}, J=6.0 \mathrm{~Hz}), 3.62-2.56(1 \mathrm{H}, \mathrm{m}), 3.49-3.41(1 \mathrm{H}, \mathrm{ddd}, J=10.0,8.0,8.0 \mathrm{~Hz}), 2.48(1 \mathrm{H}$, d, $J=16.0 \mathrm{~Hz}), 2.28(1 \mathrm{H}, \mathrm{d}, J=16.0 \mathrm{~Hz}), 2.26(2 \mathrm{H}, \mathrm{ddd}, J=12.0,7.5,3.0 \mathrm{~Hz}), 2.07-1.95(3 \mathrm{H}$, m), 1.84-1.75 (1H, m), 1.71-1.65 (2H, m), $1.18(3 \mathrm{H}, \mathrm{s}), 0.86(9 \mathrm{H}, \mathrm{s}), 0.02(6 \mathrm{H}, \mathrm{s}) ;{ }^{13} \mathrm{C}$ NMR (100 $\left.\mathrm{MHz}, \mathrm{CDCl}_{3}\right) \delta 191.2$, 162.9, 96.0, 63.1, 62.0, 48.0, 46.5, 39.7, 30.5, 30.4, 29.9, 26.1, 22.4, 20.0, -5.2; HRMS (ESI) $\mathrm{m} / \mathrm{e}$ calcd $\left(\mathrm{M}^{+}\right)$324.2353, found 324.2355.

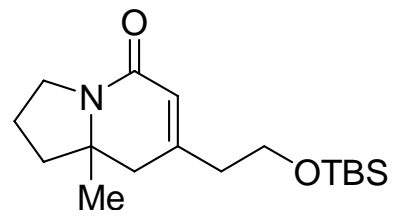

(R)-7-[2-(tert-Butyl-dimethyl-silanyloxy)-ethyl]-8a-methyl-2,3,8,8a-tetrahydro-1H-indolizin5-one (6g): Flash Chromatography (Hexanes:EtOAc;1:5) yielded a light yellow syrup (77\%); 93\% ee by HPLC (Chiralcel ADH, Hex:iPrOH;90:10, $1 \mathrm{ml} / \mathrm{min}, \mathrm{RT}_{\text {major }}=5.9 \mathrm{~min}, \mathrm{RT}_{\text {minor }}=5.2$ $\min ) ;[\alpha]_{\mathrm{D}}=+104.0^{\circ}\left(\mathrm{CHCl}_{3}, \mathrm{c}=1.1\right) ; \mathrm{R}_{f}=0.48$ (EtOAc 100\%); IR (Thin Film) $v 2955,2928$, 
2857, 1664, 1616, 1427, 1255, 1096, $837 \mathrm{~cm}^{-1} ;{ }^{1} \mathrm{H}$ NMR (400 MHz, $\left.\mathrm{CDCl}_{3}\right) \delta 5.71(1 \mathrm{H}, \mathrm{d}, J=$ $1.0 \mathrm{~Hz}), 3.73(2 \mathrm{H}, \mathrm{t}, J=6.5 \mathrm{~Hz}), 3.55-3.50(2 \mathrm{H}, \mathrm{m}), 2.40-2.27(4 \mathrm{H}, \mathrm{m}), 2.00-1.89(3 \mathrm{H}, \mathrm{m}), 1.78-$ $1.70(1 \mathrm{H}, \mathrm{m}), 1.14(3 \mathrm{H}, \mathrm{s}), 0.84(9 \mathrm{H}, \mathrm{s}), 0.02(3 \mathrm{H}, \mathrm{s}), 0.01(3 \mathrm{H}, \mathrm{s}) ;{ }^{13} \mathrm{C}$ NMR $\left(100 \mathrm{MHz}, \mathrm{CDCl}_{3}\right)$ $\delta$ 163.4, 149.6, 121.0, 61.2, 60.9, 43.7, 42.0, 40.9, 40.6, 26.1, 23.6, 21.6, 18.4, -5.2; HRMS (ESI) $\mathrm{m} / \mathrm{e}$ calcd $\left(\mathrm{M}^{+}\right)$310.2197, found 310.2187.

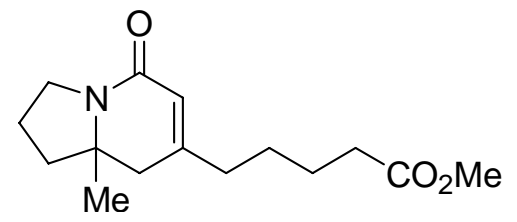

Flash Chromatography (Hexanes:EtOAc;1:5 then EtOAc 100\%) yielded a light yellow syrup 6k (74\%) followed by a light yellow syrup $7 \mathbf{h}(9 \%)$ :

(R)-5-(8a-Methyl-5-oxo-1,2,3,5,8,8a-hexahydro-indolizin-7-yl)-pentanoic acid methyl ester (6h): 93\% ee by HPLC (Chiralcel ODH, Hex:iPrOH;90:10, $1 \mathrm{ml} / \mathrm{min}, \mathrm{RT}_{\text {major }}=16.4 \mathrm{~min}, \mathrm{RT}_{\text {minor }}$ $=18.0 \mathrm{~min}) ;[\alpha]_{\mathrm{D}}=+111.0^{\circ}\left(\mathrm{CHCl}_{3}, \mathrm{c}=0.9\right) ; \mathrm{R}_{f}=0.48($ EtOAc 100\%); IR (Thin Film) $v 2949$, 2877, 1736, 1660, 1612, $1432 \mathrm{~cm}^{-1} ;{ }^{1} \mathrm{H}$ NMR (400 MHz, $\left.\mathrm{CDCl}_{3}\right) \delta 5.68(1 \mathrm{H}, \mathrm{s}), 3.62(3 \mathrm{H}, \mathrm{s})$, 3.53-3.47 (2H, m), $2.34(1 \mathrm{H}, \mathrm{d}, J=17.0 \mathrm{~Hz}), 2.29(2 \mathrm{H}$, ddd, $J=7.0,7.0,4.0 \mathrm{~Hz}), 2.18(1 \mathrm{H}, \mathrm{d}, J=$ $17.0 \mathrm{~Hz}$ ), $2.13(2 \mathrm{H}, \mathrm{dd}, J=7.5,7.5 \mathrm{~Hz}), 1.99-1.87$ (3H, m), 1.78-1.73 (1H, m), 1.65-1.57 (2H, m), 1.52-1.44 (2H, m), 1.12 (3H, s); ${ }^{13} \mathrm{C}$ NMR (100 MHz, $\left.\mathrm{CDCl}_{3}\right) \delta 174.0,163.4,151.4,119.7$, 60.8, 51.7, 43.7, 41.7, 40.9, 36.5, 33.9, 26.2, 24.6, 23.6, 21.5; HRMS (ESI) m/e calcd $\left(\mathrm{M}^{+}\right)$ 266.1751, found 266.1764 .

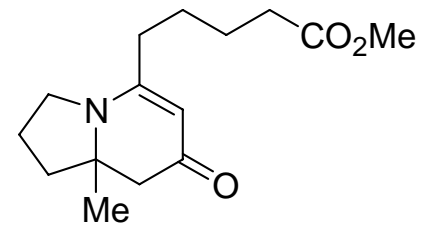

(S)-5-(8a-Methyl-7-oxo-1,2,3,7,8,8a-hexahydro-indolizin-5-yl)-pentanoic acid methyl ester (7h): 49\% ee by HPLC (Chiralcel ADH, Hex: $\mathrm{PrOH} ; 80: 20,1 \mathrm{ml} / \mathrm{min}, \mathrm{RT}_{\text {major }}=8.6 \mathrm{~min}, \mathrm{RT}_{\text {minor }}=$ $8.1 \mathrm{~min}) ;[\alpha]_{\mathrm{D}}=49.0^{\circ}\left(\mathrm{CHCl}_{3}, \mathrm{c}=0.5\right) ; \mathrm{R}_{f}=0.19$ (EtOAc 100\%); IR (Thin Film) v 2953, 2871, 1736, 1621, 1555, 1484, 1269, 1212, $1168 \mathrm{~cm}^{-1} ;{ }^{1} \mathrm{H}$ NMR (400 MHz, $\left.\mathrm{CDCl}_{3}\right) \delta 4.87$ (1H, s), 3.64 (3H, s), 3.55 (1H, ddd, $J=11.0,8.0,6.0 \mathrm{~Hz}), 3.42$ (1H, ddd, $J=11.0,8.0,8.0 \mathrm{~Hz}), 2.46(1 \mathrm{H}, \mathrm{d}, J$ $=16.0 \mathrm{~Hz}), 2.31(2 \mathrm{H}, \mathrm{t}, J=7.0 \mathrm{~Hz}), 2.28(1 \mathrm{H}, \mathrm{d}, J=16.0 \mathrm{~Hz}), 2.24-2.14(2 \mathrm{H}, \mathrm{m}), 2.06-1.95(3 \mathrm{H}$, $\mathrm{m}), 1.83-1.74(1 \mathrm{H}, \mathrm{m}), 1.70-1.62(2 \mathrm{H}, \mathrm{m}), 1.57-1.49(2 \mathrm{H}, \mathrm{m}), 1.17(3 \mathrm{H}, \mathrm{s}) ;{ }^{13} \mathrm{C}$ NMR $(100 \mathrm{MHz}$, $\left.\mathrm{CDCl}_{3}\right) \delta 191.4,173.9,162.2,96.2,63.2,51.8,48.1,46.5,39.7,33.8,33.7,26.7,24.7,22.5,20.0$; HRMS (ESI) $m / e$ calcd $\left(\mathrm{M}+\mathrm{H}^{+}\right)$266.1751, found 266.1739.

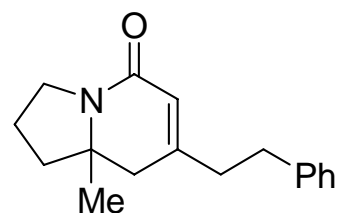

(R)-8a-Methyl-7-phenethyl-2,3,8,8a-tetrahydro-1H-indolizin-5-one $\quad$ (6i): $\quad$ Flash Chromatography (Hexanes:EtOAc;1:4) yielded a light yellow syrup (71\%); 95\% ee by HPLC (Chiralcel ADH, Hex:iPrOH;90:10, $1 \mathrm{ml} / \mathrm{min}, \mathrm{RT}_{\text {major }}=12.9 \mathrm{~min}, \mathrm{RT}_{\text {minor }}=10.4 \mathrm{~min}$ ); $[\alpha]_{\mathrm{D}}=$ $+110.0^{\circ}\left(\mathrm{CHCl}_{3}, \mathrm{c}=0.8\right) ; \mathrm{R}_{f}=0.44$ (EtOAc 100\%); IR (Thin Film) v 2967, 2925, 2881, 1661, 1613, $1429 \mathrm{~cm}^{-1}$; ${ }^{1} \mathrm{H}$ NMR (400 MHz, $\left.\mathrm{CDCl}_{3}\right) \delta$ 7.28-7.23 (2H, m), 7.19-7.15 (3H, m), $5.75(1 \mathrm{H}$, 
bs), 3.55-3.50 (2H, m), 2.78 (2H, ddd, $J=9.0,9.0,4.5 \mathrm{~Hz}$ ), 2.45 (2H, td, $J=9.0,1.0 \mathrm{~Hz}$ ), 2.38 $(1 \mathrm{H}, \mathrm{d}, J=16.0 \mathrm{~Hz}), 2.24(1 \mathrm{H}, \mathrm{d}, J=16.0 \mathrm{~Hz}), 2.00-1.88(3 \mathrm{H}, \mathrm{m}), 1.81-1.70(1 \mathrm{H}, \mathrm{m}), 1.12(3 \mathrm{H}$, s); ${ }^{13} \mathrm{C}$ NMR $\left(100 \mathrm{MHz}, \mathrm{CDCl}_{3}\right) \delta 163.4,151.3,141.0,128.7,128.4,126.4,119.8,60.9,43.7$, 41.9, 40.9, 38.4, 33.2, 23.6, 21.5; HRMS (ESI) $\mathrm{m} / \mathrm{e}$ calcd $\left(\mathrm{M}+\mathrm{H}^{+}\right)$256.1696, found 256.1696.

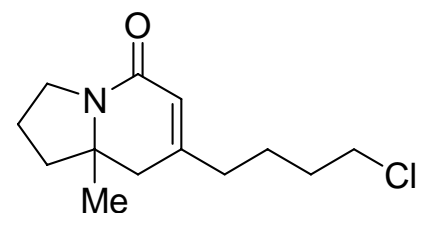

(R)-7-(4-Chloro-butyl)-8a-methyl-2,3,8,8a-tetrahydro-1H-indolizin-5-one $\quad(\mathbf{6 j}): \quad$ Flash Chromatography (Hex:EtOAc;1:2) yielded a yellowish syrup (60\%); 92\% ee by HPLC (Chiralcel $\mathrm{ODH}$, Hex:iPrOH;85:15, $\left.1 \mathrm{ml} / \mathrm{min}, \mathrm{RT}_{\text {major }}=8.7 \mathrm{~min}, \mathrm{RT}_{\text {minor }}=10.1 \mathrm{~min}\right) ;[\alpha]_{\mathrm{D}}=+106.0^{\circ}$ $\left(\mathrm{CHCl}_{3}, \mathrm{c}=0.53\right) ; \mathrm{R}_{f}=0.52$ (EtOAc 100\%); IR (Thin Film) $v 2967,1660,1605,1433 \mathrm{~cm}^{-1} ;{ }^{1} \mathrm{H}$ NMR (400 MHz, CDCl $)_{3} \delta 5.71(1 \mathrm{H}, \mathrm{t}, J=1.0 \mathrm{~Hz}), 3.58-3.47(4 \mathrm{H}, \mathrm{m}), 2.36(1 \mathrm{H}, \mathrm{d}, J=17.0 \mathrm{~Hz})$, $2.21(1 \mathrm{H}, \mathrm{d}, J=17.0 \mathrm{~Hz}), 2.17(2 \mathrm{H}, \mathrm{t}, J=7.5 \mathrm{~Hz}), 2.01-1.89(2 \mathrm{H}, \mathrm{m}), 1.82-1.73(4 \mathrm{H}, \mathrm{m}), 1.66-$ 1.57 (2H, m), $1.14(3 \mathrm{H}, \mathrm{s}) ;{ }^{13} \mathrm{C}$ NMR $\left(100 \mathrm{MHz}, \mathrm{CDCl}_{3}\right) \delta 163.4,151.2,119.9,60.8,44.8,43.7$, 41.6, 40.9, 36.0, 32.0, 23.9, 23.6, 21.6; HRMS (ESI) m/e calcd $\left(\mathrm{M}+\mathrm{H}^{+}\right)$242.1306, found 242.1308. 


\section{I.11 ${ }^{1} \mathrm{H}$ and ${ }^{13} \mathrm{C}$ NMR Spectra for [2+2+2] Products from Alkyl Alkynes}
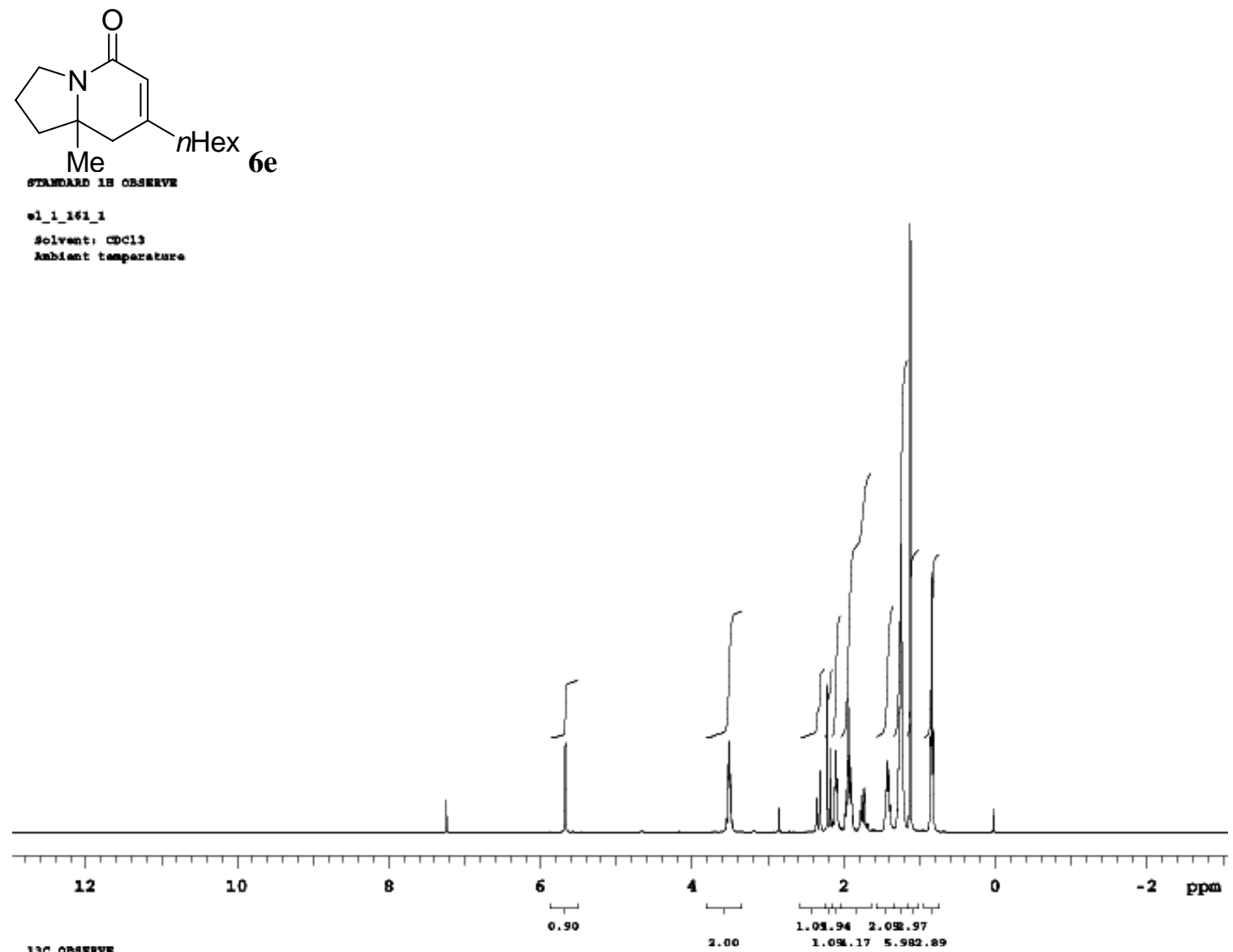

13e obsreve

-1_1_161_1

solvent, CDC1s

knblent temparakar.

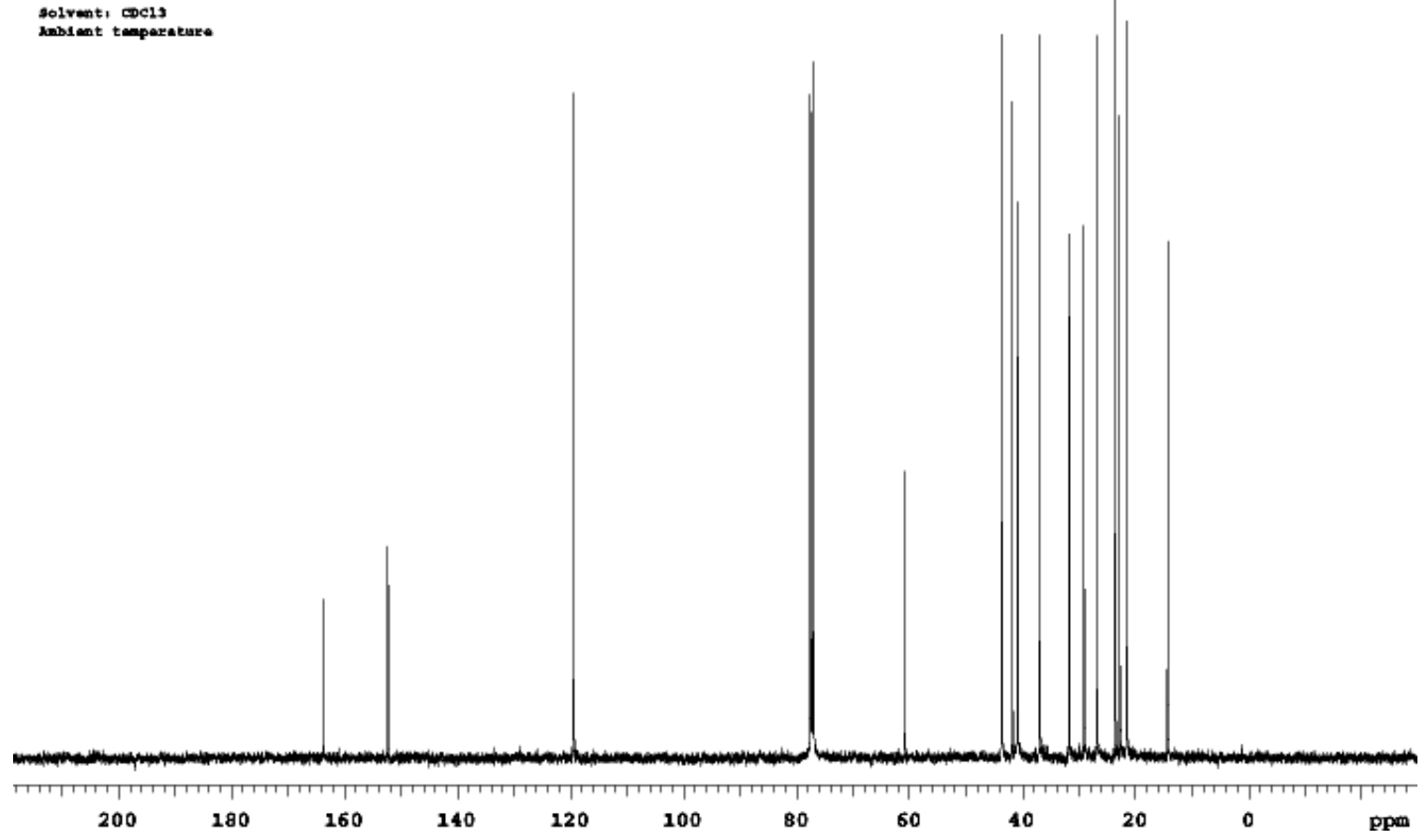




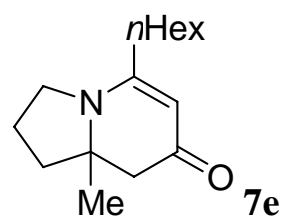

GRumane 18 OBgenve

Solvent, CDC1s

nablent tmaperaters. 
<smiles>CC12CCCN1C(=O)C=C(CCC[OH+][SnH3])C2</smiles>
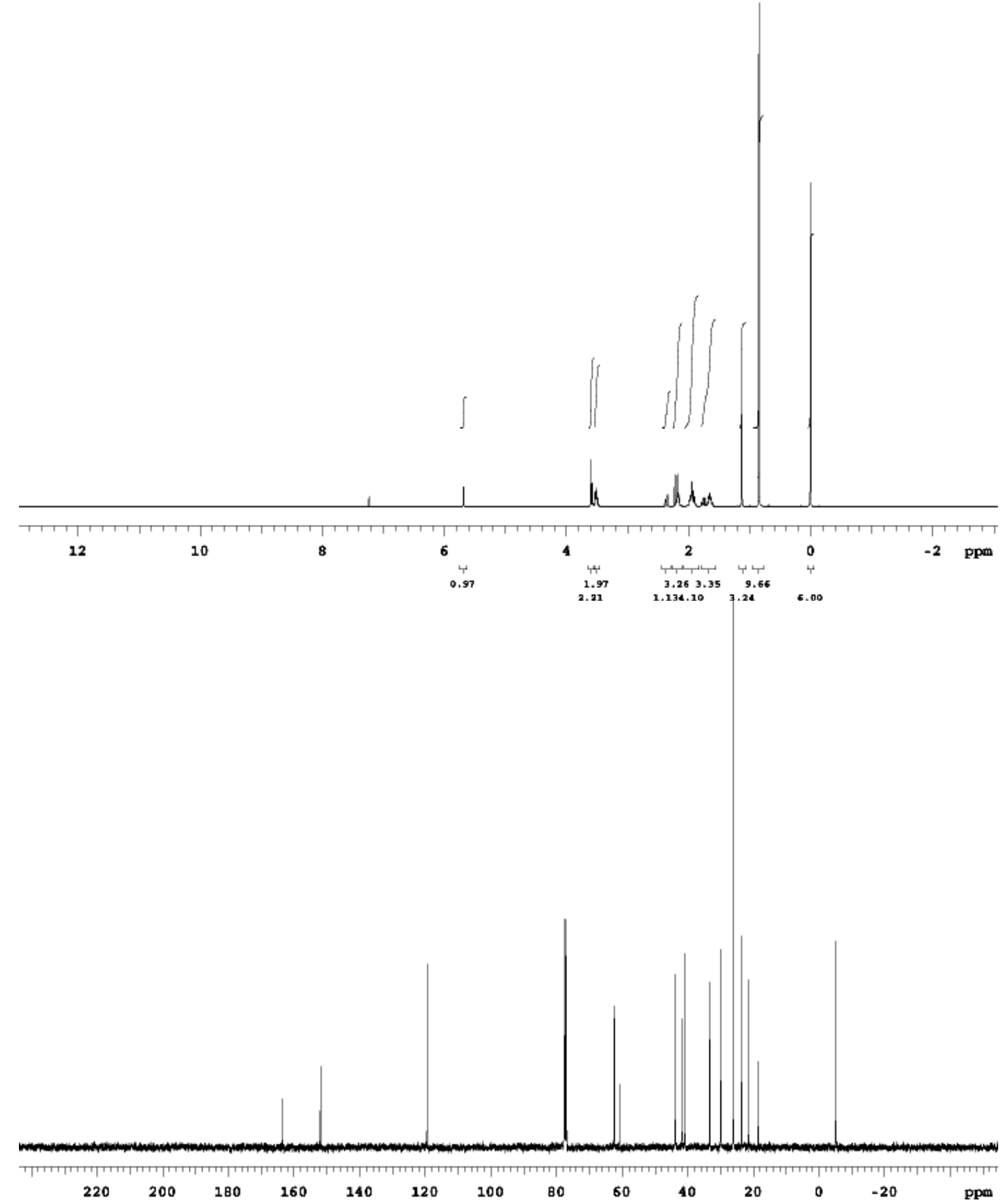

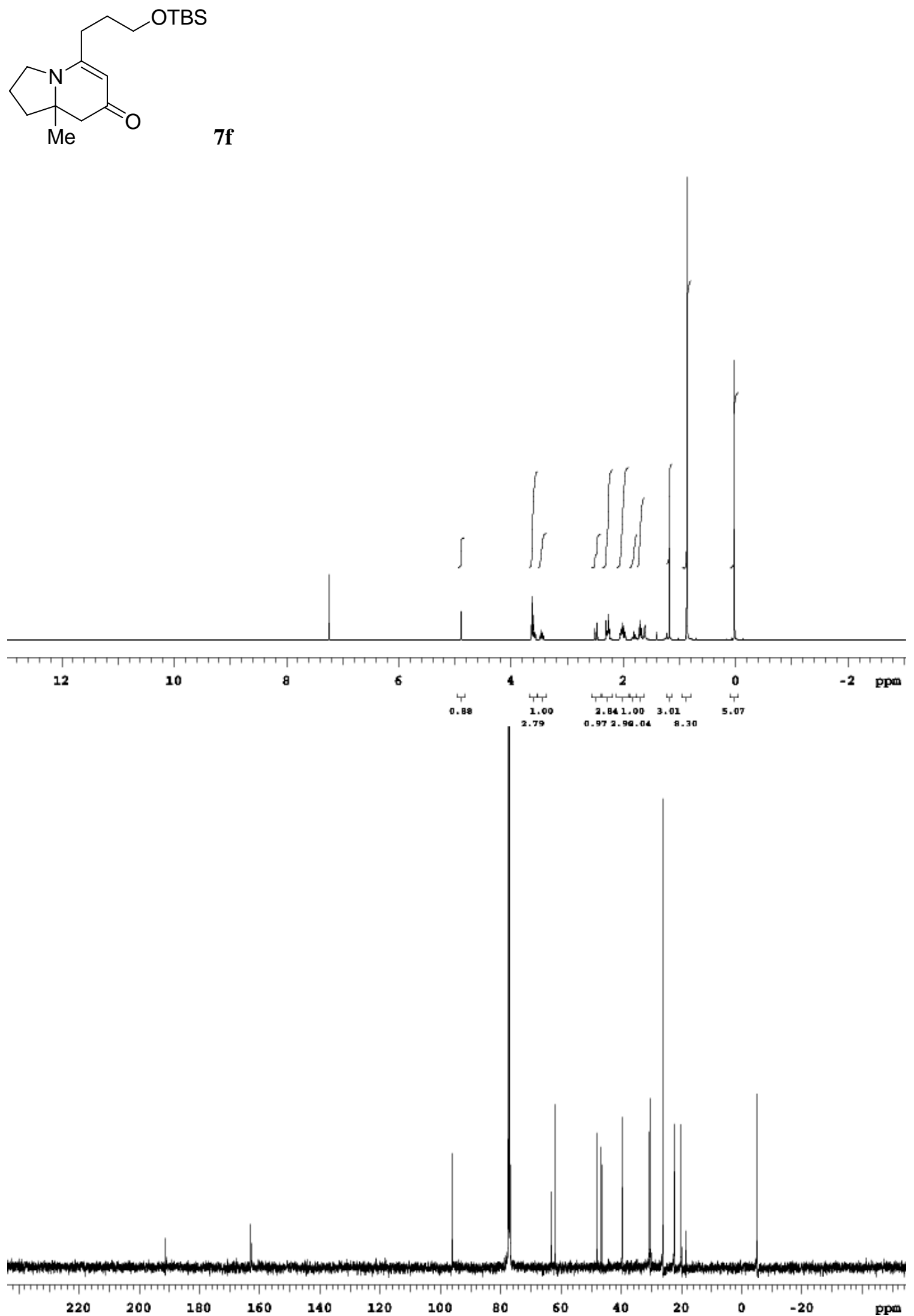
<smiles>CC12CCCN1C(=O)C=C(CC[OH+][SbH3])C2</smiles>
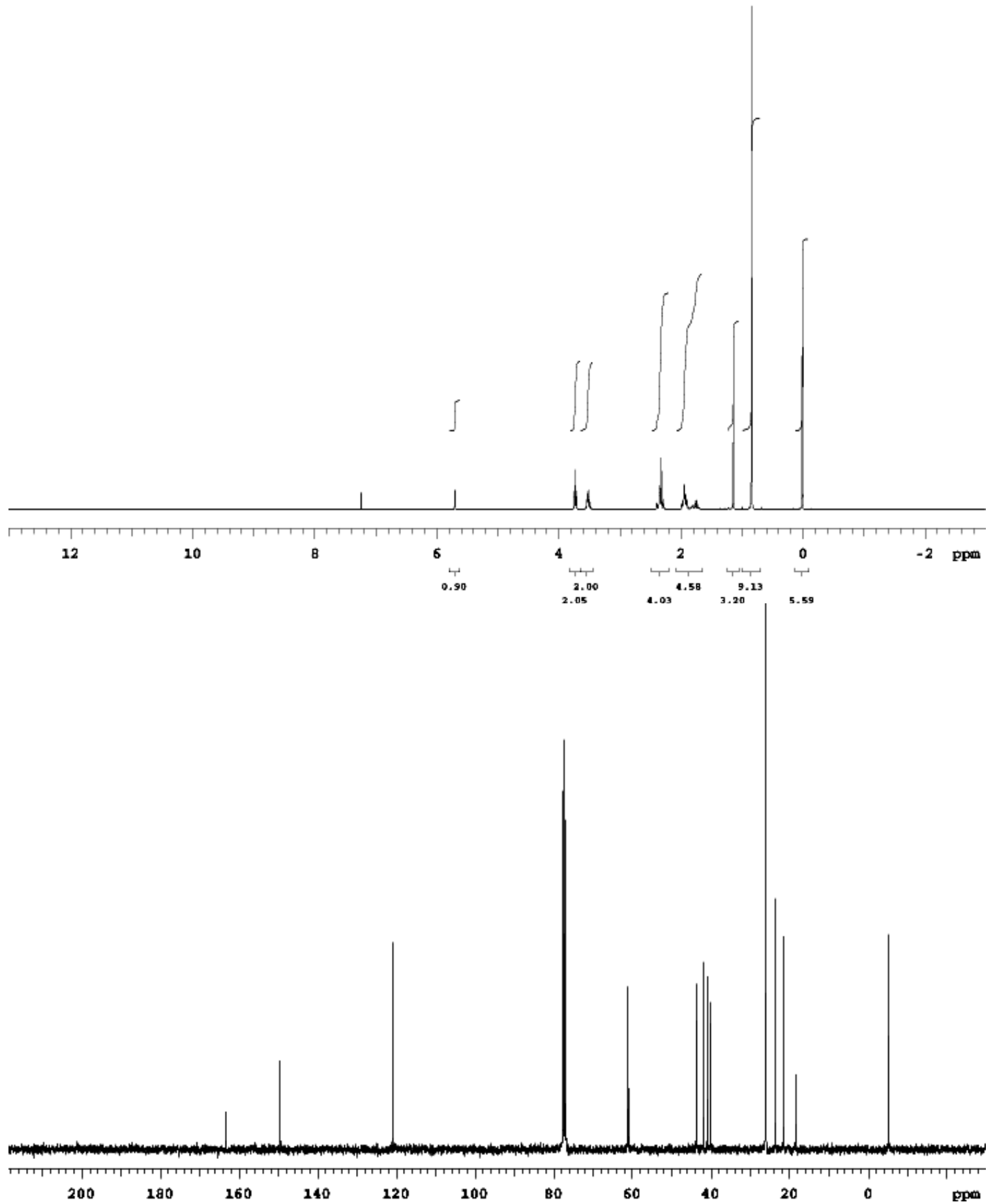
<smiles>CC(=O)CCCCC1=CC(=O)N2CCCC2(C)C1</smiles>

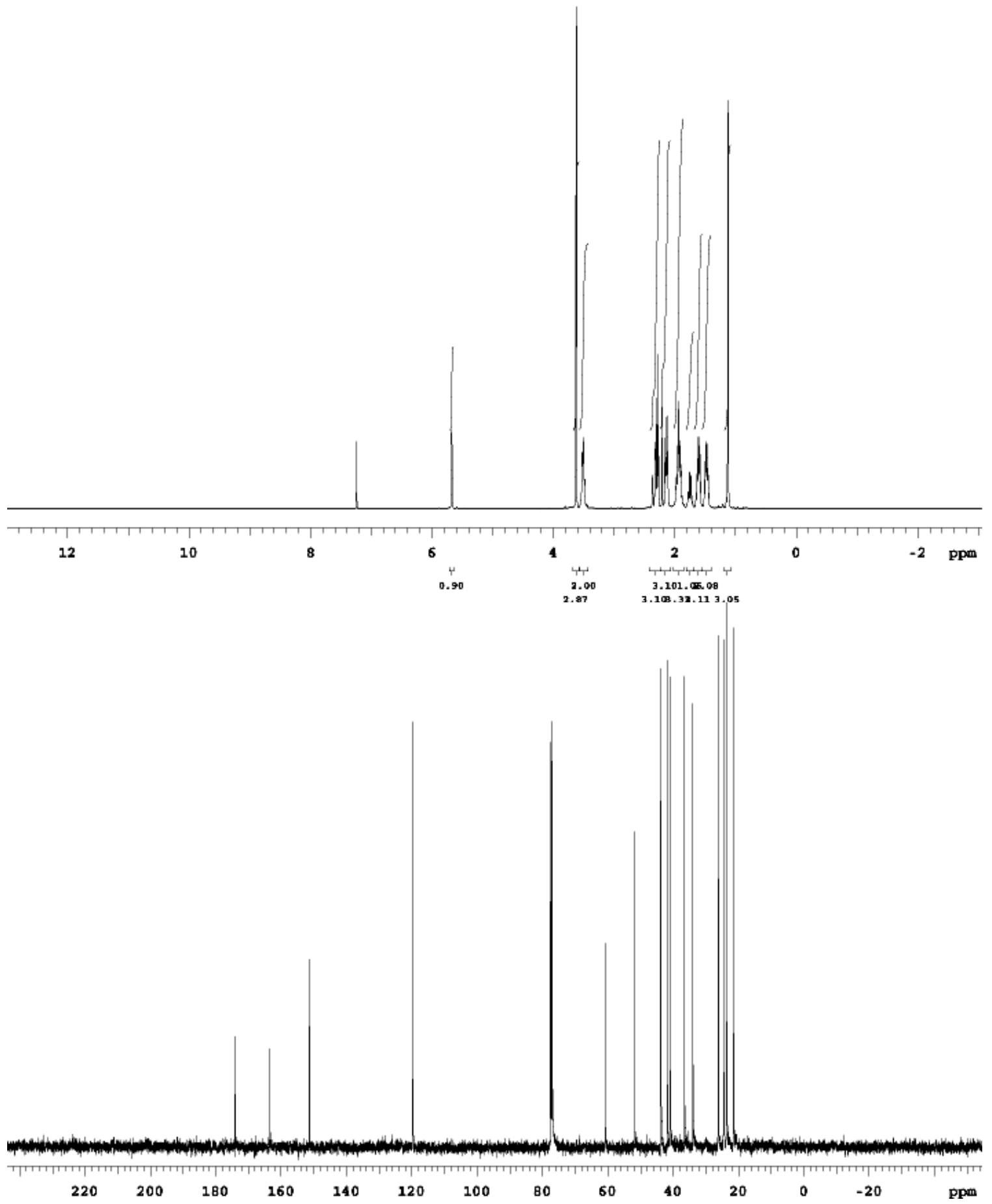



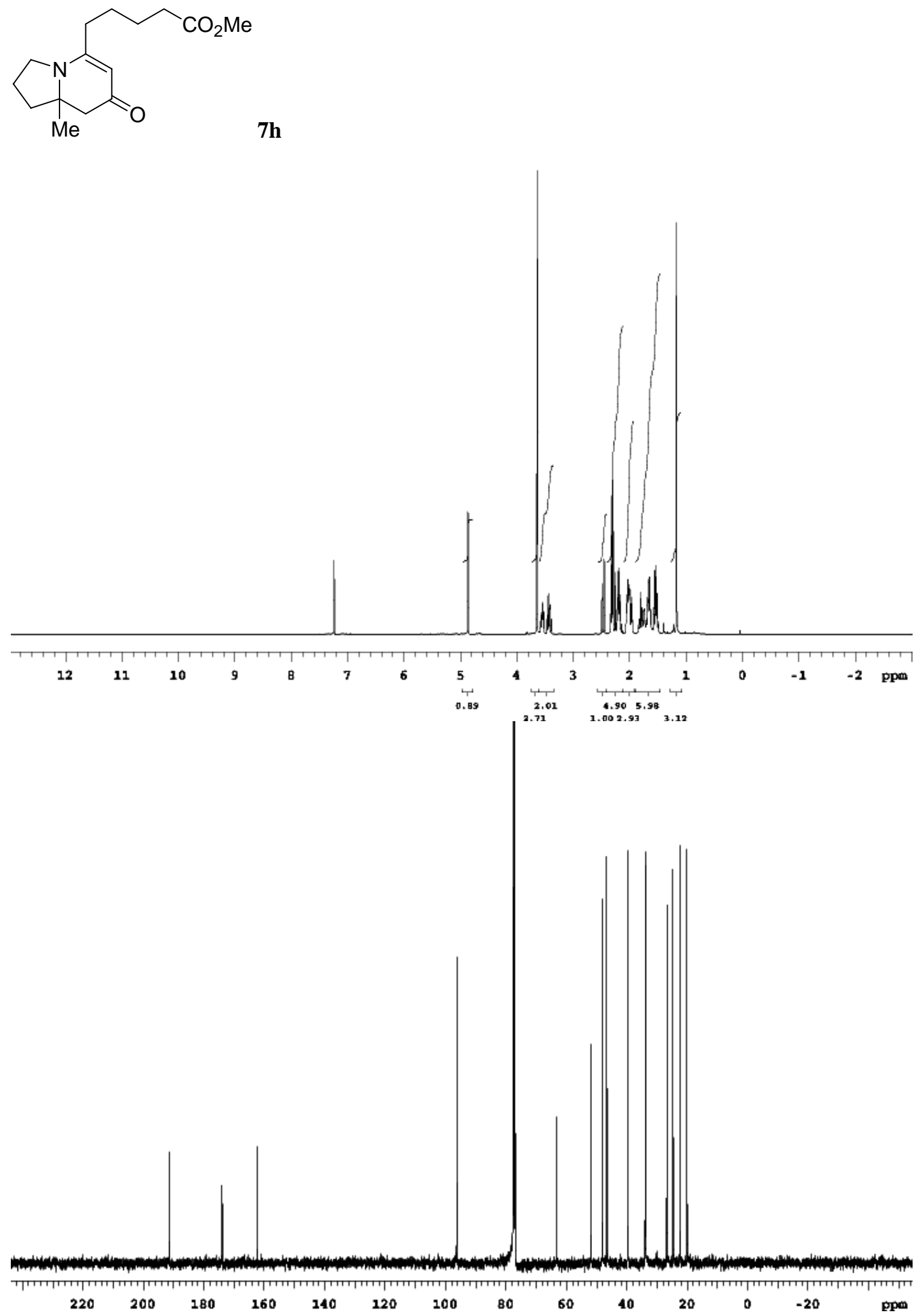
<smiles>CC12CCCN1C(=O)C=C(CCc1ccccc1)C2</smiles>
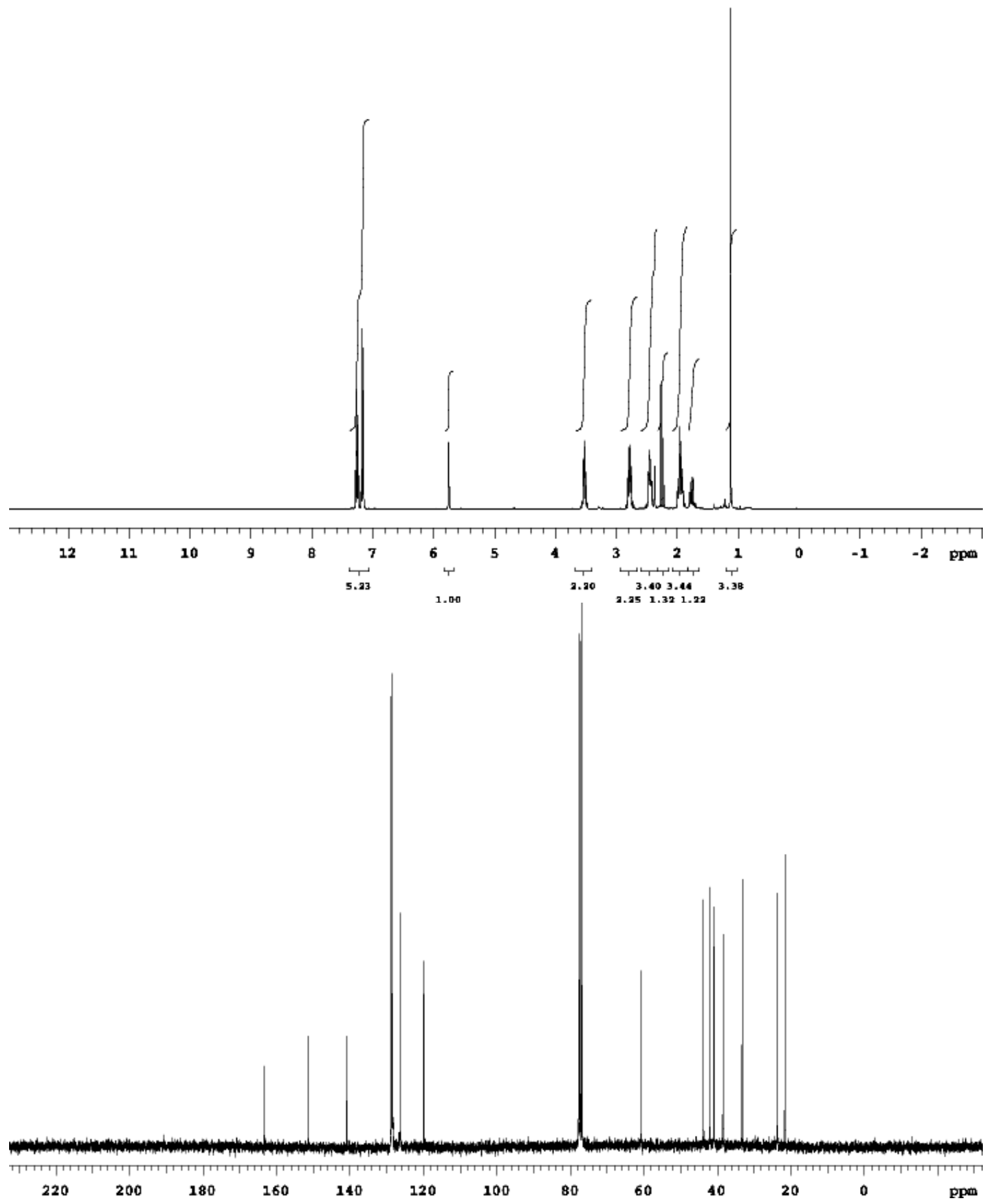
<smiles>CC12CCCN1C(=O)C=C(CCCCCl)C2</smiles>

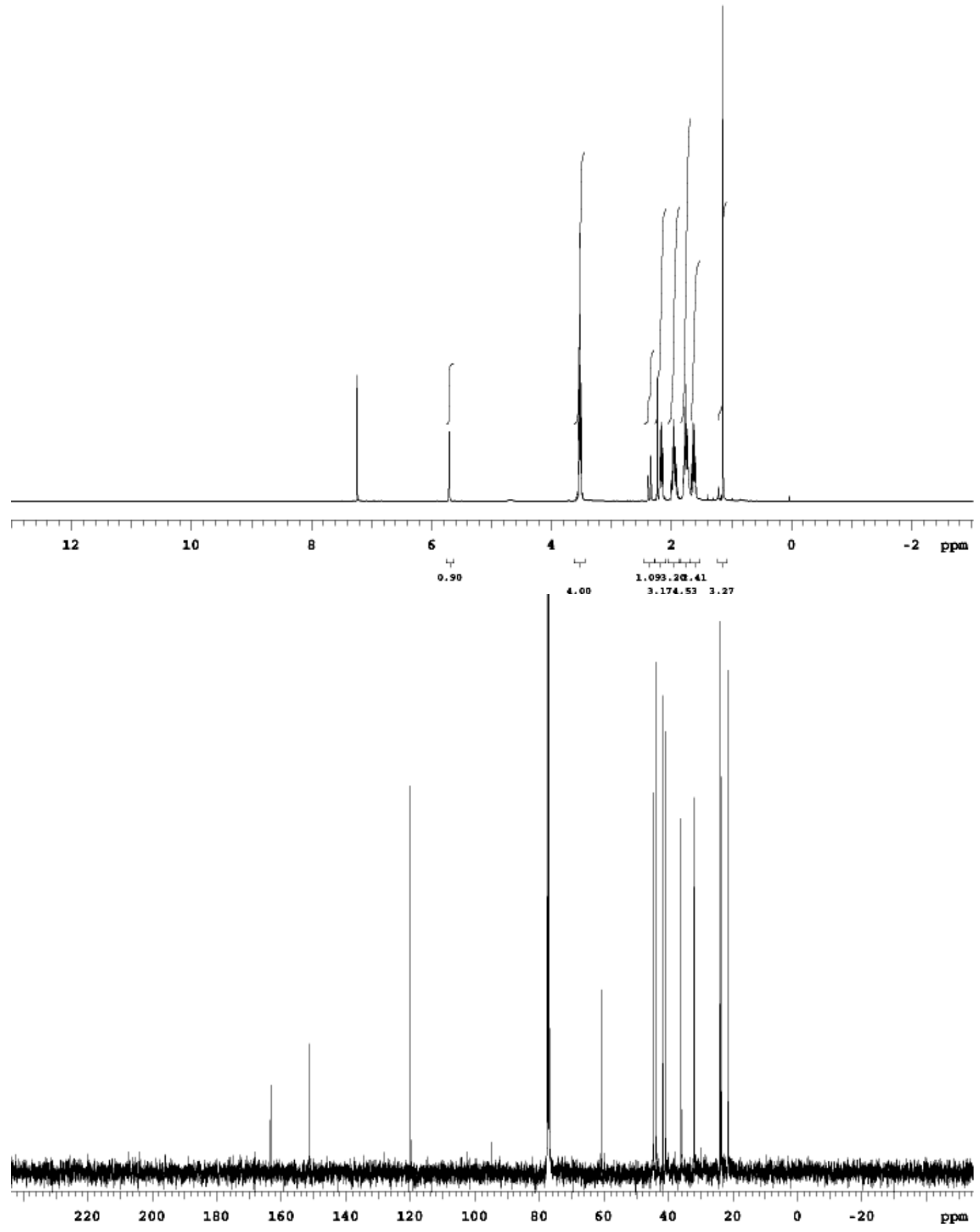




\section{I.12 Characterization of Pyridone Side Products.}<smiles>C=C(C)CCCn1c(-c2ccccc2)cc(-c2ccccc2)cc1=O</smiles>

1-(4-Methyl-pent-4-enyl)-4,6-diphenyl-1H-pyridin-2-one (8a): $\mathrm{R}_{f}=0.62$ (EtOAc(100\%)); IR (Thin Film) v 3059, 2935, 1654, 1602, 1537, $1490 \mathrm{~cm}^{-1} ;{ }^{1} \mathrm{H}$ NMR (400 MHz, $\left.\mathrm{CDCl}_{3}\right) \delta$ 7.60-7.55 $(2 \mathrm{H}, \mathrm{m}), 7.48-7.35(8 \mathrm{H}, \mathrm{m}), 6.79(1 \mathrm{H}, \mathrm{d}, J=2.0 \mathrm{~Hz}), 6.32(1 \mathrm{H}, \mathrm{d}, J=2.0 \mathrm{~Hz}), 4.55(1 \mathrm{H}, \mathrm{s}), 4.47$ $(1 \mathrm{H}, \mathrm{s}), 3.86(2 \mathrm{H}, \mathrm{m}), 1.85(2 \mathrm{H}, \mathrm{t}, J=7.0 \mathrm{~Hz}), 1.70(2 \mathrm{H}, \mathrm{tt}, J=7.0 \mathrm{~Hz}), 1.51(\mathrm{sH}, 3) ;{ }^{13} \mathrm{C}$ NMR $\left(100 \mathrm{MHz}, \mathrm{CDCl}_{3}\right) \delta 163.6,150.5,149.7,144.6,137.7,135.9,129.5,129.4,129.1,128.9,128.8$, 127.0, 115.9, 110.5, 107.8, 45.5, 35.1, 26.5, 22.2; MS (EI) m/e (rel intensity) 330 (100), 260 (19), 248 (40), 247 (24), 179 (19), 167 (14); HRMS (EI) m/e calcd $\left(\mathrm{M}^{+}\right)$330.1858, found 330.1853.<smiles>C=C(CCCn1c(CCCCCCC)cc(CCCCCC)cc1=O)C(C)C</smiles>

4,6-Bis-(4-methoxy-phenyl)-1-(5-methyl-4-methylene-hexyl)-1H-pyridin-2-one (10g): $\quad \mathrm{R}_{f}=$ 0.60 (EtOAc(100\%)); IR (Thin Film) v 2960, 1651, 1609, 1512, 1250, 1180, $1030 \mathrm{~cm}^{-1}$; ${ }^{1} \mathrm{H}$ NMR (400 MHz, CDCl $) \delta 7.51(2 \mathrm{H}, \mathrm{d}, J=9.0 \mathrm{~Hz}), 7.28(2 \mathrm{H}, \mathrm{d}, J=8.5 \mathrm{~Hz}), 6.96$ $(2 \mathrm{H}, \mathrm{d}, J=9.0 \mathrm{~Hz}), 6.91(2 \mathrm{H}, \mathrm{d}, J=9.0 \mathrm{~Hz}), 6.72(1 \mathrm{H}, \mathrm{d}, J=2.0 \mathrm{~Hz}), 6.27(1 \mathrm{H}, \mathrm{d}, J=2.0$ $\mathrm{Hz}), 4.59(1 \mathrm{H}, \mathrm{s}), 4.47(1 \mathrm{H}, \mathrm{s}), 3.89-3.83(2 \mathrm{H}, \mathrm{m}), 3.84(3 \mathrm{H}, \mathrm{s}), 3.80(3 \mathrm{H}, \mathrm{s}), 2.00(1 \mathrm{H}$, sept, $J=6.5 \mathrm{~Hz}), 1.87(2 \mathrm{H}, \mathrm{t}, J=7.5 \mathrm{~Hz}), 1.69(2 \mathrm{H}, \mathrm{tt}, J=7.5,7.5 \mathrm{~Hz}), 0.88(6 \mathrm{H}, \mathrm{d}, J=$ $7.0 \mathrm{~Hz}) ;{ }^{13} \mathrm{C}$ NMR $\left(100 \mathrm{MHz}, \mathrm{CDCl}_{3}\right) \delta 163.7,160.9,160.3,154.5,149.8,149.5,130.2$, 129.9, 128.3, 128.2, 114.5, 114.1, 107.8, 106.9, 55.6, 55.5, 45.6, 33.4, 31.8, 27.1, 21.9; HRMS (ESI) m/e calcd $\left(\mathrm{M}+\mathrm{H}^{+}\right)$418.2377, found 418.2379.<smiles>C=C(CCCn1c([C+](C)([O-])CCCCCC)cc(C(C)(C)C)cc1=O)C1CCCCC1</smiles>

1-(4-Cyclohexyl-pent-4-enyl)-4,6-bis-(4-methoxy-phenyl)-1H-pyridin-2-one (10h): $\mathrm{R}_{f}=0.60$ $(\operatorname{EtOAc}(100 \%))$; IR (Thin Film) v 2926, 2850, 1651, 1609, 1509, 1250, 1179, 1031, $824 \mathrm{~cm}^{-1} ;{ }^{1} \mathrm{H}$ NMR (400 MHz, CDCl $)_{3} \delta 7.51(2 \mathrm{H}, \mathrm{d}, J=9.0 \mathrm{~Hz}), 7.28(2 \mathrm{H}, \mathrm{d}, J=8.5 \mathrm{~Hz}), 6.95(2 \mathrm{H}, \mathrm{d}, J=9.0$ $\mathrm{Hz}), 6.91(2 \mathrm{H}, \mathrm{d}, J=8.5 \mathrm{~Hz}), 6.73(1 \mathrm{H}, \mathrm{d}, J=1.5 \mathrm{~Hz}), 6.27(1 \mathrm{H}, \mathrm{d}, J=1.5 \mathrm{~Hz}), 4.57(1 \mathrm{H}, \mathrm{s}), 4.48$ $(1 \mathrm{H}, \mathrm{s}), 3.89-3.84(2 \mathrm{H}, \mathrm{m}), 3.85(3 \mathrm{H}, \mathrm{s}), 3.81(3 \mathrm{H}, \mathrm{s}), 1.87$ (2H, t, $J=7.5 \mathrm{~Hz}), 1.71-1.56(8 \mathrm{H}, \mathrm{m})$, 1.23-0.96 (5H, m); ${ }^{13} \mathrm{C}$ NMR (100 MHz, $\left.\mathrm{CDCl}_{3}\right) \delta 163.8,160.9,160.3,153.9,149.8,149.4$, 130.2, 129.9, 128.4, 128.2, 114.5, 114.1, 107.8, 107.4, 55.6, 45.6, 43.8, 32.5, 32.4, 27.3, 26.9, 26.6; HRMS (ESI) $\mathrm{m} / e$ calcd $\left(\mathrm{M}+\mathrm{H}^{+}\right)$458.2690, found 458.2667 . 


\section{I.13 Determination of Pyridone Isomer.}

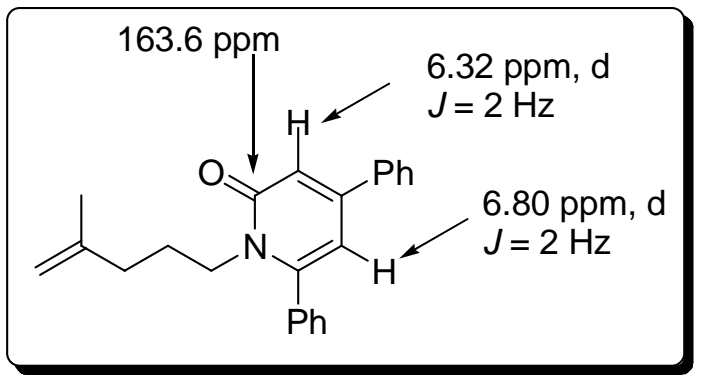

4-Pyridones
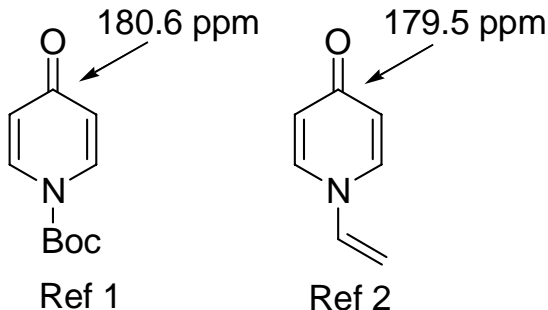

Ref 2

Matching isomer

$163.5 \mathrm{ppm}$<smiles>CCc1c(-c2ccccc2)c(-c2ccccc2)[nH]c(=O)c1CC</smiles>

Ref 3
Non-matching isomers<smiles></smiles>

Ref 4
$163.4 \mathrm{ppm}$

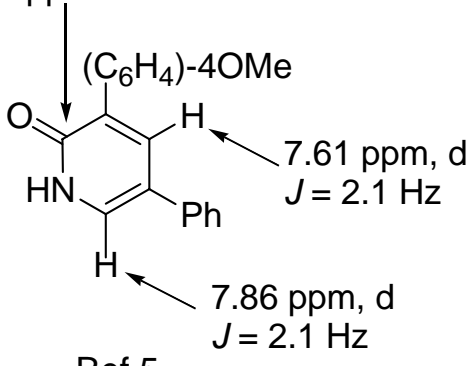

References

1. Lim, S. H.; Curtis, M. D.; Beak, P. Org. Lett. 2001, 3, 711-714.

2. Blouin, M.; Frenette, R. J. Org. Chem. 2001, 66, 9043-9045.

3. Carles, L.; Narkunam, K.; Penlou, S.; Rousset, L.; Bouchu, D.; Ciufolini, M. A. J. Org. Chem. 2002, 67, 4304-4308.

4. Yamamoto, K.; Yamazaki, S.; Murata, I. J. Org. Chem. 1987, 52, 5239-5243.

5. Sutherland, A.; Gallagher, T. J. Org. Chem. 2003, 68, 3352-3355. 


\section{I.14 Pyridone Byproduct ${ }^{1} \mathrm{H}$ and ${ }^{13} \mathrm{C}$ NMR Spectra}<smiles>C=C(C)CCCn1c(-c2ccccc2)cc(-c2ccccc2)cc1=O</smiles>

$10 a$

-1_1_100_1

solvent, CDC1s

knblent temperfetar.

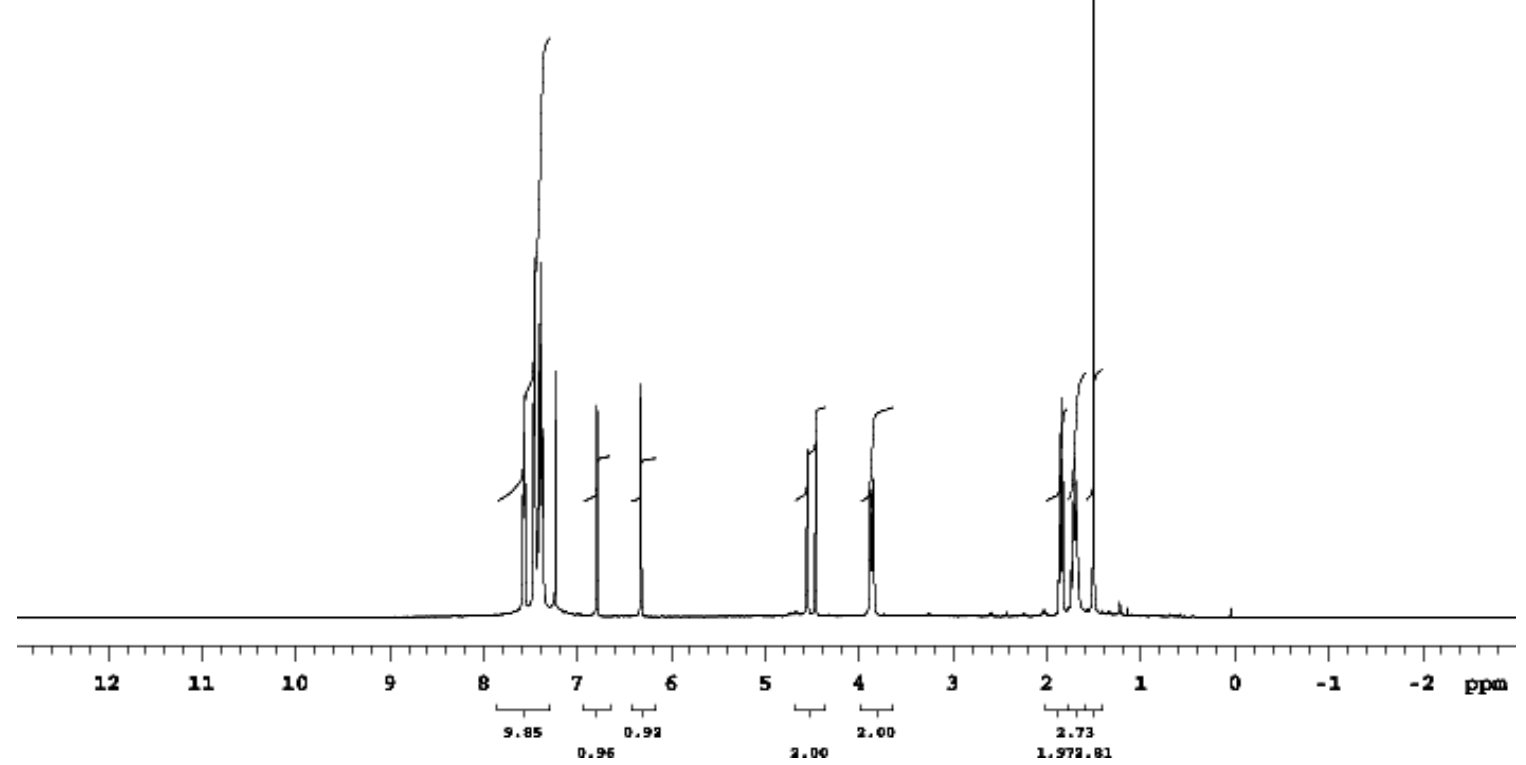

19e obsyrve

Solvent, CDC13

rablent tompaterate
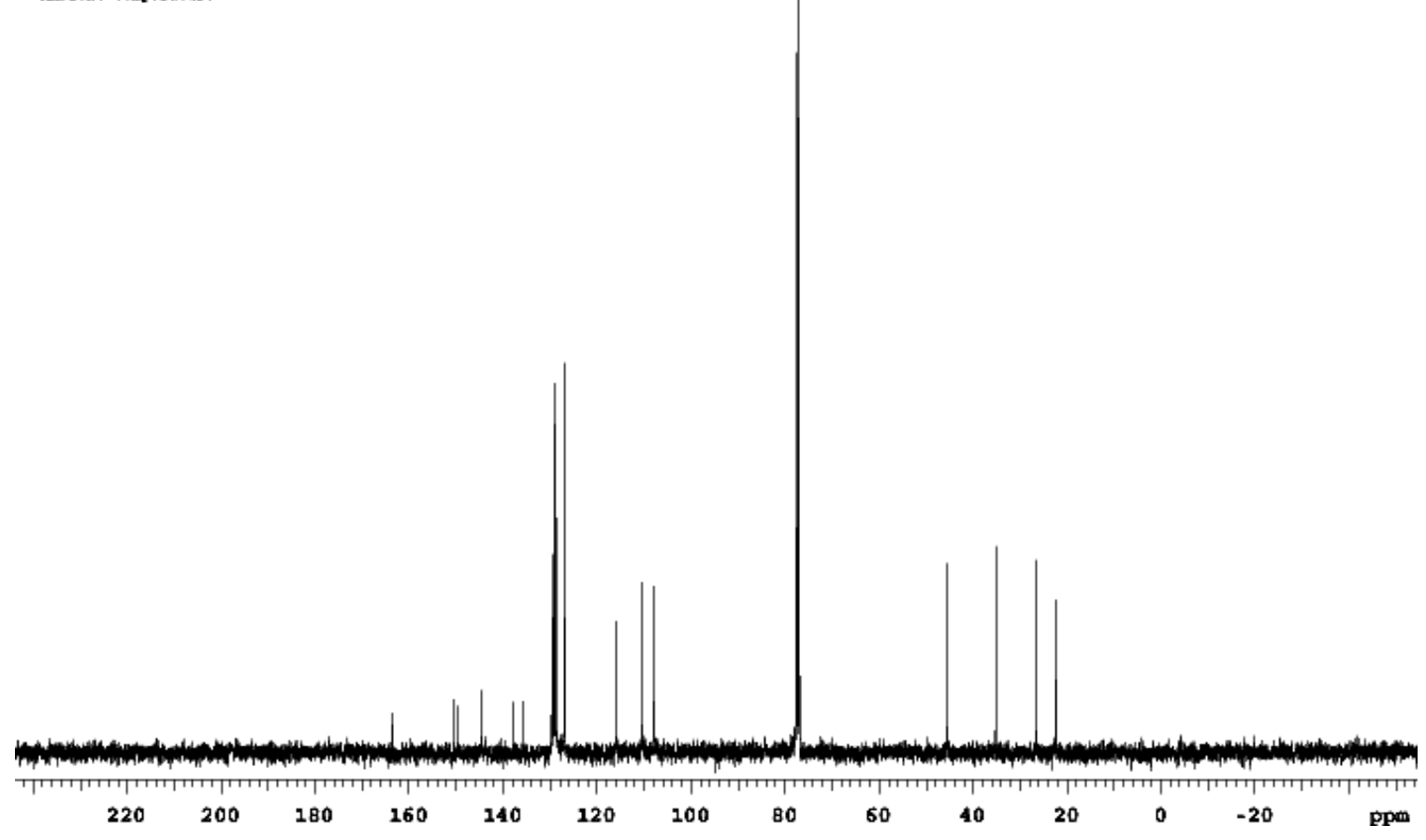

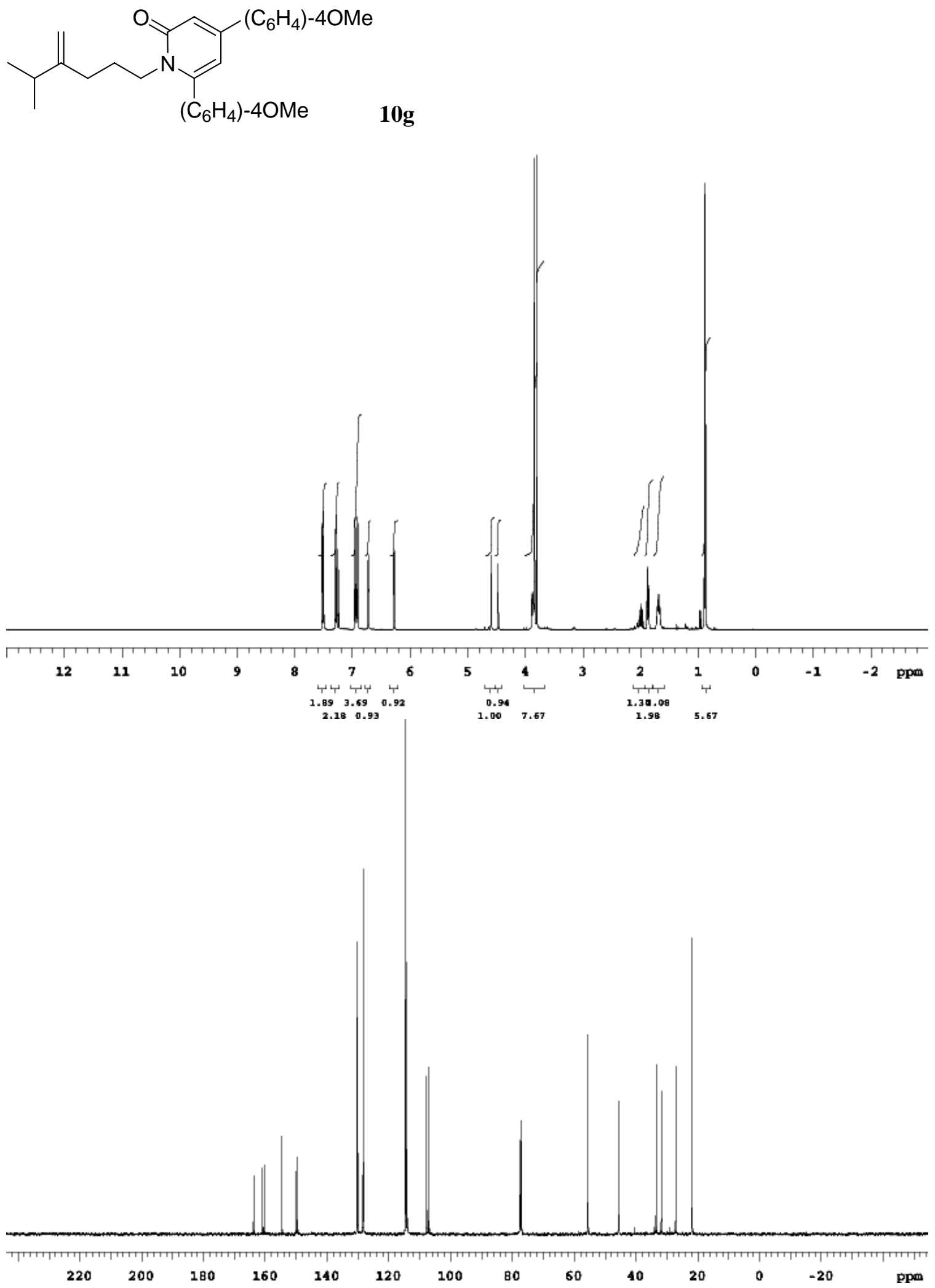
<smiles>C=C(CCCn1c(-c2ccccc2)cc(C(=O)OC)cc1=O)C1CCCCC1</smiles>
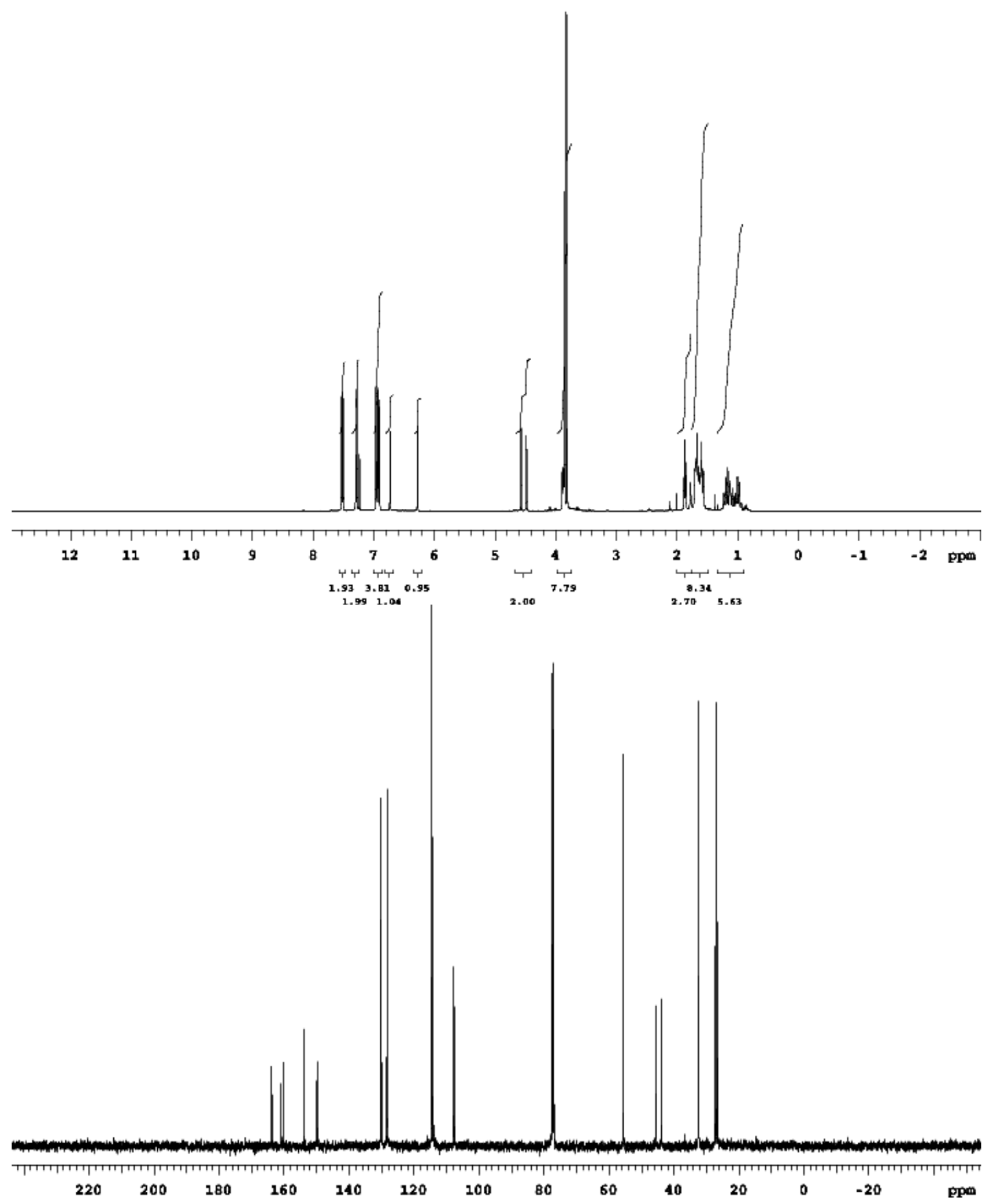